DE89 015229

\title{
Heat Capacity Measurements of Atoms arid Molecules Adsorbed on Evaporated Metal Films
}

\author{
Thomas William Kenny \\ (Ph.D. Thesis) \\ Materials and Chemical Sciences Division \\ Lawrence Berkeley Laboratory \\ 1 Cyclotron Road \\ Berkeley, California 94720
}

May 1989

\section{DISCLAIMER}

This report was prepared as an account of work sponsored by an agency of the United States Government. Neither the United States Government nor any agency thereof, nor any of their employees, makes any warranty, express or implied, or assumes any legal liability or responsibility for the accuracy, completeness, or usefulness of any information, apparatus, product, or process disclosed, or represents that its use would not infringe privately owned rights. Reference herein to any specific commercial product, process, or service by trade name, trademark, manufacturer, or otherwise does not necessarily constitute or imply its endorsement, recommendation, or favoring by the United States Government or any agency thereof. The views and opinions of authors expressed herein do not necessarily state or reflect those of the United States Government or any agency thereof. 


\title{
Heat Capacity Measurements of Atoms and Molecules
}

\section{Adsorbed on Evaporated Metal Films}

\author{
by
}

\section{Thomas William Kenny}

\begin{abstract}
Investigations of the properties of adsorbed monolayers have received great experimental and theoretical attention recently, both because of the importance of surface processes in practical applications such as catalysis, and the importance of such systems to the understanding of the fundamentals of thermodynamics in two dimensions. We have adapted the composite bolometer technology to the construction of microcalorimeters. For these calorimeters, the adsorprion substrate is an evaporated film deposited on one surface of an optically polished sapphire wafer. This approach has allowed us to make the first measurements of the heat capacity of submonolayer films of ${ }^{4} \mathrm{He}$ adsorbed on metallic films. In contrast to measurements of ${ }^{4} \mathrm{He}$ adsorbed on all other insulating substrates, we have shown that ${ }^{4} \mathrm{He}$ on silver films occupies a two-dimensional gas phase over a broad range of coverages and temperatures. Our apparatus has been used to study the heat capacity of Indium flakes, $\mathrm{CO}$ multilayers, ${ }^{4} \mathrm{He}$ adsorbed on sapphire and on $\mathrm{Ag}$ films and $\mathrm{H}_{2}$ adsorbed on Ag films. The results are compared with appropriate theories.
\end{abstract}

The Ge:Ga thermometers that were used in the construction of our calorimeters exhibited several interesting properties of their own. An investigation of bias induced nonlinearities in these thermometers confirmed some of the theoretical predictions for conduction dominated by variable range hopping. These results are discussed in Appendix I. An earlier project to produce, detect, and characterize the first continuously tunable farinfrared picosecond pulses is discussed in Appendix II. 


\section{Table of Contents}

Acknowledgement ................................................................. vi

Overview and motivation for heat capacity experiments............................. 2

Review of previous measurements.........................................2

${ }^{4} \mathrm{He}$ on Graphite..................................................... 3

${ }^{3} \mathrm{He}$ on graphite................................................... 7

Other noble gases...................................................... 7

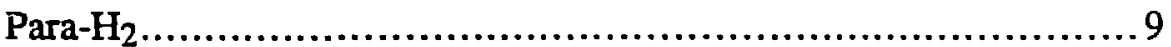

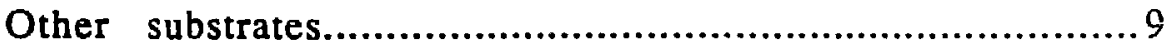

Motivation for this work.......................................................... 10

Metallic substrates..................................................... 11

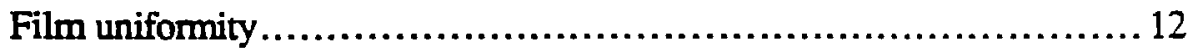

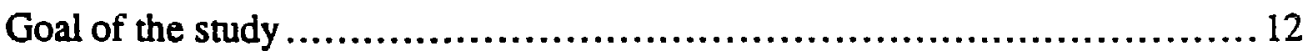

Signal/noise ratio for ideal ac calorimeters ..................................... 17

Experimental Apparatus .......................................................... 23

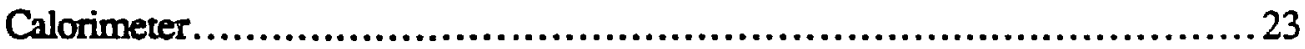

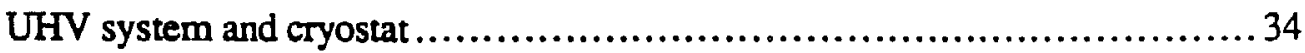

Measurement electronics............................................................36

Constraints of the method ......................................................... 40

Measurements.................................................................... 42

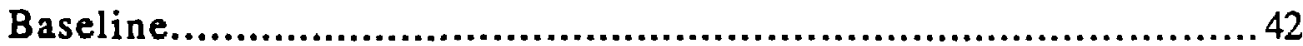

Indium samples.......................................................... 42

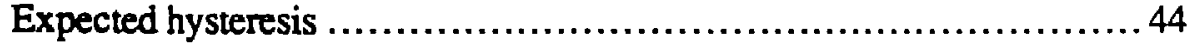

Experimental results................................................. 45

He adsorbed on sapphire .............................................. 48

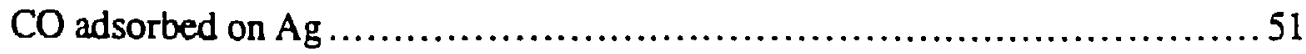

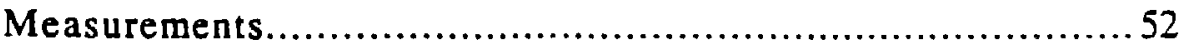




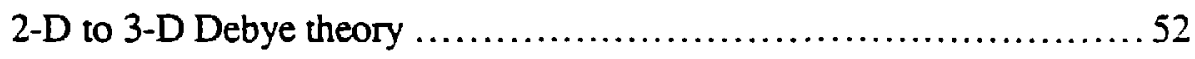

More complicated models ................................... 58

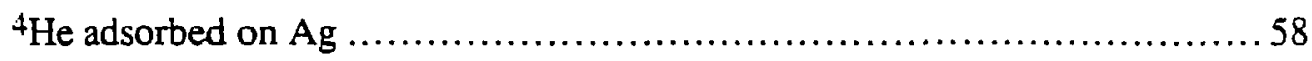

Heat Capacity Measurements. .................................... 59

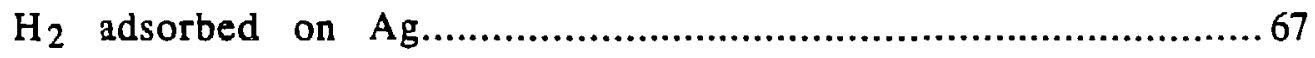

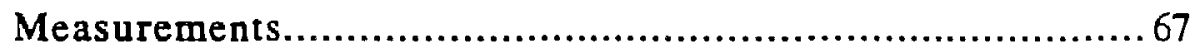

Future measurements ......................................................... 71

Possibilities at lower temperatures ..................................... 71

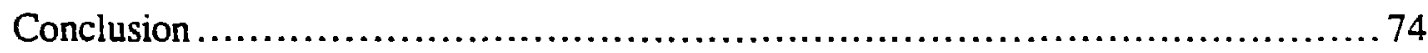

Appendix I Bias induced nonlinearities in neutron transmutation doped $\mathrm{Ge}$ at ${ }^{4} \mathrm{He}$

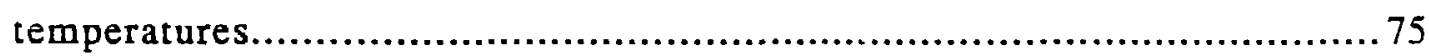

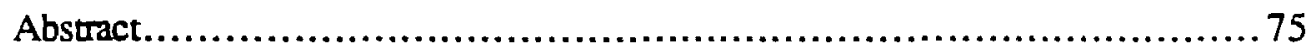

Introduction ............................................................... 75

Experimental Technique .............................................. 77

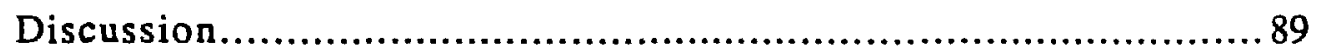

Conclusion ................................................................ 93

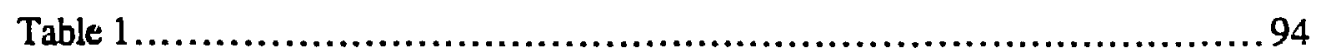

Appendix II Generation of intense tunable picosecond pulses in the far-infrared ........98

Abstract..................................................................... 98

Introduction ............................................................. 98

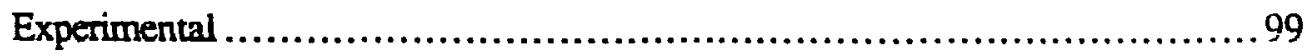

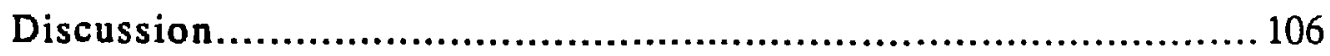


Table of Figures

Figure Number

Description

Fage Number

1

2

3

4

5

6

7

8

9
He on graphite phase diagram 4

Ag films viewed by STM

Diagram of calorimeter

24

Thermometer $R(T)$ measurements

27

Calorimeter rolloff neasurement

29

Bolometer response to picosecond pulse

30

Calorimeter fram $\varepsilon$ and mount

UHV system and cryostat

Measurement electronics

37

Heat capacity of calorimeter

In sample with hysteresis

In sample without hysteresis

Heat capacity of ${ }^{4} \mathrm{He}$ on sapphire

50

$\mathrm{CO}$ on Ag exponent vs coverage

Phonon modes for thin multilayer system

Multilayer density of states

Heat capacity of ${ }^{4} \mathrm{He}$ on $\mathrm{Ag}$ and 2-D Bose Gas

60

Sticking measurement

65

Heat capacity of $\mathrm{H}_{2}$ on $\mathrm{Ag}$

68

Test apparatus for thermometer characterization

Measured R(T)

83

I-V characteristics for heat-sunk thermometers

83

I-V curves plotted as $\log (R)$ vs $E / T$ 


\section{Acknowledgement}

Many people deserve mention for their contributions to my understanding of Physics, and to the maintenance of my mental condition through this long program. My apologies to all those not mentioned.

I first would like to thank all past and present members of the Polyps, an Intramural Frisbee team that we founded, and achieved great success with. The many victories, especially the B-league championship, provided much excitement and fun and will be remembered for many days to come. Among many, Peter Moore deserves special mention for encouraging me to bring the Polyps along to higher and higher levels of competition. As the Polyps merged with the Collegiate ream at Berkeley, Tim Pfafman was instrumental in encouraging us to participate. He deserves much of the credit for transforming the disorganized frisbee club into a team with national championship aspirations.

The participants in the weekly poker game also should be mentioned, for providing an occasional and inexpensive diversion, as well as some valuable experience in berting and bluffing, both of which are also valuable in everyday. Dan Marcus, who is a fan of bluffing, bad literature (often featuring Nazis or vampires, and occasionally both), extremely bad movies (Crazy Fat Ethel, for instance), and eventually a business partner, confidant, and Diet Coke drinking partner, deserves much credit for helping me dissipate my energies in only marginally self-destructive ways. Dan's only true enemies are the Japanese, who will continue to produce and market consumer electronics faster than he can afford to purchase them, even at his present salary.

In the research group, Gary Bernstein, Marc Fischer, Andrew Lange, and others in the astrophysics business were constant reminders that, no matter how badly things went, at least we could run our experiments again tomorrow. Bob Phelps deserves mention for 
his ability to balance the diverse demands of the physics and ballroom-dancing communities, and David Miller for his never-ending questions, and never-ceasing energy. I suppose we should all thank Dave's mom for making him what he is today, since no one else would want the credit (or blame). Carl Mears and Simon Verghese, of the new generation of Polyps, certainly provided their share of amlisement. Qing Hu, cursed with a loyalty to the Boston Celtics, is to be commended for enduring the Decade of the Lakers with dignity, humility, and much pain. Qing, it is never too late to become a Lakers fan.

Roger Tobin's guidance through the early years of my research was invaluable. His organization, his pursuit of meaningful results on a daily basis, and his experimental skills provided a regular example of reason applied to research. and he has influenced me greatly. In addition, his competitive nature, as demonsurated by his softball pitching, was proof to me that he could have been a marvelous Polyp, if the timing had been different.

Paul Richards deserves thanks for focusing the remainder of my energies onto a series of challenging and rewarding projects, the results of which are this thesis. I sincerely hope that the results were worth the patience that was required to deal both with my personality, and my tendency to devote much energy to the extra-curriculars. I often wonder how many man-hours of research were lost because of the group-wide participation in the Polyps.

Bruce Bauer, my high school physics teacher also deserves mention. I received 3 years of excellent instruction in the fundamentals of physics, and obtained much of my intuitive grasp of the subject. Without this head start, I highly doubt that I would be where I am today. 
My Father and Mother, and my brothers provided financial and emotional support many times throughiout this long process. There were some hard times and mistakes were made, but the support and care remained through to the end, and that's what really counts.

Kristi Rusch, temporarily my wife, and always a true friend, has as much to do with my reaching the conclusion of this thesis as anyone else. When I was young, she provided the confidence in me that eventually lead me to consider myself capable of such an achievement. In many imponant ways, I grew up with her, and Ill always owe her for that support.

Debby Kramer, the first female Polyp, has greatly brightened my iast two years at Berkeley. Her enthusiasm, support, and patience with my often irregular work hours, alltoo-regular poker games, frisbee practices, and bad movie festivals, and inability to close cupboards, drawers, and closets was especially important to me. I hope I wasn't all that frustrating...

Finally, I thank AT\&T Bell Laboratories, for the fellowship support through the last 3 years, which removed some of the financial penalties associated with extended graduate research, and made life considerably more pleasant.

This work was supported in part by the Director, Office of Energy Research, Office of Basic Energy Sciences, Materials Sciences Division of the U. S. Department of Energy under Contract No. DE-AC03-76SF00098. 
Heat Capacity Measurements of Atoms and Molecules Adsorbed on Evaporated Metal Films

By

Thomas William Kenny

B. Physics (University of Minnesota) 1983

M.A. (University of California) 1988

\section{DISSERTATION}

Submitted in partial satisfaction of the requirements for the degree of DOCTOR OF PHILOSOPHY

in

PHYSICS

in the

GRADUATE DIVISION

of the

UNIVERSITY OF CALIFORNIA at BERKELEY

Materials and Chemical Sciences Division

Lawrence Berkeley Laboratory

1 Cyclotron Road

Berkeley, CA 94720 


\section{Qverview and motivation for heat capacity experiments}

Surfaces play an important role in many processes of great technological importance. Yat, the understanding of most phenomena that occur at surfaces is incomplete, even at the most fundamental level. Clearly, the development of a complete understanding of these phenomena would directly lead to improvements in their applications. For this reason, the study of phenomena at surfaces is currently a very active field.

Recent experimental investigations of the properties of atoms and molecules adsorbed on solid surfaces have produced many interesting results. The physisorbed noble gases are the systems which involve the least complicated interactions. Because of the spherical symmetry of the adsorbate and the simplicity of the physisorption interaction, complete theoretical descriptions can be formulated and compared with experimental results. 1

Experimental measurements of the heat capacity of adsorbates have provided much of the thermodynamic information needed for the identification of phases and the transitions between them. ${ }^{2}$ The subject of this thesis is the extension of such measurements to an entirely new collection of substrates. In order to fully motivate this development, it is necessary to review the work by others in this field.

\section{Review of previous measurements}

The earliest measurements of the heat capaciry of He monolayers were carried out on substrates of sintered copper sponge plated with Argon. ${ }^{3}$ The complicated structure of the Cu substrate provided by this material is smoothed somewhat by the Ar plating. The measurements showed the existence of a two-dimensional solid below $3 \mathrm{~K}$, and broad 
maxima in the heat capacity above $3 \mathrm{~K}$. These maxima were taken to indicate the occurrance of a first order phase transition in the He overlayer.

\section{He on Graphite}

Exfoliated graphite was found to provide large amounts of surface area per unit volume which is composed mostly of basal-plane surfaces. It was anticipated that the density of carbon atoms in the basal plane was high enough that low-density physisorbed overlayers would experience only slight lateral variations in the binding energy. ${ }^{2}$ A series of early calculations of the He-graphite interaction supported this assertion. ${ }^{4}$ For these reasons, exfoliated graphite was expected to provide a nearly ideal substrate for thermodynamic measurements of physisorbed overlayers.

An extensive study was carried out of the heat capacity of ${ }^{3} \mathrm{He}$ and ${ }^{4} \mathrm{He}$ adsorbed on exfoliated graphite. 5 The phase diagram of the ${ }^{4} \mathrm{He}$ adsorbed on graphite system is shown in Fig. 1. In the following discussion, a monolayer is defined as the coverage at which an additional adsorbed atom would be promoted to the second layer, this coverage is approximately 0.12 atoms $\AA^{-2}$ for He adsorbed on graphite. At coverages greater than 0.08 atoms $\AA^{-2}$, the He forms a hexagonal two-dimensional solid which is incommensurate with the graphite substrate. This 'floating' solid then melts to an isotropic fluid at temperatures above $3 \mathrm{~K}$, depending on the coverage. The anomalies associated with these melting transitions are quite pronounced as shown in the inset to Fig. 1.

At coverages between 0.08 and 0.05 atoms $\AA^{-2}$, the He forms a $\sqrt{3} \times \sqrt{3}$ commensurate solid. This commensurate solid melts at about $3 \mathrm{~K}$, producing very large, sharp peaks in the measured heat capacity. 


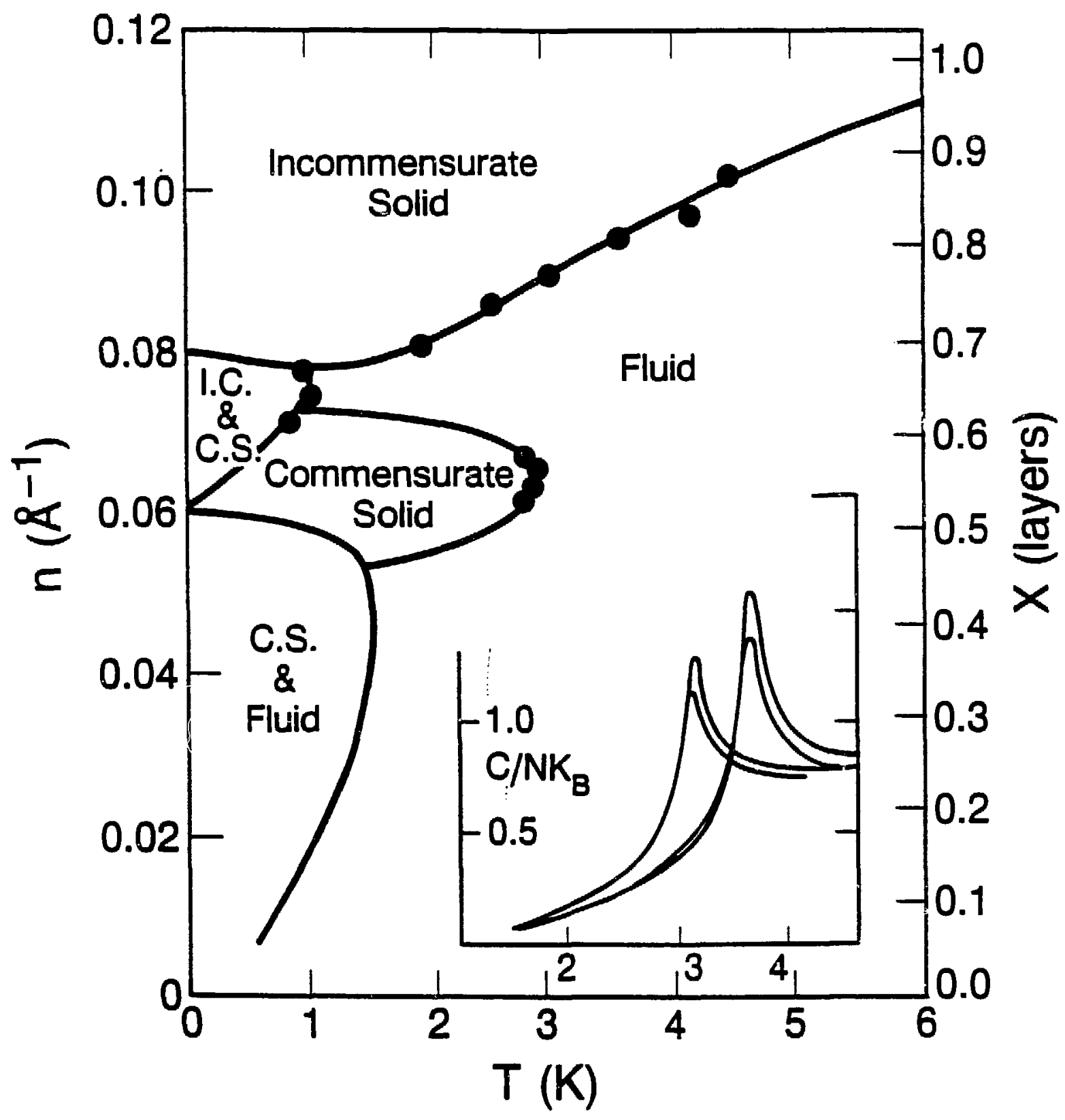

XEL 893-5065

Figure 1 2-dimensional phase diagram of He submonolayers physisorbed on graphite. Typical anomalies associated with the melting of the incommensurate solid are shown in the inset. Taken from Campbell and Bretz (1985). 
At coverages beiow 0.05 atoms $\AA^{-2}$, the ${ }^{4} \mathrm{He}$ forms an ideal ciassical twodimensional gas at temperatures above $2.5 \mathrm{~K}$. As temperatures are reduced, small, rounded bumps in the heat capaciry appear at about $1 \mathrm{~K}$. Below the bumps, the heat capacity falls to zero.

A theory of the melting of two-dimensional magnetic systems has been formulated by Kosterlitz and Thouless 6 and extended to the case of monolayer solids by Halperin, Nelson ${ }^{7}$ and Young 8 (KTHNY). This theory makes several specific predictions: The twodimensional solid melts by a two-stage process. In the first stage, bound dislocation pairs are thermally excited, and become unbound at a certain temperature. After the unbinding of the dislocations, the fluid has no long-range positional order, but retains long-range bondorientational order. This ordered fluid, known as the hexatic phase, gradually becomes isotropic through the unbinding of disclination pairs, which occurs at a higher temperature. Measurements of the temperature dependence of the heat capacity in such a system should reveal two separate continuous melting transitions.

Other mechanisms for the melting of the two-dimensional solid have been proposed. In particular, it has been suggested that the melting can occur due to the formation of grain boundaries between differently oriented solid crystallites. ${ }^{9}$ Since the free energy for the formation of such boundaries decreases with temperature, there should exist a temperature at which the boundaries become energetically favorable, leading to a first-order transition directly from a hexagonal solid to an isotropic fluid.

The melting of the high-density incommensurate He monolayers on graphite was expected to be a test case for melting in two-dimensions. A careful investigation of this transition was carried out, and the melting peaks were shown to be broadened by inhomogenities in the graphite substrate. ${ }^{10}$ An analysis of the inhomogenities was used to deconvolve the intrinsic melting peaks from the observed peaks. The intrinsic peaks were 
then compared with the predicted widths for KTHNY and grain boundary-mediated melting. On the basis of this comparison, the authors concluded that the peaks were too sharp to be of the KTHNY type, and that the melting process must be of first order. Subsequent calculations of KTHNY melting have shown that narrower peaks are possible, and that the identification of the melting transition on the basis of the heat capacity data is not possible. 11

The melting of the intermediate coverages has been shown to be consistent with theoretical calculations within three-state Potts models, in which melting occurs by motion of the atoms among the normally unoccupied sites in the $\sqrt{3} \times \sqrt{3}$ overlayer. 12

The low coverage data can be accurately fit at temperatures above $2 \mathrm{~K}$ by quantum mechanical second-virial coefficient corrections to the ideal gas law which are appropriate for bosons. ${ }^{13}$ Below $2 \mathrm{~K}$, these corrections predict continued upward deviations in the heat capacity, which are not consistent with the experimental results. Recent measurements of the binding energy for He on graphite as a function of position on the graphite surface have shown that the lateral variation, or "corrugation", in the binding energy is considerably larger than originally thought ${ }^{14}$ This comgation gives rise to a complicated single-particle dispersion relation for He moving across the surface of graphite. In particular, a large band gap appears at about $11 \mathrm{~K}$ in the density of states, and it is this gap which causes the observed bumps in the heat capacity. 15 Models which completely ignore adsorbate-adsorbate interactions in favor of adsorbate-substrate interactions have been used to calculate the heat capacity of $\mathrm{He}$ on bare graphite with good agreement up to coverages approaching $1 / 3$ of a monolayer. 
At very low coverage, the experimental results can be fit by models which assume a collection of independent harmonic oscillators. 5 The physical picture is one of He atoms oscillating within specific sites on the graphite substrate, perhaps at the grain boundaries.

\section{He on graphite}

Monolayers of ${ }^{3} \mathrm{He}$ exhibit the same behavior as monolayers of ${ }^{4} \mathrm{He}$ at coverages above 0.05 atoms $\AA^{-2}$ and temperatures above $0.5 \mathrm{~K}$. At coverages below 0.05 atoms $\AA^{-2}$ and termperatures below $1.5 \mathrm{~K}$, the measured temperature dependence of the heat capacity deviates downward from the ideal gas limit. These deviations are accurately fit at temperatures above $1 \mathrm{~K}$ by quantum mechanical second-virial coefficient corrections to the ideal gas law which are appropriate for fermions. At lower temperatures, magnetic ordering phenomena dominate the measured heat capacity.

\section{Qther noble gases}

The phase diagrams of the remaining noble gases adsorbed on graphite have also been thoroughly investigated. For the case of neon, a two-dimensional triple point is observed at $13.5 \mathrm{~K}$, indicating the presence of two-phase coexistance. ${ }^{16}$ For Ne on graphite, the two-dimensional solid is bound, leading to the formation of islands. This is in contrast to the case of He on graphite, for which the solid phases spread to fill the available area, and two-phase coexistance is not observed. At coverages above those of the triple point, the compressed incommensurate monolayers of Ne exhibit melting peaks similar to those of the incommensurate overlayers of $\mathrm{He}$.

Monolayers of Ar on graphite have been widely studied 17 Recent high-resolution heat capacity measurements at submonolayer coverage have shown a small, sharp peak, followed by a large, broad peak at higher temperature. ${ }^{18}$ These results have been interpreted as evidence for first-order, triple-point melting followed by a remnant of the 
hexatic-isotropic transition, or as consistent with the two-stage KTHNY melting. Since the KTHNY theory does not predict the widths of the melting peaks, the observed sharpness of the first peak is not necessarily evidence for a first-order transition. Synchrotron $\mathrm{x}$-ray experiments have shown evidence for continuous evolution of the correlation lengths in the vicinity of the peaks in the heat capacity, and are consistent with the predictions of KTHNY.19 At coverages near monolayer completion, the observed melting transition is consistent with the prediction for continuous melting transitions.

Monolayers of $\mathrm{Kr}$ form strongly bound commensurate solids whose melting transitions are well fit by lattice models. At coverages near monolayer completion, the melting transitions are very broad, and have not been fit satisfactorily by theory. ${ }^{20}$

Monolayers of Xe have received great attention. The Xe forms a triangular incommensurate solid on graphite over a broad range of coverages. Synchrotron X-ray scattering shows that the submonolayers melt by a first order process, but at higher coverages, the transition becomes continuous. ${ }^{21}$ Two consecutive peaks are observed in compressibility measurements of the continuous transition, and the results are consistent with the KTHNY theory. 22 The hexagonal order of the graphite substrate is assumed to contribute to the formation of the hexatic phase, but sophisticated analyses of the diffraction pattems indicate that the Xe-graphite hexagonal-ordering interaction is not strong enough to explain the results. Synchrotron $\mathrm{x}$-ray studies of Xe adsorbed on $\mathrm{Ag}(111)$, which has a considerably weaker substrate ordering field, have reproduced all the details of the Xe adsorbed on Graphite phase diagram, providing strong evidence for the intrinsic nature of the hexatic phase in Xe monolayers. ${ }^{23}$ However, $\approx$ zent heat capacity measurements of Xe adsorbed on graphite find evidence for only a single, first-order transition. ${ }^{24}$ 


\section{$\underline{\text { Para- }-\mathrm{H}_{2}}$}

Molecular hydrogen, because it is lighter and more polarizable, is an interesting adsorbate to compare with helium. ${ }^{25}$ The hydrogen molecule exists in two nuclear spin states. Para- $\mathrm{H}_{2}$ has an antisymmetric nuclear spin wave function and a symmetric sparial wave function, and ortho- $\mathrm{H}_{2}$ has the opposite. At room temperature, the population is evenly divided between the four states of the nuclear wave function, the anti-symmetric Pstate being a triplet, and the symmetric S-state being a singlet. When cooled and placed in the presence of inhomogeneous magnetic fields, such as near magnetic atoms, the ortho- $\mathrm{H}_{2}$ states decay to the para- $\mathrm{H}_{2}$ ground state. For studies of adsorbed monolayers, para- $\mathrm{H}_{2}$ has been most widely investigated because of its similarities and differences with ${ }^{4} \mathrm{He}$.

Monolayers of para- $\mathrm{H}_{2}$ can be prepared by mixing with paramagnetic salts prior to the adsorption on the substrate, or by allowing it to decay into the para state on the cold substrate. Investigations of the nuclear spin states after adsorption on graphite have shown that it instantaneously converts to para- $\mathrm{H}_{2}{ }^{26}$ Similar experiments on $\mathrm{Ag}$ substrates have given conflicting results. 27 The rapid conversion on some substrates may be due to the presence of magnetic impurities on the surface.

Submonolayers of para- $\mathrm{H}_{2}$ on graphite have been investigated with heat capacity measurements 28,29 and, more recently, with low energy electron diffraction (LEED). 30 The heat capacity measurements show a phase diagram similar to that of ${ }^{4} \mathrm{He}$, with the transitions shifted to $4 x$ higher temperatures because of the stronger adsorbate-adsorbate interactions.

\section{Qther substrates}

A number of other substrates have been used for thermodynamic investigations of physisorbed monolayers. Magnesium oxide smoke, which is prepared by burning 
magnesium in air, rapidly sealing the ashes in a canister, and baking under vacuum at 900 C, has uniform surfaces with square symmetry. The binding ene-gy for ${ }^{4} \mathrm{He}$ on $\mathrm{MgO}$ is more comugated than graphite, driving the formation of rectangular commensurate solids. Mylar, Mica, and other layered materials have been used, but difficulties in preparation and characterization of clean, uniform surfaces has been difficult.

Some variability in the characteristics of graphite can be obtained by plating with one or more solid monolayers of a heavy noble gas prior to He adsorption. ${ }^{31}$ The heavy noble gas forms an inert, incommensurate solid that partially smooths the variations in the He-graphite interaction. A study of the effects of plating on the behavior of ${ }^{4} \mathrm{He}$ submonolayers showed evidence for reductions in the corrugation of the binding energy. 32 Additional evidence for nucleation of solids near defects in the plating layer complicates the interpretation of these results.

\section{Motivation for this work}

As described above, the characteristics of the substrate and the interaction with the adsorbate often have important effects on the behavior of the adsorbate. In many cases, defects, impurities, or grain boundaries were responsible for broadened phase transitions. The corrugation in the adsorbate-substrate binding energy drives the formation of commensurate solids, introduces band-structure effects, and greatly increases the theoretical sophistication required to model the experimental results. The relatively small number of substrates available for studies of this type has prevented a systematic experimental study of substrate-induced effects. 
Metallic substrates

The large corrugation in the adsorbate-substrate binding energy of insulating substrates arises because the electrons near the surface are highly localized. Metallic substrates are known to provide approximately one order of magnitude reduction in the corrugation because of the de-localized nature of many of the electrons in metals. 33 Unfortunately, these de-localized electrons also contribute strongly to the low-temperature heat capacity of metals, which must be subtracted in the experimental measurement of the heat capacity of the adsorbate. Porous metallic substrates can be fabricated with large surface-to-volume ratios, but the resulting surfaces are a complicated assorment of crystal orientations, and are difficult to clean and characterize. Another method, which has been applied in this case, is to use well-oriented metal films deposited on materials with very small low-temperature heat capacity.

This group has been heavily involved in the development of infrared cornposite bolometers for experimental investigations of the mm-wave cosmic background. Jetectors of this type are the most sensitive broadband detectors of far-infrared radiation. The requirements for the construction of sensitive detectors of this type are extremely low-heat capacity substrates, responsive, low-noise resistance thermometers with low heat capacity, and well-defined but weak thermal contact with a temperature reservoir. To convert a composite bolometer with these characteristics into a sensitive calorimeter, a method for applying a known quantity of heat must be added. For simplicity, we have chosen to use evaporated metallic films as resistive heaters for this purpose. The great sensitivity of these structures to absorbed infrared radiation is then translated into great sensitivity to changes in heat capacity.

The calorimeters are coated on one surface with a $\sim 1000 \AA$ evaporated film, which serves as the substrate for physisorption of ${ }^{4} \mathrm{He}$. One important feature of this approach is 
that it allows investigations on a wide variety of adsorption substrates in the same apparatus. In particular, it allows metallic substrates to be used for the first time.

\section{Film uniformity}

As described above, the uniformity of the adsorption substrate is very important. Since the metallic film used on these calorimeters cannot be single-crystal, and is not cleaned after deposition, there is some concern as to its uniformity. Rough $\mathrm{Ag}$ films evaporated on low-temperature surfaces have been widely investigated for their contribution to large enhancements in the Raman signals obtained from adsorbed CO molecules. Recent scanning tunneling microscopy (STM) investigations of Ag films condensed on room-temperature sapphire substrates have shown them to be composed of (111) crystallites, approximately $500 \AA$ in extent, separated by compact boundary regions as shown in Fig. 2.34 These crystallites are comparable in size to the crystallites in exfoliated graphite. Sophisticated in-situ characterization of the surfaces used in these experiments is not possible because of requirements for shielding from room-temperature radiation.

A series of experiments on ${ }^{4} \mathrm{He}$ submonolayers adsorbed on these $\mathrm{Ag}$ films have been completed and is described below. Those measurements show no evidence for corrugation or defect-induced effects. The absence of such effects in those measurements should be taken as strong evidence that these films are highly uniform to ${ }^{4} \mathrm{He}$ physisorption.

\section{Goal of the study}

There have been many measurements of the thermodynamics of monolayers adsorbed on graphite and related substrates. We have measured the properties of films 


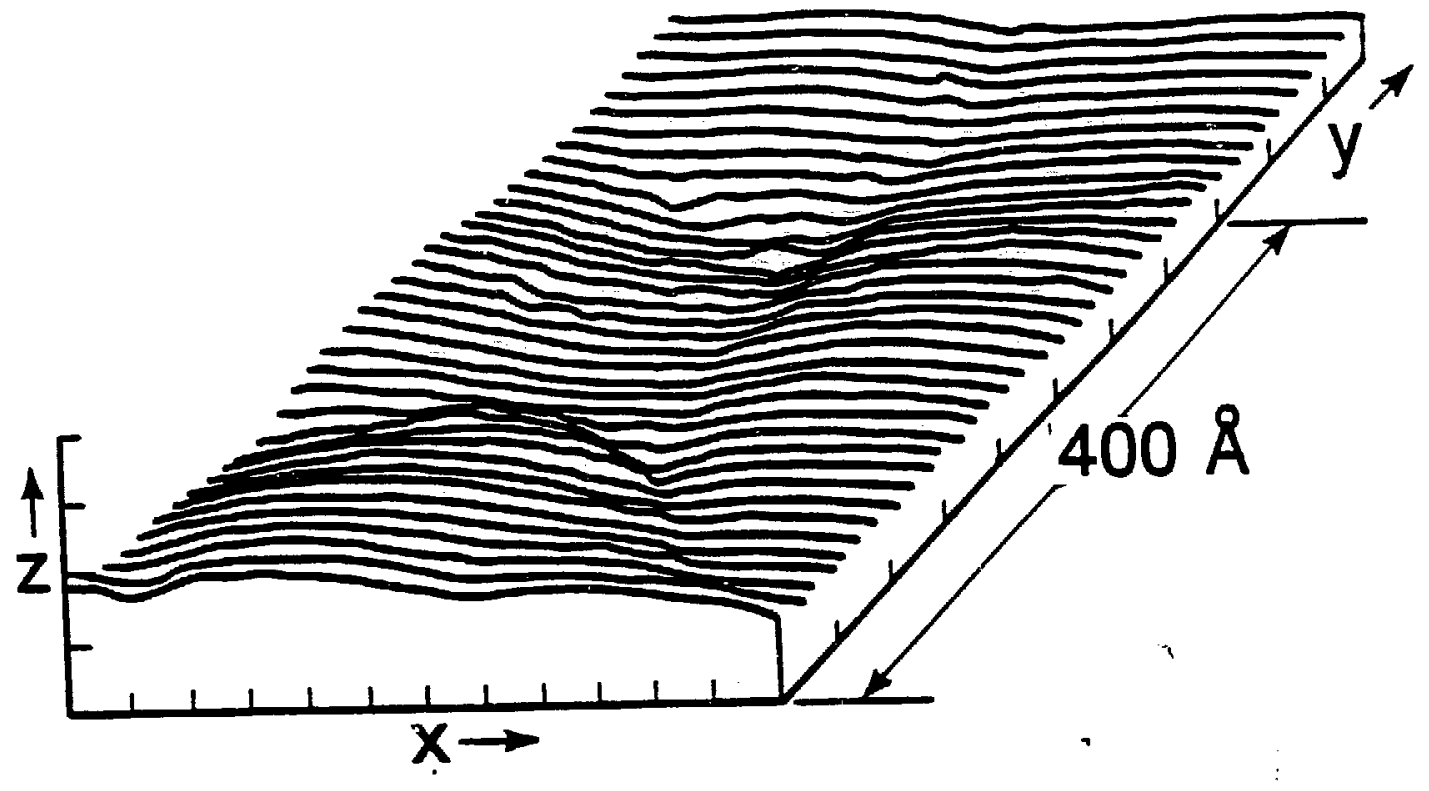

XBL $893-5063$

Figure 2 Scanning tunneling microscope profile of $\mathrm{Ag}$ film deposited on sapphire at room temperature, from Gimzewski, et al (1985). 
adsorbed on metallic substrates in an attempt to understand the role of the substrate in determining the behavior of the adsorbate. In the range of temperatures accessible to our apparatus, ${ }^{4} \mathrm{He}$ and para- $\mathrm{H}_{2}$ monolayers exhibit interesting characteristics. Tha nature of the solid phases and their melting transitions are of obvious interest. The behavior of these monolayers in the presence of the metallic substrate is expected to differ from that observed on insulating substrates. In particular, we expect the metallic substrate will provide a less corrugated physisorption environment. The reduction of the corrugation will certainly affect the commensurate phases. Other changes in the character of the substrate may affect the formation of the commensurate solids. Since experiments on metallic substrates have not been performed before, there will likely be a number of unexpected results. It is this possibility for the exploration of new phenomena that was the principle motivation for these experiments. 
References

1 K.J. Strandburg, Rev. Mod. Phys. 60, 161 (1988).

2 J.G. Dash, Films on Solid Surfaces (Academic Press, New York, 1975).

3 D.L. Goc.jstein, J.G. Dash, and W.D. McCormick, Phys. Rev. Lett. 15, 447 (1965).

${ }^{4}$ E. Giamello et al, Surf. Sci. 49, 401 (1975).

5 M. Bretz, et al, Phys. Rev. A 8, 1589 (1973).

6 J.M. Kosterlitz and D.J. Thouless, J. Phys. C 6, 1181 (1978).

7 B.I. Halperin and D.R. Nelson, Phys. Rev. Lett. 41, 121 (1978).

8 A.P. Young, Phys. Rev. B 19, 1855 (1979).

9 S.T. Chui, Phys. Rev. B 28, 178 (1983).

10 R.E. Ecke, J.G. Dash, R.D. Puff, Phys. Rev. B 26, 1288 (1982).

11 K.J. Strandburg et al, Phys. Rev. Lett. 55, 2226 (1985).

12 M. Bretz, Phys. Rev. Lett. 38, 501 (1977).

13 R.L. Siddon and M. Schick, Phys. Rev. A 9, 907 (1974).

14 G. Derry et al, Surf. Sci. 87, 629 (1979) and G. Boato et al, Surf. Sci. 80, 518 (1979).

15 W.E. Carlos and M.W. Cole, Phys. Rev. B 21, 3713 (1980).

16 G.B. Huff and J.G. Dash, J. Low Temp. Phys. 24, 155 (1976).

17 J.P. McTague et al, Phys. Rev. B 25, 7765 (1982).

18 A.D. Migone, Z.R. Li, M.H.W. Chan, Phys. Rev. Lett. 53, 810 (1984).

19 M.J. Nielson et al, Phys. Rev. B 35, 1419 (1987).

20 E.D. Specht et al, Phys. Rev. B 30, 1589 (1984).

21 P.A. Heiney et al, Phys. Rev. B 28, 6416 (1983).

22 N.J. Colella and R.M. Suter, Phys. Rev. B 34, 2052 (1986). 
23 N. Greiser et al., Phys. Rev. Lett. 59, 1625 (1987).

24 A.J. Jin, M.R. Bjurstrom, M.H.W. Chan, Phys. Rev. Lett. 62, 1372 (1989).

25 I.F. Silvera, Rev. Mod. Phys. 52, 393 (1980).

26 R.E. Palmer and R.F. Willis, Surf. Sci. 179, L1 (1987).

27 Ph. Avouris, D. Schmeisser, and J.E. Demuth, Phys. Rev. Lett. 48, 199 (1982). and

D. K. Veirs, Private Communication.

28 M. Bretz and T.T. Chung, J. Low Temp. Phys. 17, 479 (1974).

29 F.C. Motteler and J.G. Dash, Phys. Rev. B 31, 346 (1985).

$30 \mathrm{~J}$. Cui and S.C. Fain, Phys. Rev. B in press (1989).

31 S.B. Crary and O.E. Vilches, Phys. Rev. Lett. 38, 973 (1977).

32 C. Schwartz and M.W. Cole, Phys. Rev. B 34, 1250 (1986).

33 J. Ungaris et al. Surf. Sci. 109, 522 (1981).

34 J.K. Gimzewski et al, Phys. Rev. Lett. 55, 951 (1985) 


\section{Signal/noise ratio for ideal ac calorimeters}

\section{Choice of Measurement Technique}

The technique chosen for heat capacity measurements depends on the intrinsic time constants of the calorimeter. For calorimeters constructed with exfoliated graphite or other porous adsorption media, the internal thermal time constants can be $10^{2}$ to $10^{3}$ seconds. For this reason, the heat capacity of many adsorbed monolayer systems has been measured by slow adiabatic techniques.

In our experiment, the heat capacity of the calorimeter is made very small so that the contribution of the adsorbate can be detected. At the same time, the thermal conductance to the heat sink must be large enough to allow measurements near the minimum operating temperature of the cryostat. The resulting thermal time constant of our calorimeter is between $10^{0} \mathrm{~s}$ and $10^{-3} \mathrm{~s}$, so is appropriate for ac calorimetry. 1

\section{Calorimeter Optimization}

In order to optimize the measurement, we consider an ideal calorimeter with heat capacity $C$ isolated from a heat sink at temperature $T_{0}$ by a thermal conductance $G$. The calorimeter has a calibrated thermometer with resistance $R(T)$ and a heater with resistance $\mathrm{R}_{\mathrm{H}}$. It is assumed that the intermal time constants of all elements of the calorimeter including the thermometers are much shorter than C/G.

Under normal operating conditions, the thermometer is biased with a dc current $\mathrm{I}=$ $V_{D C} / R_{T}$, and the heaters are biased with an ac voltage $V_{H}$ at frequency $\omega / 2$. The temperature of the calorimeter oscillates at the frequency $\omega$ with amplitude 


$$
T_{A C}=\frac{V_{H^{2}}}{2 R_{H}\left[(\omega C)^{2}+(G)^{2}\right]^{1 / 2}}
$$

Due to the temperaure dependence of the resistance, the voltage across the thermometer oscillates with amplitude $V_{A C}=V_{D C} \alpha T_{A C}$ where $\alpha$ is defined as $\partial \log \left(R_{T}\right) / \partial T$. When $\omega C / G \gg>1$, the heat capacity can be deduced from a measurement of $V_{A C}$.

We can calculate the errors induced in the measured signal from fundamental noise sources. Johnson noise in the thermometer has a spectral density ${ }^{2}$

$$
\left(V_{\mathrm{J}}\right)^{2}=4 \mathrm{k}_{\mathrm{B}} \mathrm{R}_{\mathrm{T}} \mathrm{T} \text {, }
$$

where $k_{B}$ is Boltzmann's constant.

Energy fluctuations also contribute to the noise in a calorimeter. These arise from the statistical fluctuations in the rate of arrival of phonons from the heat sink and are also known as phonon noise. ${ }^{3}$ Because of the temperature dependence of the resistance of the thermometer, these fluctuations contribute to the voltage noise. The resulting voltage fluctuations have a spectral density

$$
\left(V_{P}\right)^{2}=\frac{4 k_{B} T^{2} G\left(V_{D C} \alpha\right)^{2}}{(\omega C)^{2}+(G)^{2}}
$$

The voltage fluctuations (2) and (3) are statistically independent and their spectral densities are additive. The ratio of noise voltage to signal voltage is

$$
\frac{V_{N^{2}}}{V_{A C^{2}}}=\frac{16 k_{B} T \Delta F R_{H^{2}}\left[(\omega C)^{2}+(G)^{2}\right]}{V_{D C^{2} \alpha^{2} V_{H^{4}}}}\left[R_{T}+\frac{G T V_{D C} \alpha^{2}}{(\omega C)^{2}+(G)^{2}}\right]
$$


To optimize the sensitivity of an ac calorimeter, the ratio of noise to signal is minimized subject to some two experimental constraints:

1) The maximum permissible amplitude of the oscillation, $T_{A C}$, which sets the temperature resolution, is determined by the experiment to be done. This constraint is introduced by replacing $\left((\omega C)^{2}+(G)^{2}\right)$ in Eq. 6 with $\left(V_{H^{2}} / 2 T_{A C} R_{H}\right)^{2}$. This leaves the ratio (4) in the form

$$
\frac{V_{N}^{2}}{V_{A C}^{2}}=4 k_{B} T\left(\frac{R_{T}}{V_{D C^{2} \alpha^{2} T_{A C}}}+\frac{4 G T_{H_{H}}^{2}}{V_{H^{4}}^{4}}\right)
$$

2) The temperature of the calorimeter is also specified by the measurement to be done. Therefore, the temperature difference between the calorimeter and the heat sink determines the maximum total measurement bias power in terms of the thermal conductance to the heat sink.

$$
\left(T-T_{0}\right)=\frac{V_{H}^{2}}{2 R_{H} G}+\frac{V_{D C}}{R_{T} G}=\Delta T_{H}+\Delta T_{B}
$$

Here, $\Delta T_{H}=V_{H} 2 / 2 R_{H} G$ is the time average temperature rise due to the ac bias on the heater, and $\Delta T_{B}=V_{D C} / R_{T} G$ is the temperature rise due to the dc bias on the thermometer. With these substitutions, Eq. 4 takes the form

$$
\frac{V_{N}^{2}}{V_{A C}^{2}}=\frac{4 k_{B} T}{G}\left(\frac{1}{\alpha^{2} T_{A C}^{2} \Delta T_{B}}+\frac{T}{\Delta T_{H^{2}}}\right)
$$

Of the terms in the parentheses, only $\Delta \mathrm{T}_{\mathrm{B}}, \Delta \mathrm{T}_{\mathrm{H}}$, and $\alpha$ can be freely adjusted. The other terms in the parentheses are determined prior to the measurement. In principle, one can 
improve the sensitivity at a particular temperature by choosing thermometers with very large $\alpha$. However, rapid variations in the resistance of the thermometer with temperature can make measurements over a broad temperature range exceedingly difficult. In practice, values of $\alpha$ of order $1 \mathrm{~K}^{-1}$ provide a compromise between sensitivity and versatility.

Given values of $T, T_{A C}$, and $\alpha$, the expression in the parentheses can easily be minimized subject to the constraint of Eq. 6. The appearance of $G$ in the denominator of Eq. 7 indicates that the noise in an ideal calorimeter can be reduced arbitrarily by increasing G. For non-ideal calorimeters, however, the limits imposed by internal time constants within the calorimeter must be considered. As Eq. 1 shows, when $\mathrm{G}$ is increased, $\mathrm{V}_{\mathrm{AC}}$ becomes independent of $\mathrm{C}$ uniess $\omega$ is also increased. Internal time constants in real calorimeters set a limit to the highest frequencies at which Eq. 1 can be used. Internal time constants can arise from electrical RC effects, or thermal relaxation within the elements of the calorimeter. A much more detailed thermal analysis would be required to interpret data at frequencies comparable to the inverse of the intermal time constants.

Further limits to the sensitivity of an ac calorimeter are imposed by the accuracy of the devices which record the electronic signals from the experiment. At present, 16-bit A-D converters are commercially available for most computers, and 18-bit devices are starting to become available.

Finally, the presence of slow thermal drifts can reduce the accuracy of measurements that depend on the difference between heat capacities measured at different times. Because of the strong temperature dependence of the heat capacity of the calorimeter, variations in the temperature of the calorimeter will appear as large variations in signal. As a result, active thermal regulation of the calorimeter is necessary. The accuracy 
and stability of the regulation scheme must be optimized to minimize its contribution to measurement noise.

In summary, we have seen that the sensitivity of an ideal calorimeter can be arbitrarily increased. Practical limits to the sensitivity are imposed, however, by the internal time constants of the calorimeter, the accuracy of the ADC, and the stability of the thermal regulation. For the calorimeters described in this report, it is usually the case that for operation at frequencies just below the internal relaxation frequencies, the sensitivity is limited by the accuracy of the thermal regulation. Nevertheless, enough sensitivity has been obtained to detect a small fraction of a monolayer of ${ }^{4} \mathrm{He}$ adsorbed on the metallic surface of the calorimeter. 4 
References

1 P.F. Sullivan and G. Seidel, Phys. Rev. 173, 679 (1968).

2 R.A. Smith, F.E. Jones, R.P. Chasmar, The Detection and Measurement of Infra-red Radiation, (Oxford niversity Press, London, 1968) P. 185

3 Tbid p. 212.

4 T.W. Kenny, P.L. Richards, Bull. Am. Phys. Soc. 33, 264 (1988). 


\section{ExperimentaL_Aonaratus}

\section{Calorimeter}

The design of the calorimeter used in these experiments is based on that of a composite bolometer which is the most sensitive available direct detector of far-infrared radiation. ${ }^{1} \mathrm{~A}$ bolometer operates by measuring the temperature rise induced by the absorption of radiation. In attempting to improve the sensitivity of composite bolometers, workers have developed resistance thermometers with low noise, low heat capacity, and high sensitivity. These advances have been incorporated directly into the design of the microcalorimeters described here. A composite bolometer can be converted to a calorimeter by attaching a resistive heater. The heat capacity can then be calculated from the absorbed power and the temperature rise.

A drawing of the calorimeter is shown in Fig. 3. The calorimeter is constructed on a $125 \mu \mathrm{m}$ sapphire substrate which has been optically polished on one surface. The use of thinner substrates would provide more sensitivity, but would make the calorimeters more fragile. Sapphire has been chosen as the substrate material because of its small heat capacity at ${ }^{4} \mathrm{He}$ temperatures. A pair of commercially available $\mathrm{NiCr}$ films deposited on $\mathrm{Si}$ wafers serve as resistive heaters. ${ }^{2} \mathrm{Six}$ leads of $25 \mu \mathrm{m}$ diameter $\mathrm{Cu}$ wire, two thermometers and two heater packages are attached to the substrate with an Ag-filled conductive epoxy. ${ }^{3}$ The $\mathrm{Cu}$ leads provide the thermal conductance to the heat sinks. Two heaters are required; one is used to apply the AC power, and the other is used to actively regulate the average temperature of the calorimeter. In principle, a single heater could be ac and dc biased simultaneously. This can complicate the measurement, because it would induce an oscillation at half the measurement frequency, whose amplitude depended on the regulation current. Because of nonlinearities in the thermometer resistance, as well as electrical cross- 


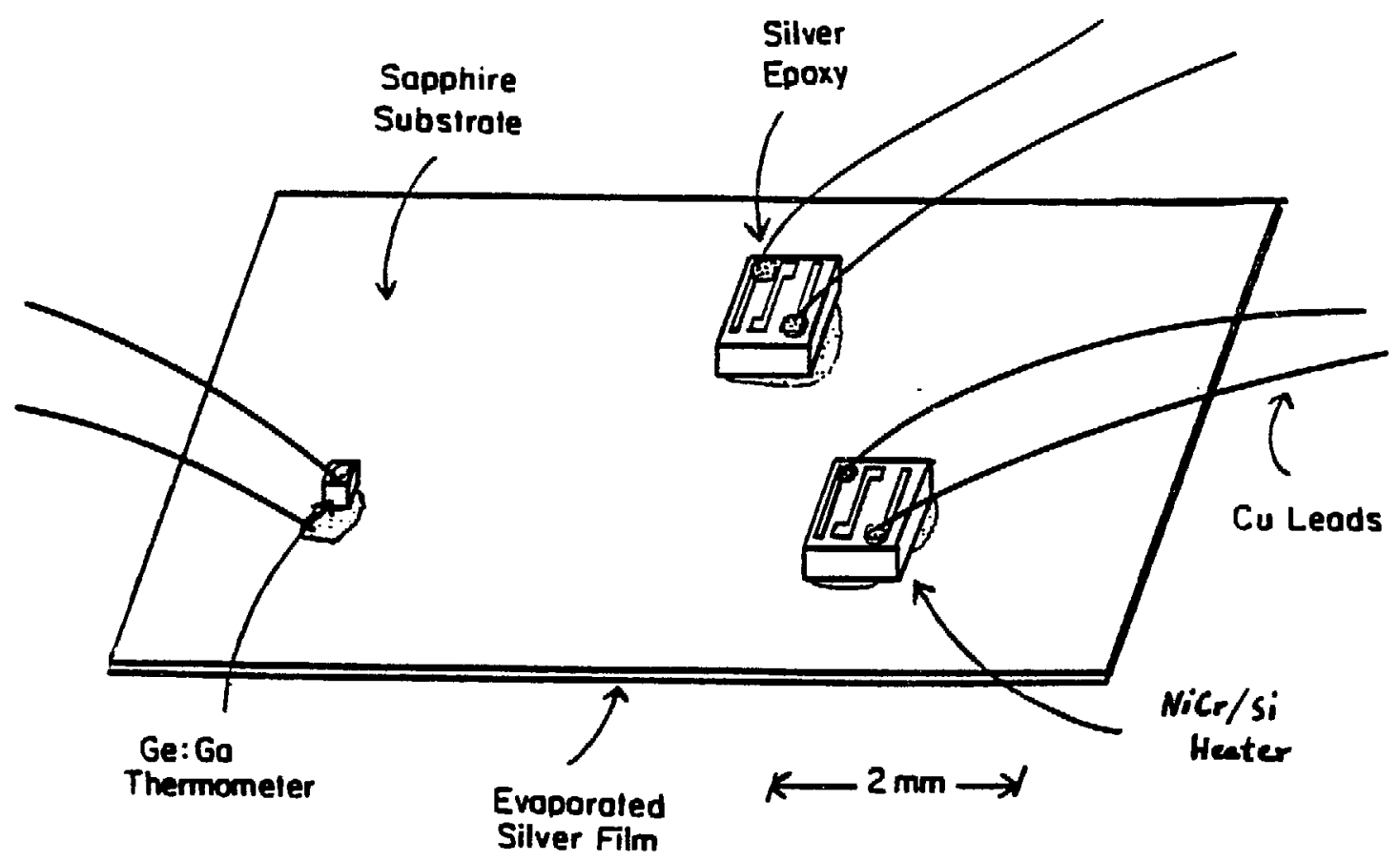

Figure 3 Schematic drawing of the calorimeter used in these studies. The Ge:Ga thermometer, $\mathrm{NiCr}$ heaters and $\mathrm{Cu}$ wires are attached to the back of the sapphire substrate with conductive epoxy. Ag films are deposited in situ on the front of the sapphire. 
talk, some distortion of the signal at the measurement frequency might result. For this reason, two separate heaters are used.

The thermometers are made from single crystal ultrapure Ge which has been doped with Ga acceptors and compensated with As donors to the concentrations shown in Table 1 by neutron transmutation (NTD). The preparation of these thermometers is described elsewhere. ${ }^{4}$ They are useful in this application because of their low heat capacity and large $\alpha$. Electrical contacts which contribute no excess noise are made by $\mathrm{B}^{+}$ion implantation and $\mathrm{Au} / \mathrm{Cr}$ metallization. Thermometers as small as $(100 \mu \mathrm{m})^{3}$ have been fabricated with resistances that are comparible with the current and voltage noise of commercially available preamplifiers.

The temperature dependence of the resistance of the thermometers is measured for each calorimeter before installation in the UHV system. The calorimeters are submerge. $\mathrm{I}$ directly in liquid-4 $\mathrm{He}$, which is slowly cooled from $4.2 \mathrm{~K}$ to $1.4 \mathrm{~K}$ by pumping the bath. The vapor pressure over the liquid- ${ }^{4} \mathrm{He}$ is monitored by a capacitance manometer. The resistance of the thermometer and the vapor pressure of the liquid- $-4 \mathrm{He}$ are recorded manually at several points throughout this temperature range. The temperature dependence of the resistivity for all of the thermometer types used in these measurements is shown in Fig. 4. We have verified that the resistance of the thermometers does not change as a result of thermal cycling by repeating several of the calibrations.

Despite their many advantages, some properties of these thermometers cause difficulties with the measurement ${ }^{5}$ The dominant conduction mechanism in these materials at ${ }^{4} \mathrm{He}$ temperatures is variable-range hopping. This mechanism has been carefully studied, 
Table 1

\begin{tabular}{c|l|l|l|l} 
& & & \\
Dopant & NTD-1 & NTD-2 & NTD-3 & NTD-4 \\
\hline $\mathrm{Ga}$ & $2.21 \times 10^{15}$ & $4.41 \times 10^{15}$ & $6.62 \times 10^{15}$ & $9.94 \times 10^{15}$ \\
$\mathrm{As}$ & $6.28 \times 10^{14}$ & $1.26 \times 10^{15}$ & $1.89 \times 10^{15}$ & $2.83 \times 10^{15}$ \\
$\mathrm{Se}$ & $9.2 \times 10^{13}$ & $1.6 \times 10^{14}$ & $2.5 \times 10^{14}$ & $3.8 \times 10^{14}$ \\
$\mathrm{~N}_{\mathrm{a}-\mathrm{N}_{\mathrm{d}}}$ & $1.49 \times 10^{15}$ & $2.99 \times 10^{15}$ & $4.48 \times 10^{15}$ & $6.73 \times 10^{15}$
\end{tabular}

Table 1 Dopant concentrations in the NTD samples used in this study. All other impurity concentrations are below 1013. All concentrations in $\mathrm{cm}^{-3}$.

both experimentally and theoretically. The resistance is a function of both the temperature and the applied electric field. Because of the field dependence, resistivity measurements must be made with either very small electric fields, or exactly the same fields as used for calibration. Time constants in excess of $10^{-2} \mathrm{~s}$ due to weak electron-phonon coupling have also been observed in variable range hopping systems.6,7 Experiments on NTD Ge doped to $\mathrm{N}_{\mathrm{A}}=10^{16} \mathrm{~cm}^{-3}$, however, have shown no evidence for internal time constants at frequencies as high as $10^{3} \mathrm{~Hz}$. In addition, the electric field effects are less significant in more heavily doped thermometers, as less voltage is required to achieve a given bias power. All of these results are discussed at length elsewhere. ${ }^{5}$ We have assembled many calorimeters with heavily doped NTD thermometers and, with care, obtained reliable results. 


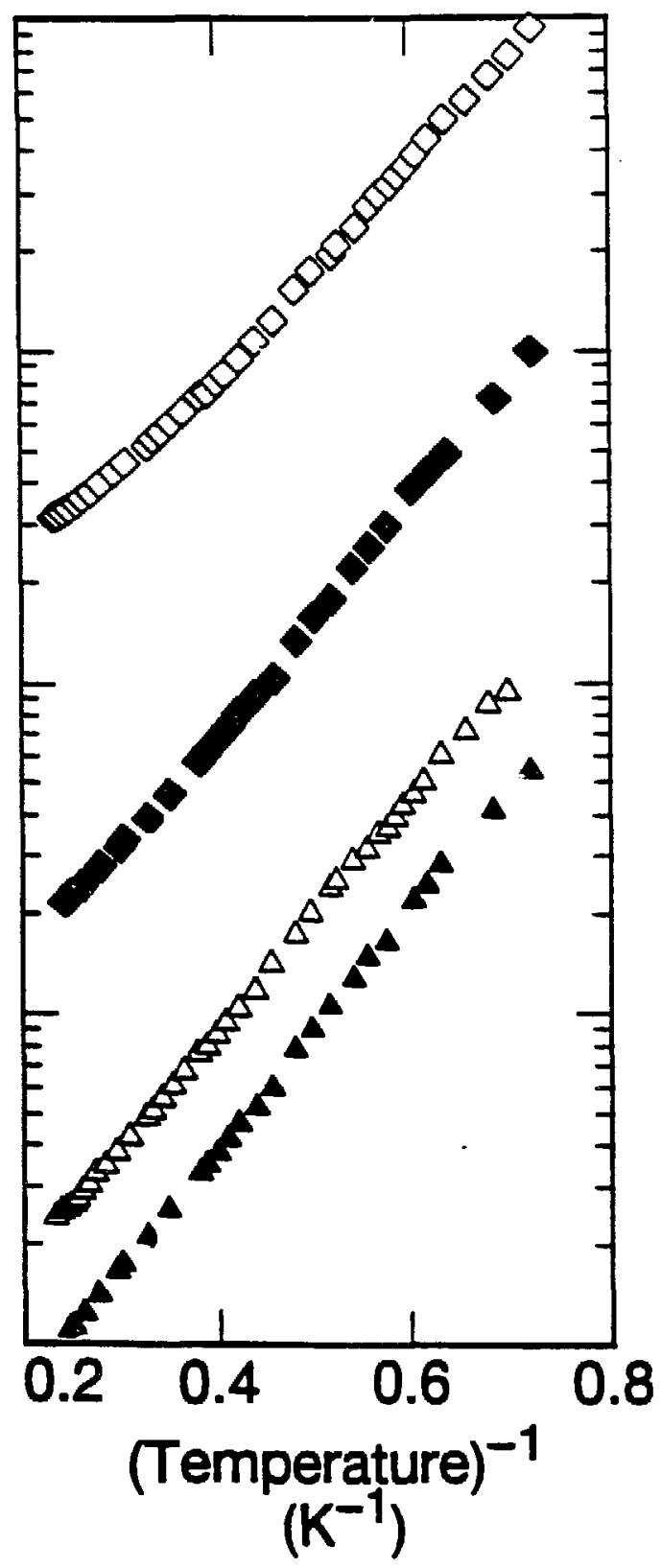

XBL 885.7424A

Figure 4 Plots of the log of the resistivity of all four thermometers measured as a function of (Temperature) $)^{-1}$. 
The thermal time constant of each calorimeter must be measured to verify that, at the measurement frequency, the signal is directly related to the heat capacity. A fixed ac heater bias and de thermometer bias are applied to the calorimeter. The amplitude and phase of the voltage oscillation of the thermometer are then measured as a function of the frequency of the oscillation and compared with the relation

$$
T_{A C}=\frac{P_{A C}}{G+i \omega C}
$$

where $G$ is the thermal conductance to the heat sink, and $P_{A C}$ is the amplinude of the oscillating bias power. The product of the amplitude of the voltage oscillation and the frequency of oscillation is plotted as a function of oscillation frequency in Fig. 5. In such a plot, the acceptable operating frequencies are on the plateau of the curve. For the figure, the acceptable frequencies are between $300 \mathrm{~Hz}$ and $1 \mathrm{kHz}$. At higher frequencies, additional time constants further steepen the rolloff. For the measurements reported here, the capacitance of the heat sinks and the resistance of the thermometers are responsible for the additional rolloff. The low heat capacity and high thermal conductivity of the various elements used in the construction of the calorimeter insure that the internal thermal time constants of the calorimeter are very short. An indirect measurement of the time constants of calorimeters of this type was carried out as part of an earlier experiment. Infrared composite bolometers that were constructed in a manner similar to these calorimeters were used to detect far-infrared (FIR) picosecond pulses. The pulse deposits energy into a thin Bi film on one side of the substrate, and is detected through the change in resistance of the thermometer, which is mounted on the other side of the substrate. A typical oscilloscope trace of the response of the detector to such a pulse is shown in Fig 6. Since the FIR pulse is very short on the ime scale of the figure, the rise time is due to the time constants of the 


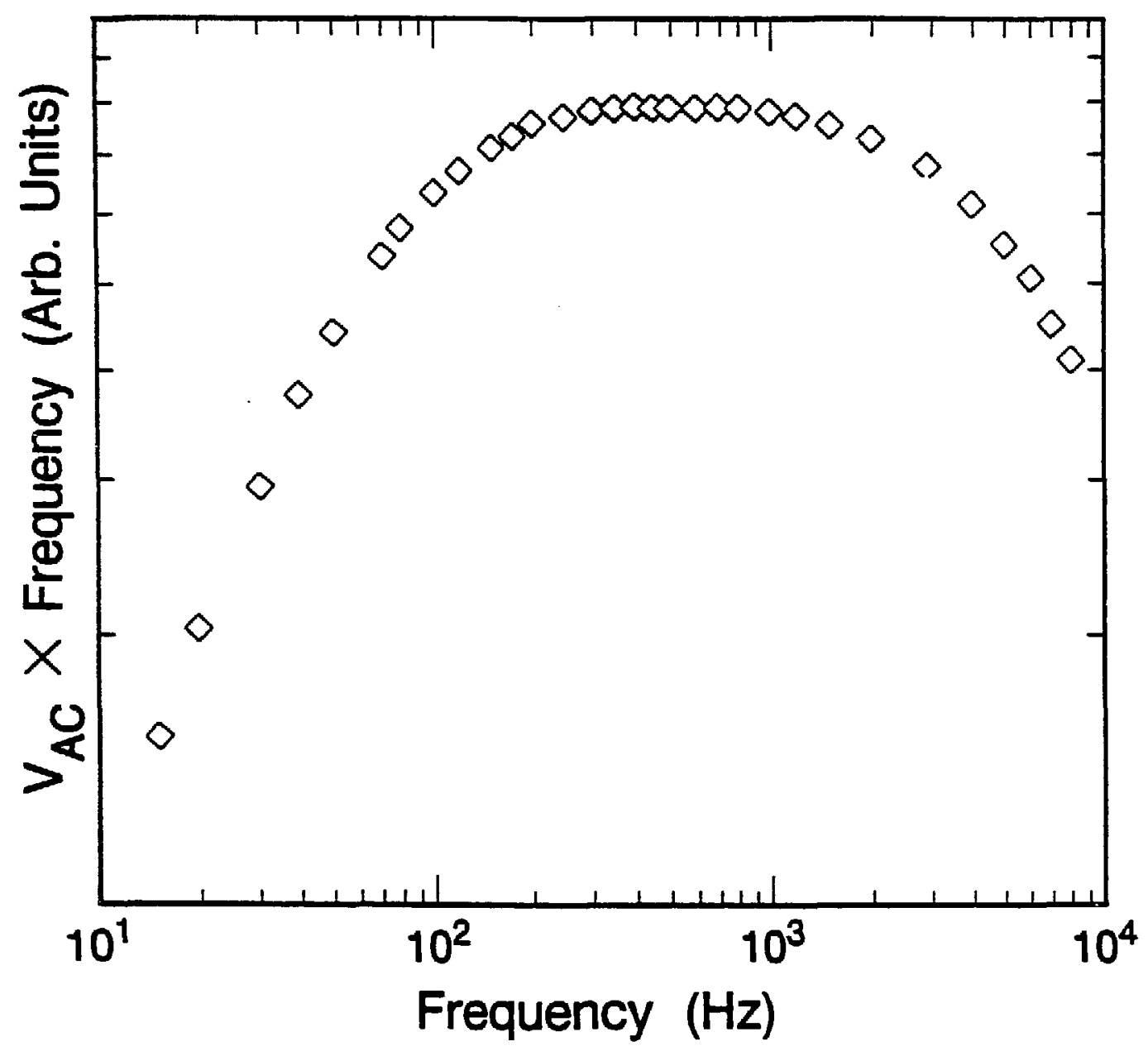

XBL 893-5062

Figure 5 Measurement of the product of the amplitude of the temperature oscillation and the frequency of the oscillation as a function of the frequency of the oscillation. The plateau indicates the region in which the amplitude of the temperature oscillation is directly related to the heat capacity. 

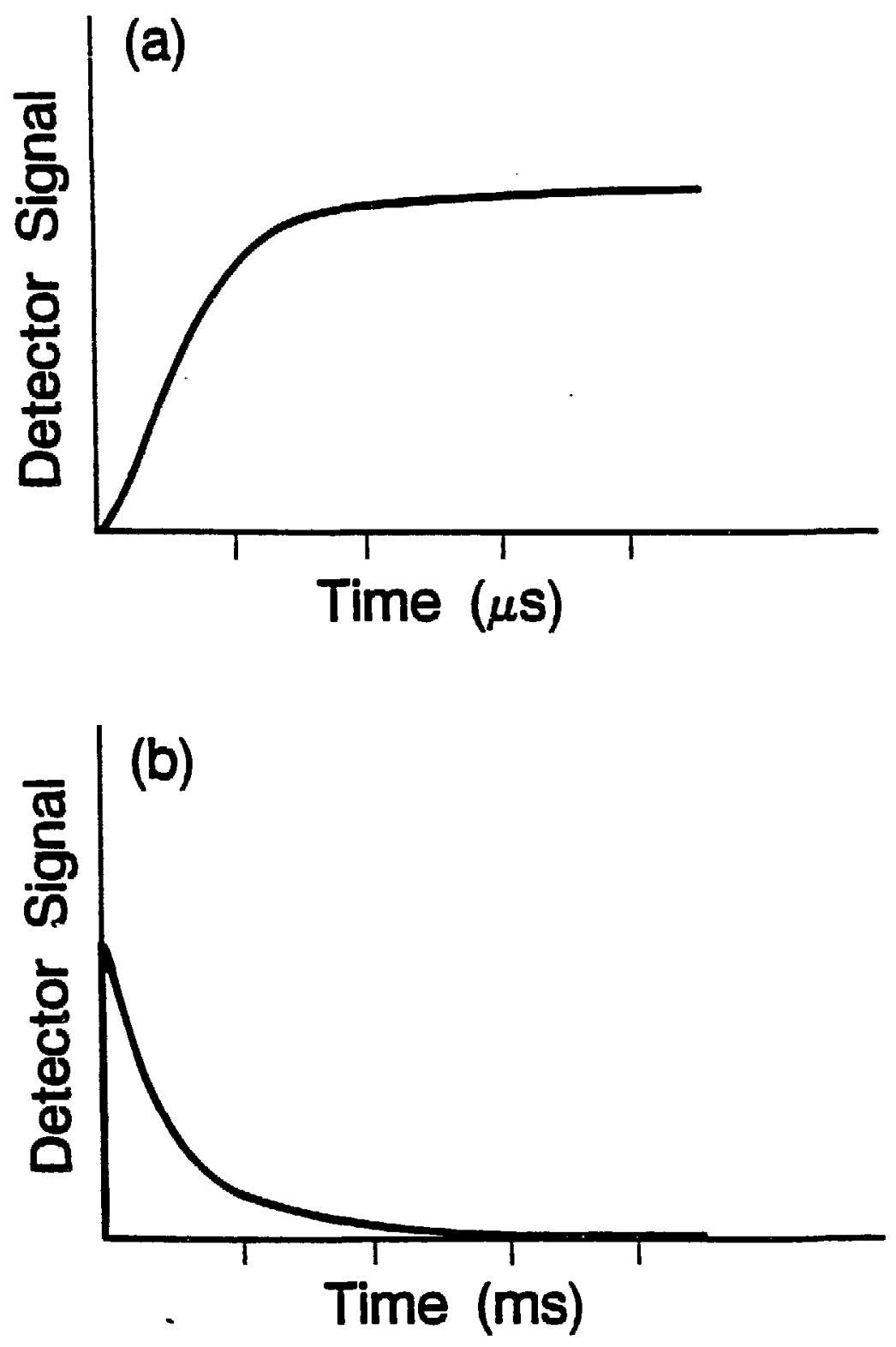

XBL 891-5020

Figure 6 Voltage response of a de-biased composite infrared bolometer to a FIR picosecond pulse. The time for the bolometer to reach internal thermal equilibrium is several $\mu s$, as shown in (a), while the time for the heat to escape through the thermal contact to the reservoir is ms as showr in (b). 
detector and the assorted electronics. From the figure, we can see that the internal time constants of such detectors are no longer than several $\mu \mathrm{s}$. Therefore, ac calorimetry on the ms time scale is reasonable with these structures.

The calorimeter is mounted within an aluminum and stainless steel frame which constrains its motion with negligible thermal contact. The frame also masks the evaporation so that metallic paths from the front surface of the calorimeter to other parts of the apparatus do not exist. This is important because the calorimeter is always warmer than its surroundings, and metallic paths might allow migration of the adsorbed atoms off of the calorimeter during the measurement. The frame is attached to Cu posts on the mount with stainless steel screws. A drawing of the frame and the mount is shown in Fig. 7. After assembly, the calorimeter, frame and mount are submerged in ${ }^{4} \mathrm{He}$ for thermometer calibration. The mount is then attached to the end of the cold finger of the cryostat with stainless steel screws as is shown in Fig. 8. A $12 \mu \mathrm{m}$ Au foil is placed between the mount and the cold finger to improve thermal contact.

An earlier mount used layers of teflon tape to ensure thermal isolation berween the frame and the calorimeter. During heating of the calorimeter to room temperature for deposition of fresh, oriented $\mathrm{Ag}$ films, the thermal contact through the teflon tape became quite large, requiring power in excess of 1 watt to be dissipated in the heater films. After a small number of such treatments, the Bi film heaters used in these early calorimeters usually became unstable, requiring the construction of a new calorimeter. Removal of the teflon tape reduced the thermal contact at high temperatures by $20 x$. 


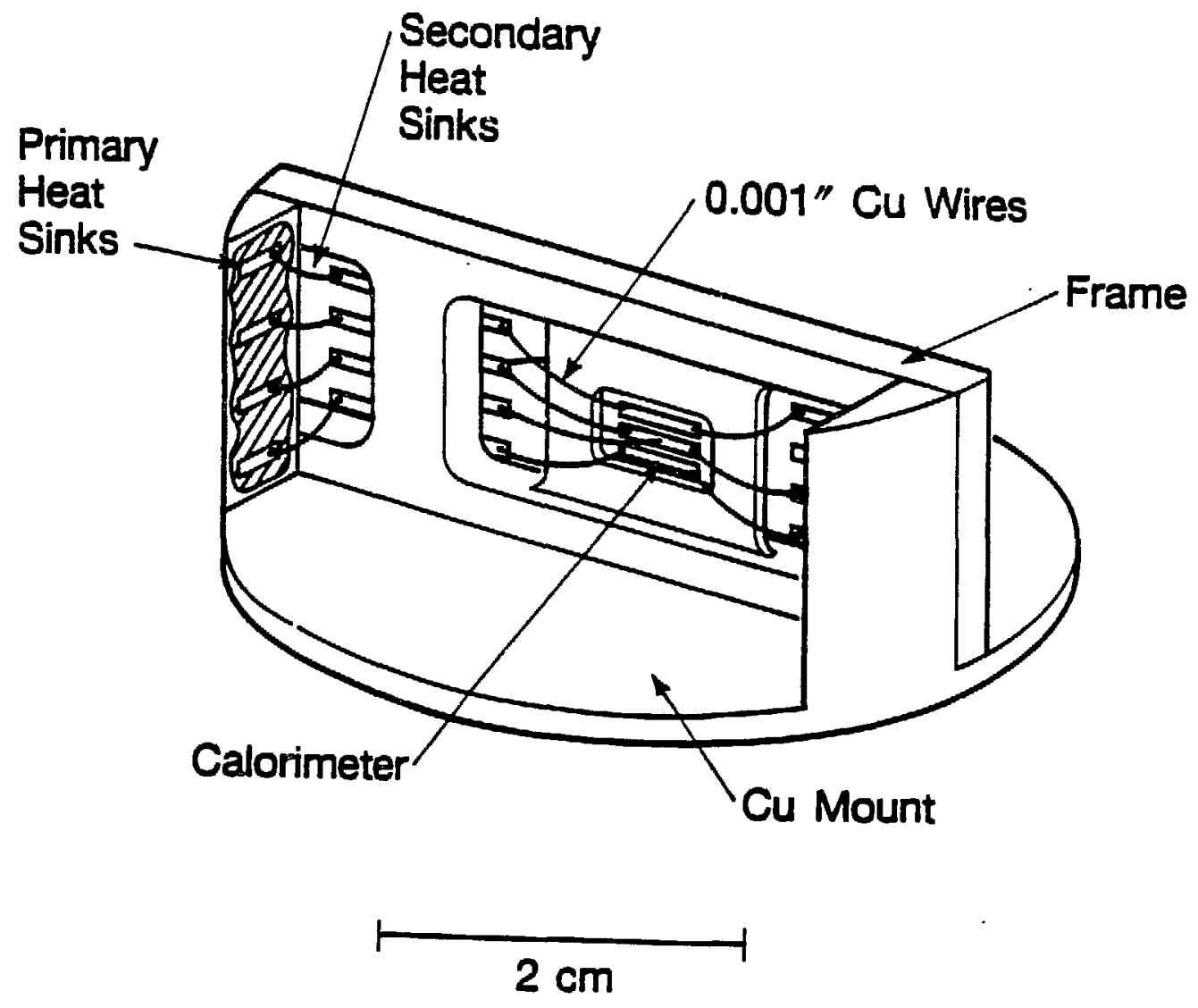

XBL 8810-7636

Figure 7 The calorimeter mount and frame with the calorimeter in place. The calorimeter is constrained within the frame, which also serves as a mask for in-situ evaporation of clean surfaces. The mount is attached to the cold finger in the UHV system and can be cooled to $1.6 \mathrm{~K}$ during measurements. 


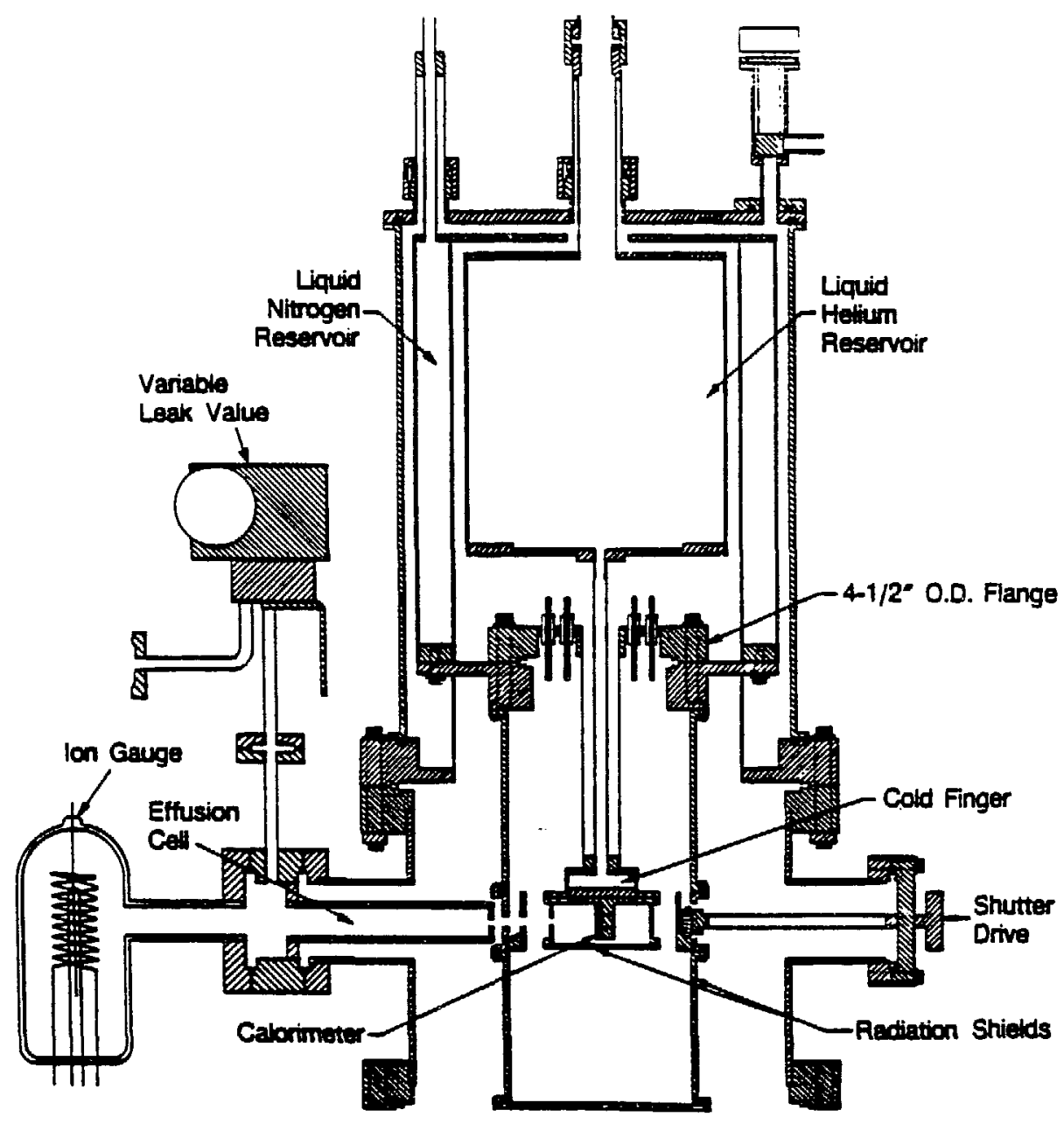

Figure 8 A cross-section of the cryostat and UHV system. The upper part includes storage canisters for the liquid nitrogen and pumped liquid ${ }^{4} \mathrm{He}$. The lower half is an ionpumped vacuum chamber, allowing surface preparation and heat capacity measurements at pressures as low as 10-12 torr. The evaporation source, effusion cell, and mass spectrometer are mounted on flanges at the level of the sample. Evaporation of fresh films, and dosing of the calorimeter with adsorbates takes place through a series of holes in the radiation shields. One of the liquid nitrogen temperature shields can be rotated to prevent room temperature radiation from reaching the sample directly during measurements. 
A radiation shield mounted on the cold finger allows the calorimeter to be operated at temperatures as low as $1.6 \mathrm{~K}$. The inside of this shield has been coated with colloidal graphite to absorb room-temperature radiation which leaks around the shield and to provide additional pumping of background ${ }^{4} \mathrm{He}$ gas. Further reductions in temperature are only possible with the aid of better refrigeration, such as with ${ }^{3} \mathrm{He}$.

Low thermal conductance stainless steel coaxial cables are used throughout the apparatus. Electrical leads reach the calorimeter through a liquid nitrogen temperature feedthrough into the UHV system. Two stages of heat sinks on the mount provide thermal isolation for the calorimeter. The heat sinks are made from $1.5 \times 10.0 \times .08 \mathrm{~mm}^{3} \mathrm{Cu}$ plates which are attached with Stycast ${ }^{8}$ epoxy. This epoxy contains small glass spheres which insure electrical insulation between flat, metallic surfaces while providing good thermal contact 9

At ${ }^{4} \mathrm{He}$ temperatures, the heat capacity of the calorimeter is dominated by the sapphire substrate and is proportional to $\mathrm{T}^{3}$. Deviations from this dependence arise below $2.5 \mathrm{~K}$ due to the metallic parts of the calorimeter whose heat capacities have a component proportional to $\mathrm{T}$. The heat capacity of the bare calorimeter is measured as a function of temperature at the beginning of each experiment. After the adsorption of the sample gas, the heat capacity is measured again. Subtraction of these two results yields the heat capacity of the adsorbed sample.

\section{UHV system and cryostat}

A cross section of the UHV system and cryostat used in this experiment is shown in Fig. 8. The upper half of the apparatus is a stainless steel cryostat which contains chambers for liquid nitrogen and liquid ${ }^{4} \mathrm{He}$. The cryostat is evacuated by a $40 \mathrm{l} / \mathrm{s}$ 
turbomolecular pump, and is also cryopumped by the surfaces of the storage chambers. The ${ }^{4} \mathrm{He}$ canister has been wrapped with several layers of aluminum foil, which reduced the radiative heat flow to the bath, thereby extending the hold-time of the apparatus.

The lower half of the apparatus is a commercial ion-pumped UHV system which can be evacuated to $<10^{-9}$ torr without bakeout when ai room temperature. The outer radiation shields are cooled to about $100 \mathrm{~K}$ by conduction from the liquid nitrogen storage chamber in the cryostat. The temperature of these shields can be monitored by measuring the resistance of the several Allen-Bradley $15 \mathrm{~K} \Omega$ resistors that are attached to it at various locations. The cold finger is cooled to $1.6 \mathrm{~K}$ by direct contact with superfluid ${ }^{4} \mathrm{He}$ which flows down a thin tube from the cryostat. During measurement, the pressure near the sample is of order $10^{-12}$ torr due to cryopumping on the radiation shields. A shutter in the nitrogen temperature radiation shields can be rotated to allow the evaporation of a metal film onto the calorimeter substrate. The film thicknesses are monitored by a commercial quartz oscillator near the evaporation filament and are typically of order $1000 \AA$. During dosing, gas enters a room temperature effusion cell from a stainless steel gas line through a leak valve. The pressure within the effusion cell is monitored by an ion gauge. The dosing flux can be calculated from the pressure, the dimensions of the cell, and the distance to the calorimeter. Since the sticking coefficient is not generally known, adsorbate coverages are uncertain, except when they can be deduced from the heat capacity measurement.

Since bakeouts are unnecessary, low melting point materials such as indium can be used between the joints in the radiation shields and in the cold finger. Also, the background pressure in the apparatus is only important during the measurement, when much of it is very cold. This allows the use of many materials on or near the cold finger which are not usually suitable for UHV systems. For example, epoxies ${ }^{32}$ have been used in the construction of heat sinks. Granules of activated charcoal have been attached to the 
cold finger in several places to improve pumping of background ${ }^{4} \mathrm{He}$ gas. Conductive epoxies 28 have been used in calorimeter construction. Various infrared absorbers have been used to coat the radiation shields which surround the calorimeter. The use of these materials has greatly simplified the design and operation of this experiment.

\section{Measurement electronics}

The electronic system used for this measurement can be described in three sections : the ac heater bias, the thermometer bias and readout, and the temperature regulation. A block diagram of the entire measurement circuit is shown in Fig. 9.

The ac heater bias is generated by the reference channel of a lock-in amplifier. ${ }^{10}$ This source was chosen for its excellent amplitude stability and ease of frequency and amplitude adjustment. The amplinude of the ac bias voltage is measured by the same lockin amplifier, allowing small amplitude drifts to be removed from the data. This voltage is applied to one of the heaters on the calorimeter and produces a temperanure oscillation in the calorimeter.

The dc bias for the thermometer is produced by three $9 \mathrm{~V}$ mercury batteries which are connected in series across a $500 \mathrm{k} \Omega 10$-turn wire-wound potentiometer. The center terminal of the potentiometer is attached to a room temperature metal film load resistor in series with the cold thermometer. When used to bias a resistance thermometer whose temperature is oscillating, this stable current produces a voltage with both $\mathrm{dc}$ and ac components. The dc component is directly related to the average temperaure of the calorimeter, while the ac component is used to calculate the heat capacity. The voltage of the thermometer is processed by both a dc preamplifier, ${ }^{11}$ which is low-pass filtered to 


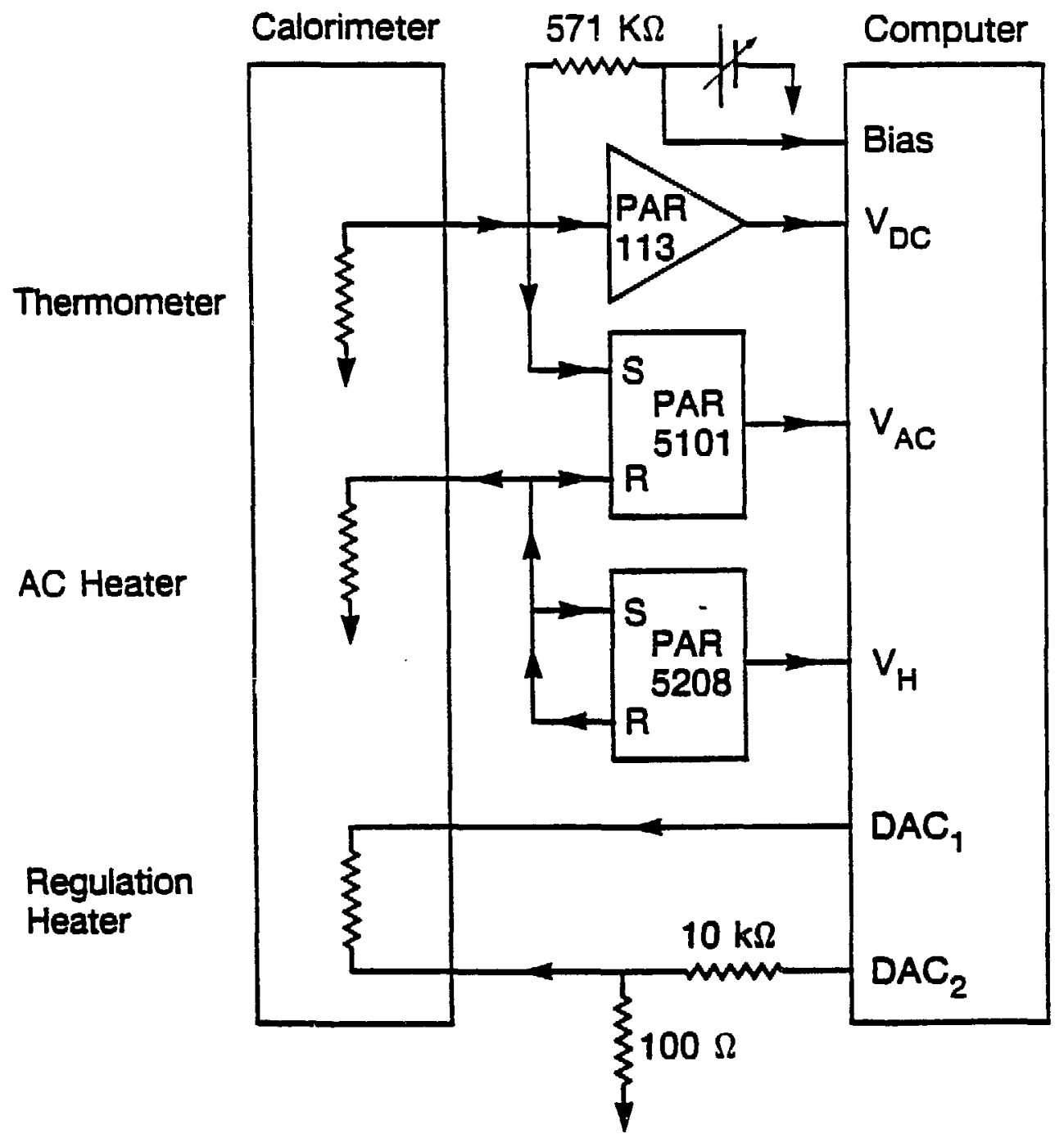

Figure 9 A block diagram of the electronic system. The ac heater bias is obtained from the reference channel of the PAR 5208 lock-in amplifier. The dc thermometer bias is obtained from a series of $9 \mathrm{~V}$ mercury batteries and a wire wound potentiometer. The amplinude of the induced voltage oscillation of the thermometer is measured by the PAR 5101 lock-in amplifier. The de voltage of the thermometer is amplified by a PAR 113 preamplifier and then low-pass filtered to remove the ac components. The outputs are connected to the $A D C$ channels of the computer and recorded. If the temperature of the thermometer drifts during the measurement, the regulation voltage, which is derived from the DAC channels of the computer, is fed back to counteract the drifts. 
remove the voltage oscillation, and by another lock-in amplifier, ${ }^{12}$ which uses the ac bias from the the first lock-in as its reference signal.

An LSI-11 computer with an ADC-DAC board 13 is used to record the measured voltages and produce the thermal regulation voltage which is applied to one of the Bi films on the calorimeter. The resolution of the 12-bit DAC on this board is inadequate for the temperature regulation. An effective resolution of 18-bits is obtained by dividing one of the 12-bit DAC channels by $10^{2}$ and adding it to the other. The voltages are generated by separate DAC channels with a common ground, and so neither of the wires leading to this heater can be externally grounded.

The computer program regulates the temperature and collects the data as follows. The total dc bias applied to the load resistor and thermometer is monitored by one channel of the ADC. Another channel monitors the output of the de preamplifier. The computer program calculates the average resistance of the thermometer from these two voltages, and then calculates the temperature of the calorimeter. The regulation voltage is then adjusted by the computer so as to reduce the difference between the measured temperature and the specified temperature. Once the temperature of the calorimeter has been within $0.02 \%$ of the specified temperature for 10 lock-in time constants, the computer begins to record the outputs of the two lock-in amplifiers. The heat capacity is given by the following relationship between the measured quantities:

$$
C=\frac{V_{D C} \alpha V_{H}^{2}}{2 \omega R_{H} V_{A C}}
$$

When the signals have been averaged sufficiently, a new temperature is selected and the process begins again. This continues until the temperature scan is completed. Typically, a 
40-point scan takes less than $1 / 2$ hour to complete. This is important, as the experiments are limited to 8 hours by the hold-time of the cryostat. 


\section{Constraints of the method}

For all of the systems of interest, the lack of an independent measurement of coverage imposes a severe limitation. Desorption measurements in this apparatus are difficult because, after the dose, background pressures of the adsorbed atom are quite high. Signals due to desorbed atoms are difficult to separate from the drift in background pressure. A microbalance-type measurement of the adsorbed mass could be combined with the heat capacity measurement through the use of a piezoelectric substrate and some modifications in the arrangement of the electrical contacts and support assembly.

The small ratio of surface are on the calorimeter to vacuum system volume makes adsorption isotherm measurements impossible. These measurements have greatly aided in the location of phase boundaries of adsorbates on porous substrates.

Diffraction measurements are sometimes used to establish adsorbate coverage. Such techniques require exposure of the substrate to a large solid angle of roomtemperature apparatus. The infrared radiation from room temperature would prohibit operation of these calorimeters at the lowest temperatures, where the physics is the most interesting. Also, photon-stimulated desorption would contribute to the instability of the coverages.

The technique can be extended to lower temperatures without sacrifice in sensitivity. In fact, as the heat capacity of the substrate becomes smaller, the surface sensitivity increases. However, at temperatures below $100 \mathrm{mK}$, difficulties with internal ime constants and electrical nonlinearities may become severe, requiring the development of another thermometer technology. 


\section{References}

1 A.E. Lange et al., Int. J. IR+MM Waves 4, 689 (1983).

2 MSTF-3-S-N-1K, Mini-Systems, Inc., North Attleboro, MA.

3 Epo-Tek H20E, Epoxy Technology Inc., Billerica, Ma.

4 E.E.Haller, IR Phys. 25, 257 (1985).

5 T.W. Kenny, et. al., Phys. Rev. B 39, 8476 (1989).

6 D. McCammon et al,J. Appl. Phys 56, 1263 (1985).

7 M. T. Loponen et al, Phys. Rev. B 25, 1161 (1982).

8 Stychast 2850-FT, Emerson \& Cuming, Inc., Canton, Mass 02021.

9 W.R. McGrath and P.L. Richards, Rev. Sci. Instr. 53, 709 (1982).

10 PAR 5208, Princeton Applied Research Inc., Princeton, NJ.

11 PAR 113, Princeton Applied Research Inc., Princeton, NJ.

12 PAR 5101, Princeton Applied Research Inc., Princeton, NJ.

13 DT2785-DI/5716-B-PGL, Data Translation Inc., Marlboro, MA. 


\section{Measurements}

\section{Baseline}

The heat capacity of the bare calorimeter is measured during each experiment before dosing. The calorimeter is composed of a combination of metals and insulators. Consequently, the low-temperature heat capacity of the calorimeter is expected to be of the form

$$
C(\mathrm{~T})=\mathrm{AT}+\mathrm{BT}^{3}
$$

where the linear term comes from the electron: in the metallic components, and the cubic term comes from the phonons in all of the cornponents. In the temperature range investigated, both terms are expected to contribute to the heat capacity. This form of the heat capacity can be verified by plotting $C / T$ vs $T^{2}$, as in Fig. 10 . The heat capacity of the bare calorimeter should fit a straight line when plotted in this manner. If not, problems with the choice of operating frequency, or complications with internal time constants are the likely cause of the deviation. The slight deviations from linearity shown in the figure result from errors in the calibration of the thermometers at the highest temperatures.

\section{Indium samples}

In order to demonstrate the performance of this apparatus, measurements of the heat capacity of small samples of In were carried out.

The calorimeters are often assembled with a small $(\sim 25 \mu \mathrm{g})$ Indium sample attached to the back surface. These samples are prepared by trimming the edge of a 0.010 in foil with a razor blade. They are weighed in a laboratory microbalance with a resolution of 


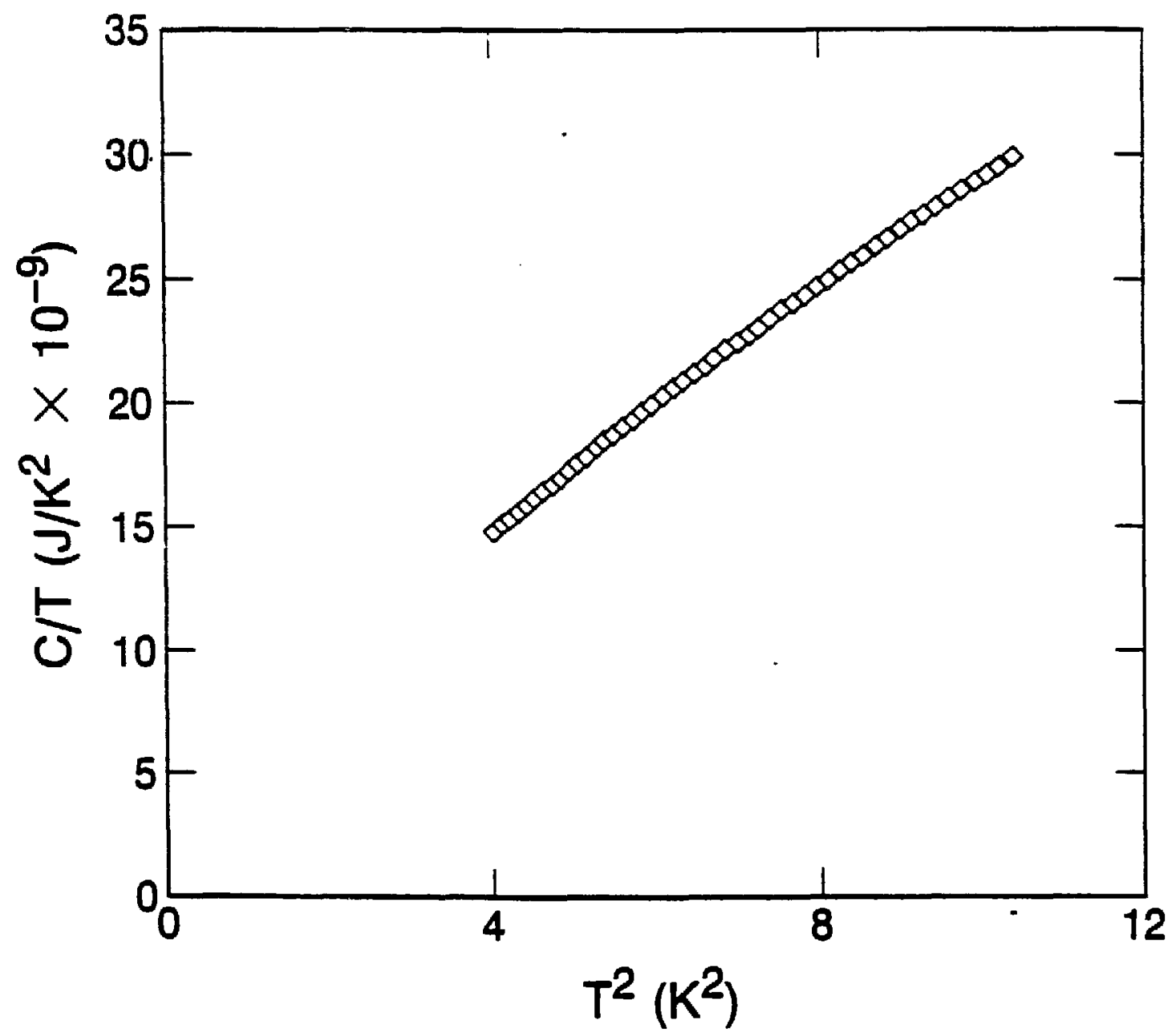

X9L. 893.5068

Figure 10 Heat capacity of the bare calorimeter plotted as $C / T$ vs $\mathrm{T}^{2}$. The slight deviations from linearity are due to errors in the calibration of the thermoneter at the highest temperatures. 
$5 \mu \mathrm{g}$ and then attached to the calorimeter with conductive epoxy. Because of the superconducting phase transition in In, there will be a small discontinuity in the heat capacity of the calorimeter at $3.41 \mathrm{~K}$. The location and magnitude of the discontinuity is routinely studied in the early stages of the characterization of a new calorimeter to verify the calibration of the thermometers and the operation of the instrument.

\section{Expected hysteresis}

Hysteresis in the transitions of type I superconductors has been attributed to supercooling below the superconducting transition.1 There is a positive free energy associated with the creation of boundaries between normal and superconducting phases in the presence of an applied magnetic field. As a result, the normal phase can persist to a temperature below $\mathrm{T}_{\mathrm{c}}$.

Ordinarily, supercooling experiments are carried out by fixing the temperature, applying a magnetic field in excess of the critical field, $\mathrm{H}_{c}(\mathrm{~T})$, and then slowly reducing the field until the superconducting transition occurs at $\mathrm{H}_{\text {appl. }}$. In experiments of this type on single crystals of In near $\mathrm{T}_{c}$, the limiting value of $\mathrm{H}_{\text {appl }}$ was found to be 2

$$
\frac{\mathrm{H}_{\mathrm{appl}}}{\mathrm{H}_{\mathrm{c}}(\mathrm{T})}=0.158
$$

In our experiments, the temperature is varied in the presence of a fixed applied magnetic field which is due to the magnetic field of the earth and the ion pumps. Magnetometer measurements at several positions around and inside the apparatus indicate an ambient magnetic field of about 0.44 Gauss. The greatest amount of supercooling expected in the presence of such a field is given by: 


$$
\Delta \mathrm{T}_{\mathrm{c}}=\mathrm{T}_{\mathrm{c}}\left(1-\left[1-\frac{\mathrm{H}_{\mathrm{apol}}}{0.158 \mathrm{H}_{\mathrm{c}}(0)}\right]^{1 / 2}\right)
$$

The expected upper limit to the supercooling $\Delta \mathrm{T}_{\mathrm{c}}$ of In is about $17 \mathrm{mK}$. The energy required to form superconducting-normal boundaries near defects in the crystal can be much less than in a perfect crystal. Such defects can give rise to the formation of boundaries which trigger the phase transition for smaller values of $\Delta T_{c}$.

\section{Experimental results}

Figure 11 shows the heat capacity of the calorimeter in the neighborhood of the expected superconducting transition of In. Measurements made with both increasing and decreasing temperatures are shown. The observed hysteresis indicates a supercooling of about $4 \mathrm{mK}$, which is well within the limits of ideal supercooling in the presence of the ambient magnetic field. The amplitude of the discontinuity is consistent with that expected for the $25 \pm 5 \mu g$ of In that was attached to this calorimeter.

The sharp discontinuities in Fig. 11 occur because of the hysteresis in the phase transition. In the absence of hysteresis, the transitions would be broadened by the $1 \mathrm{mK}$ temperature oscillation used in the measurement. Figure 12 shows the heat capacity of another calorimeter with a different In sample.In this case, the hysteresis is absent, and the width of the discontinuity is about $5 \mathrm{mK}$, which is well in excess of the $1 \mathrm{mK}$ temperature oscillation. The absence of supercooling and the occurrence of a broadened transition in this sample are probably due to a greater concentration of defects which can nucleate the formation of the superconducting phase. The two In samples investigated here probably differ in the amount and type of crystalline defects, although they were prepared and mounted in a similar manner. 


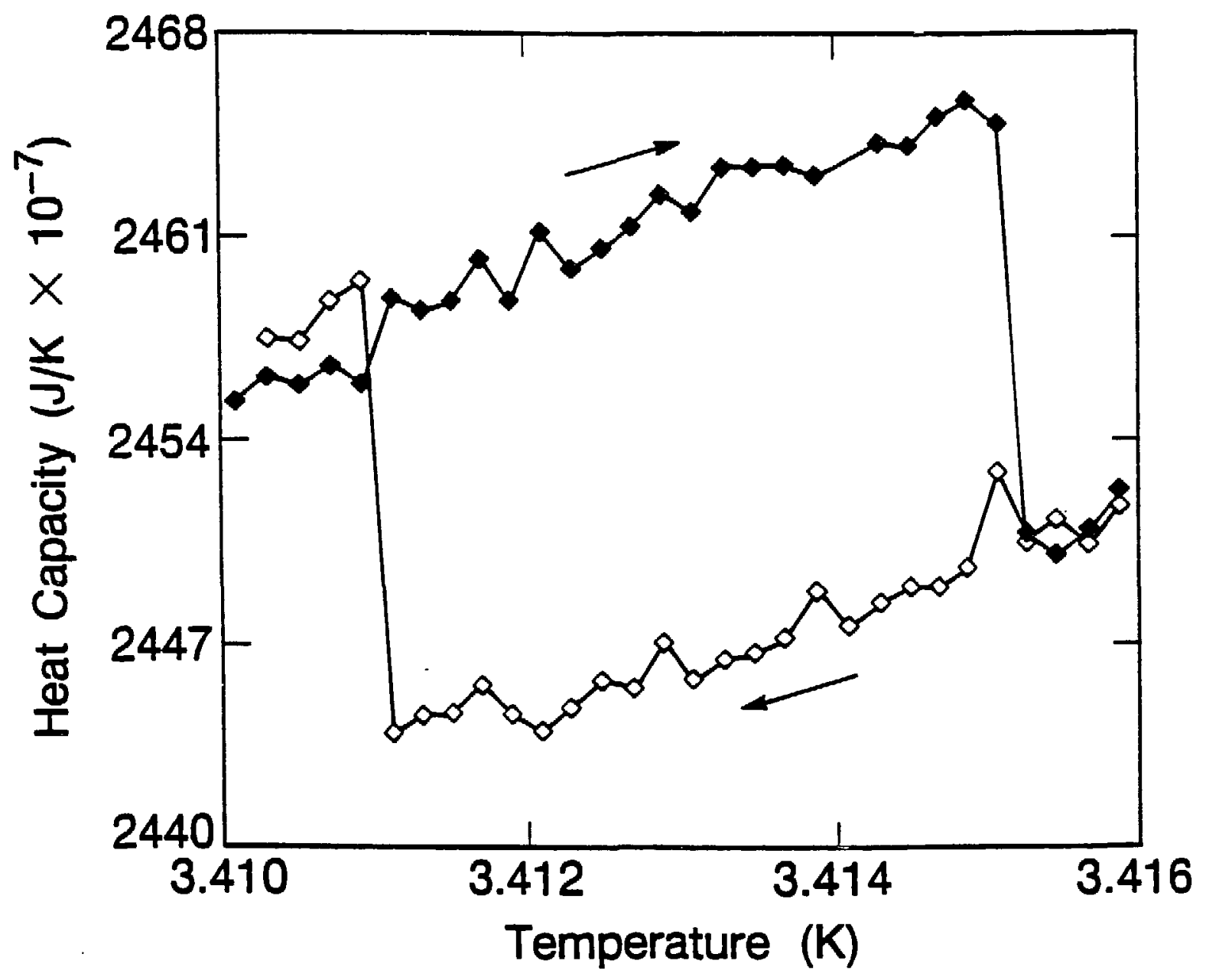

XBL 8910-7633

Figure 11 The hysteresis in the superconducting transition of a $25 \mu \mathrm{g}$ In sample on the calorimeter. Such hysteresis is typical of the expected supercooling in the presence of a small applied magnetic field. 


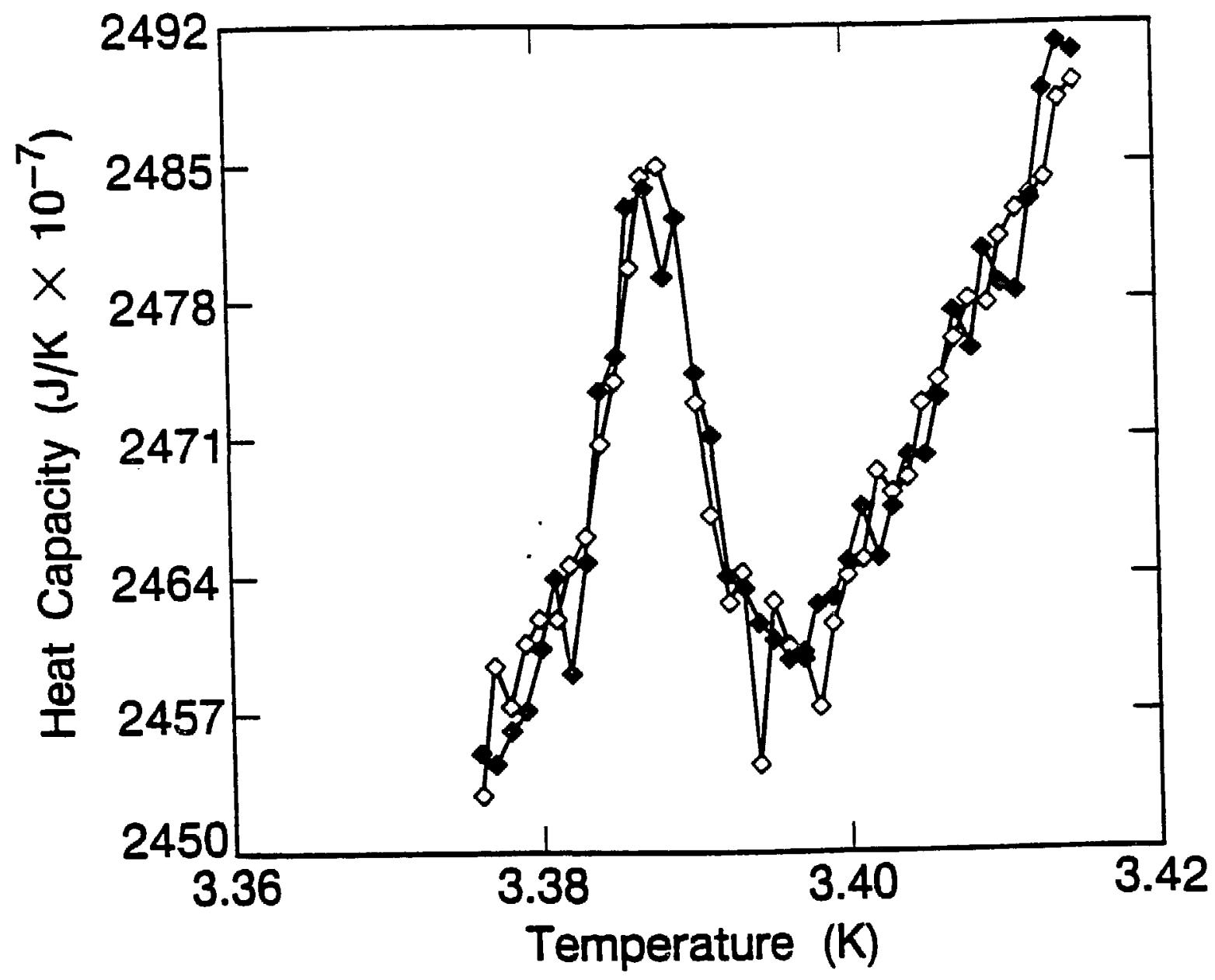

XBL $8810-7634$

Figure 12 The hear capaciry of a second In sample attached in a similar manner to that in Fig. 11. In this case, supercooling is not observed, probably because of a greater concentration of defects which can nucleate the formation of the superconducting phase. 
The sensitivity of the measurement as illustrated in Figs. 11 and 12 is of order 10-10 $\mathrm{J} / \mathrm{K}$ at $3.4 \mathrm{~K}$ for a temperature oscillation of $-1 \mathrm{mK}$. For comparison, the heat capacity of 0.1 monolayer of adsorbed ${ }^{4} \mathrm{He}$ in the gas phase on one surface of the calorimeter is of order $5 \times 10^{-10} \mathrm{~J} / \mathrm{K}$.

\section{He adsorbed on sapphire}

Before exploring the heat capacity of adsorbates on metallic films deposited on the front surface of the calorimeter, we undertook a study of the heat capacity of submonolayers of ${ }^{4} \mathrm{He}$ adsorbed on bare, optically polished sapphire. This study is of practical importance to the operation of composite infrared bolometers that are similar in construction to this calorimeter. The added heat capacity of one adsorbed monolayer on such a bolometer has never been measured directly, although reductions in bolometer sensitivity have been observed upon exposure to small amounts of ${ }^{4} \mathrm{He} .{ }^{3}$

The sapphire substrates were ultrasonically cleaned in electronic grade $1,1,1-$ trichloroethelyene, acetone and methanol prior to assembly of the calorimeter. After this trearment, the substrates were handled only on the edges with stainless steel tweezers that were cleaned in the same manner. Bismuth films were deposited on the back surface as resistive heaters, and the substrates were then installed in an aluminum frame. Doped germanium thermometers and copper wires were attached to the back of the substrate with conductive epoxy. ${ }^{4}$ The entire assembly was baked in air at $100 \mathrm{C}$ for several hours to complete curing of the epoxy. The calorimeters were submerged in ${ }^{4} \mathrm{He}$ for calibration of the thermometers and then installed in the UHV system. After several days of pumping without bakeout, the base pressure of this system is as low as 10-9 torr. Upon cooling of the radiation shields to $100 \mathrm{~K}$ and the cold finger to $1.5 \mathrm{~K}$, the pressure near the calorimeter is less than $10^{-11}$ torr. Before measurements the calorimeter was briefly warmed to slightly above room temperature to remove adsorbed contamination. This temperarure is limited by 
the degradation of the epoxy used to fabricate the calorimeter. No further surface preparation was used for these studies. It is probable that water was present on the surface. Adsorbed hydrocarbons were also possible.

At the beginning of each experiment, the heat capacity of the bare calorimeter was measured at a series of temperatures between 1.7 and $4.2 \mathrm{~K}$. Then the front surface of the calorimeter was exposed to a beam of ${ }^{4} \mathrm{He}$ atoms from a room temperanure effusion cell. The pressure in the effusion cell was monitored with an ionization gauge. Since the geometry of the cell and the distance to the calorimeter are known, the number of incident atoms per unit time is known. Exposures of 5 atoms per square angstrom of sapphire were typical. However, an undetermined fraction of the incident ${ }^{4} \mathrm{He}$ atoms adsorb on the sapphire under these circumstances, so the absolute coverage is unknown. After the exposure was completed, the heat capacity of the calorimeter plus adsorbates was measured at the same temperatures as before the dose. The heat capacity of the ${ }^{4} \mathrm{He}$ is obtained by subtraction.

The heat capacity of several coverages of ${ }^{4} \mathrm{He}$ on sapphire was measured. The data for three of these coverages are shown in Fig. 13. Since the absolute coverage is unknown, the units on the vertical axis are simply $\mathrm{J} / \mathrm{K}$ for the adsorbed ${ }^{4} \mathrm{He}$, which is spread over the $0.32 \mathrm{~cm}^{2}$ surface area of the front of the calorimeter below $3.3 \mathrm{~K}$. Above $3.3 \mathrm{~K}$, the heat capacity of the adsorbed ${ }^{4} \mathrm{He}$ decreases with increasing temperature. Measurements of the heat capacity at $2 \mathrm{~K}$ separated by brief ramps to higher temperatures demonstrated that desorption occurs above $3.3 \mathrm{~K}$. The ${ }^{4} \mathrm{He}$ is entirely desorbed from the sapphire at $4.2 \mathrm{~K}$. The measured heat capacity below $3.3 \mathrm{~K}$ can be interpreted as a superposition of two components. One component has heat capacity independent of coverage. The heat capacity of this component increases with temperature from 1.8 to 3.3 $\mathrm{K}$. The second component is independent of temperarure but increases with coverage. 


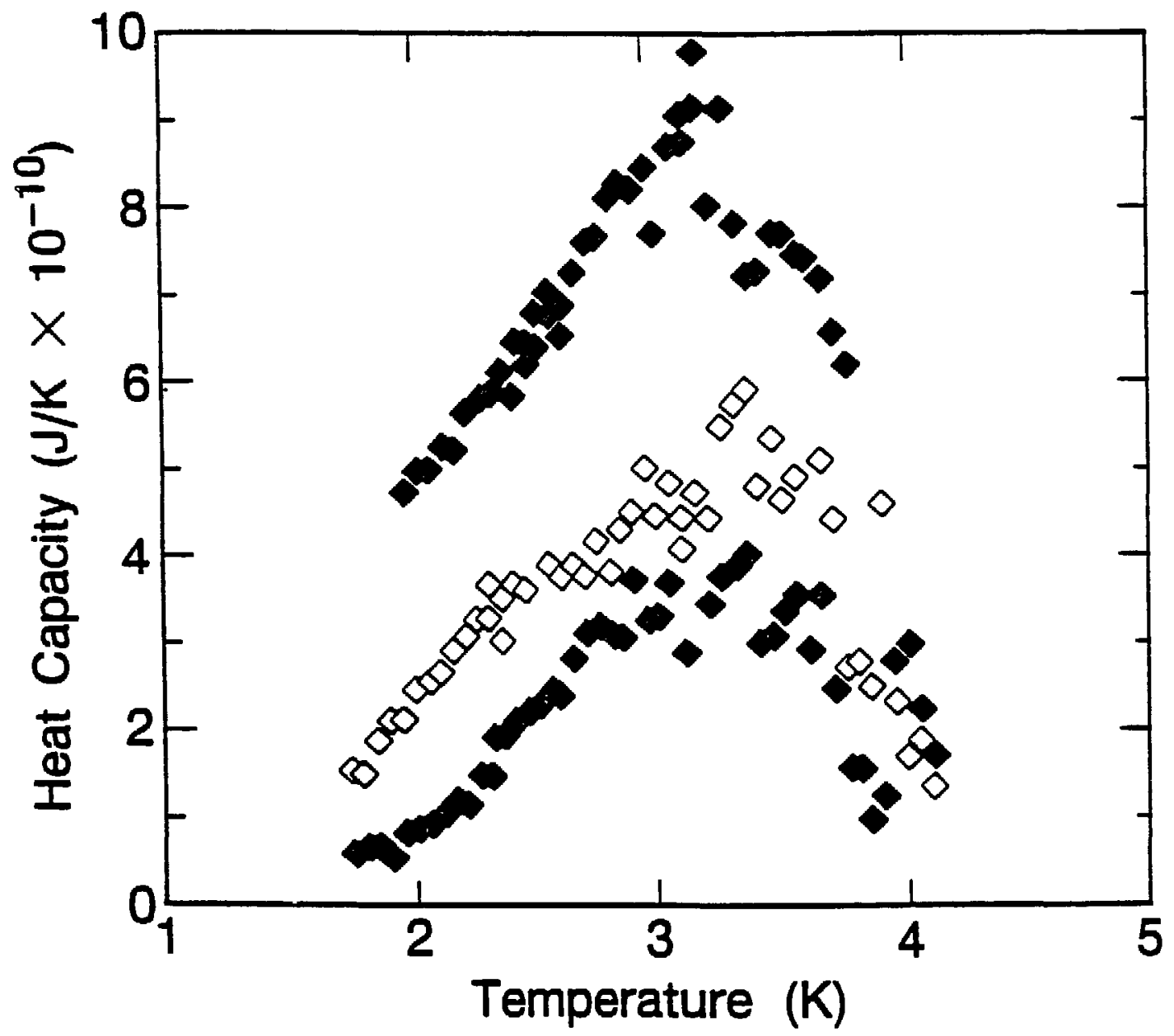

XEL $8810-7666$

Figure 13 The hear capacity of three coverages of ${ }^{4} \mathrm{He}$ on sapphire. The heat capacity of the bare calorimeter has already been subtracted. The temperature dependence above 3.3 $\mathrm{K}$ is dominated by desorption of the adsorbed ${ }^{4} \mathrm{He}$. 
A qualitative understanding of these results is possible despite the lack of a well characterized substrate. The ${ }^{4} \mathrm{He}$ states with the lowest free energy are laterally bound near defects and impurities on the sapphire surface. Higher energy states with lateral mobility exist over the rest of the surface. A low coverage of ${ }^{4} \mathrm{He}$ would occupy the lowest energy bound states first. Higher coverages of ${ }^{4} \mathrm{He}$ would occupy some mobile states as well as the bound states. The heat capacity of a low coverage of ${ }^{4} \mathrm{He}$ would increase with temperature due to excitation of atoms within bound states and promotion from bound states to mobile states. The heat capacity of a high coverage of ${ }^{4} \mathrm{He}$ would have the same contribution from the bound states as the low coverage, plus an additional temperatureindependent component due to the mobile states. The sudden decreases in heat capacity above $3.3 \mathrm{~K}$ occur due to desorption of the ${ }^{4} \mathrm{He}$.

A more detailed explanation of these results would require knowledge of the distribution of binding energies presented by a partially contaminated sapphire surface. Rather than continue studies of ${ }^{4} \mathrm{He}$ on sapphire, we have carried out studies of ${ }^{4} \mathrm{He}$ on evaporated noble metal films. Preliminary measurements of ${ }^{4} \mathrm{He}$ adsorbed on evaporated Ag films in this apparatus have seen evidence for a gas phase over a wide range of coverages. 5

\section{CO adsorbed on $\mathrm{Ag}$}

Monolayers of $\mathrm{CO}$ adsorbed on Ag films have been widely studied due to the observation of large enhancements in Raman signals on cold-condensed films. This cryostat was previously used for an investigation of the infrared absorption of $\mathrm{CO}$ monolayers as a function of the substrate condensation temperature, coverage, and other variables. 
Below $4 \mathrm{~K}$, the heat capacity of a monolayer of solid $\mathrm{CO}$ should be proportional to $\mathrm{T}^{2}$ and several orders of magnitude smaller than a monolayer of ${ }^{4} \mathrm{He}$. This is because the only excitations available to the $\mathrm{CO}$ are long wavelength phonons, and the density of states associated with these excitations goes to zero at low energy. However, the heat capaciry of mulilayers of $\mathrm{CO}$ is detectable. We have carried out a series of measurements of the heat capacity of $\mathrm{CO}$ multilayers adsorbed on room-temperature evaporated $\mathrm{Ag}$ films in order to investigate the variations which occur as the multilayers become macroscopically thick.

\section{Measurements}

Multilayers of $\mathrm{CO}$ were deposited on the front surface of the calorimeter while the temperature of the calorimeter was maintained at $\sim 2.5 \mathrm{~K}$. Under these circumstances, the $\mathrm{CO}$ molecules become physisorbed with probability very close to 1 . Therefore, the $\mathrm{CO}$ coverage after the dose is complete is equal to the total $\mathrm{CO}$ exposure during the dose.

The temperature dependence of the heat capacity of a series of $\mathrm{CO}$ coverages ranging from 10-80 layers was measured. The measured temperature dependence of the heat capacity for each coverage is fitted to a power law and the exponent which provided the best fit is plotted as a function of the number of $\mathrm{CO}$ layers in Fig. 14. The figure shows that for coverages of less than 20 layers, the heat capacity is proportional to $T^{2}$, while for coverages of more than 50 layers, the heat capacity is proportional to $\mathrm{T}^{3}$. Errors associated with fitting of the temperature dependence of the heat capacity with a power law are indicated by the error bars on the figure.

\section{2-D to 3-D Debve theory}

The heat capacity of a simple two-dimensional insulator can be easily calculated. The density of states for phonons in two-dimensions is given by 


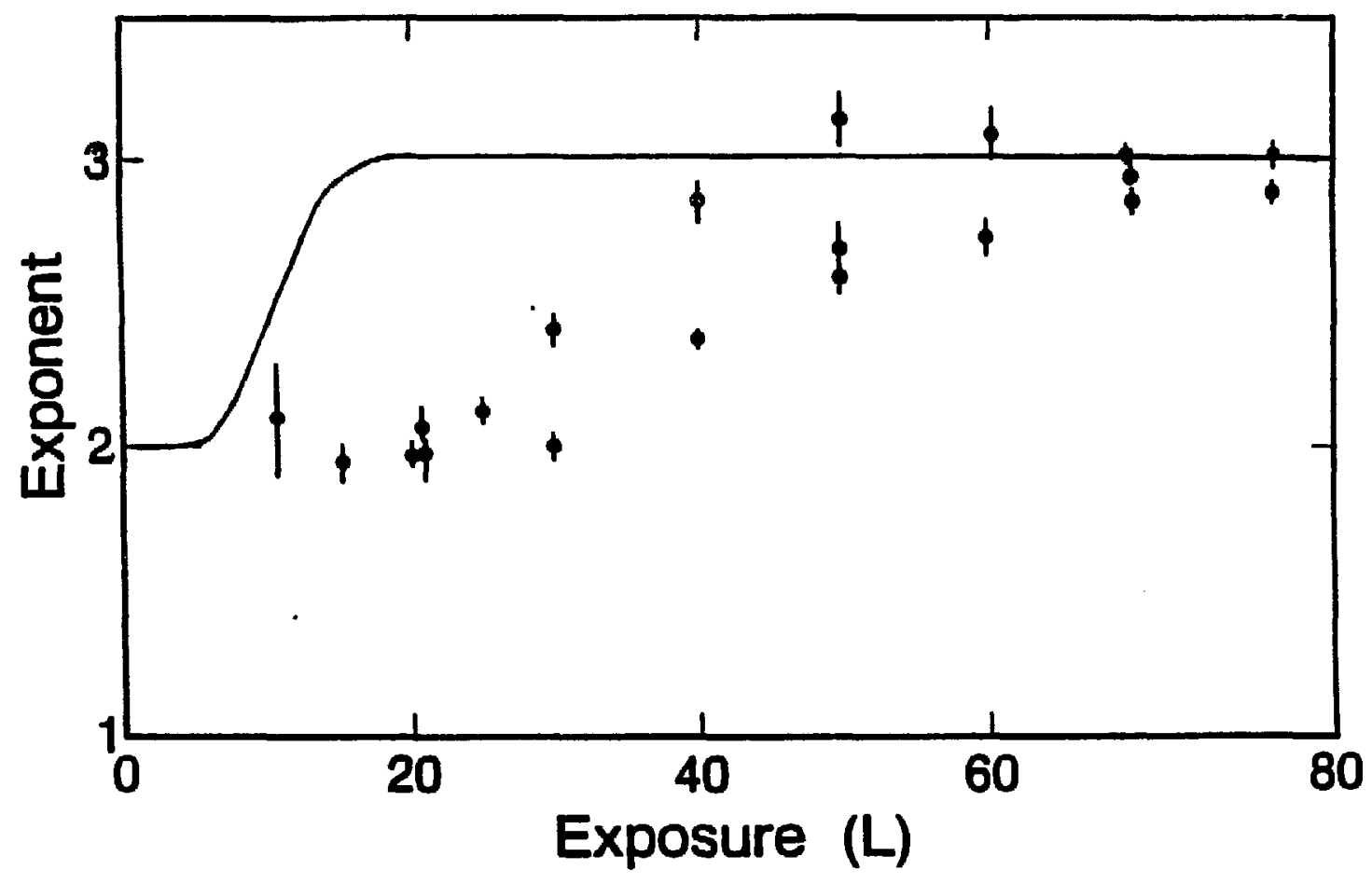

XBL $868-8916$

Figure 14 The exponent of the power law of the temperature dependence of the heat capaciry plotted as a function of the $\mathrm{CO}$ exposure, which is equal to the thickness of the $\mathrm{CO}$ in layers. For thin films, the heat capacity is proportional to $\mathrm{T}^{2}$, and for thick films the heat capacity is proportional to $\mathrm{T}^{3}$. 


$$
D(\omega)=\frac{L^{2} \omega}{2 \pi v^{2}}
$$

where $L^{2}$ is the area of the system and $v$ is the acoustic phonon velocity. This density of states is inserted into the integral for the energy,

$$
E=\frac{L^{2}}{2 \pi v^{2}} \int \frac{w d w}{\exp (h w / k t)+1}
$$

which, upon differentiation with respect to $T$ yields

$$
\mathrm{C}=(28.8) \mathrm{N} \mathrm{kB}(\mathrm{T} / \theta)^{2}, \text { where } \theta=\frac{h \mathrm{v}}{\pi \mathrm{kB}_{B}} \sqrt{\frac{\pi \mathrm{N}}{\mathrm{L}^{2}}}
$$

For multilayer systems, phonons can propagate in the third dimension. In phase space, these additional phonon modes can be represented as additional sheets of states which are offset vertically from $k_{z}=0$. The arrangement of phonon modes in $k$-space for such a multilayer system is schematically represented in Fig. 15. The density of states for such a system differs from the two-dimensional case at energies above that of the lowest energy phonon propagating in the third dimension. At these energies, discontinuities appear in the density of states, which is given by:

$$
D(\omega)=\frac{L^{2} \omega}{2 \pi v^{2}}+\sum_{i=1}^{\omega t / 2 \pi v} 2 i
$$

where $t=$ the total thickness of the multilayer system. Another way of describing this is to note that the wavcier, gth of the lowest energy phonon available in the third dimension is 


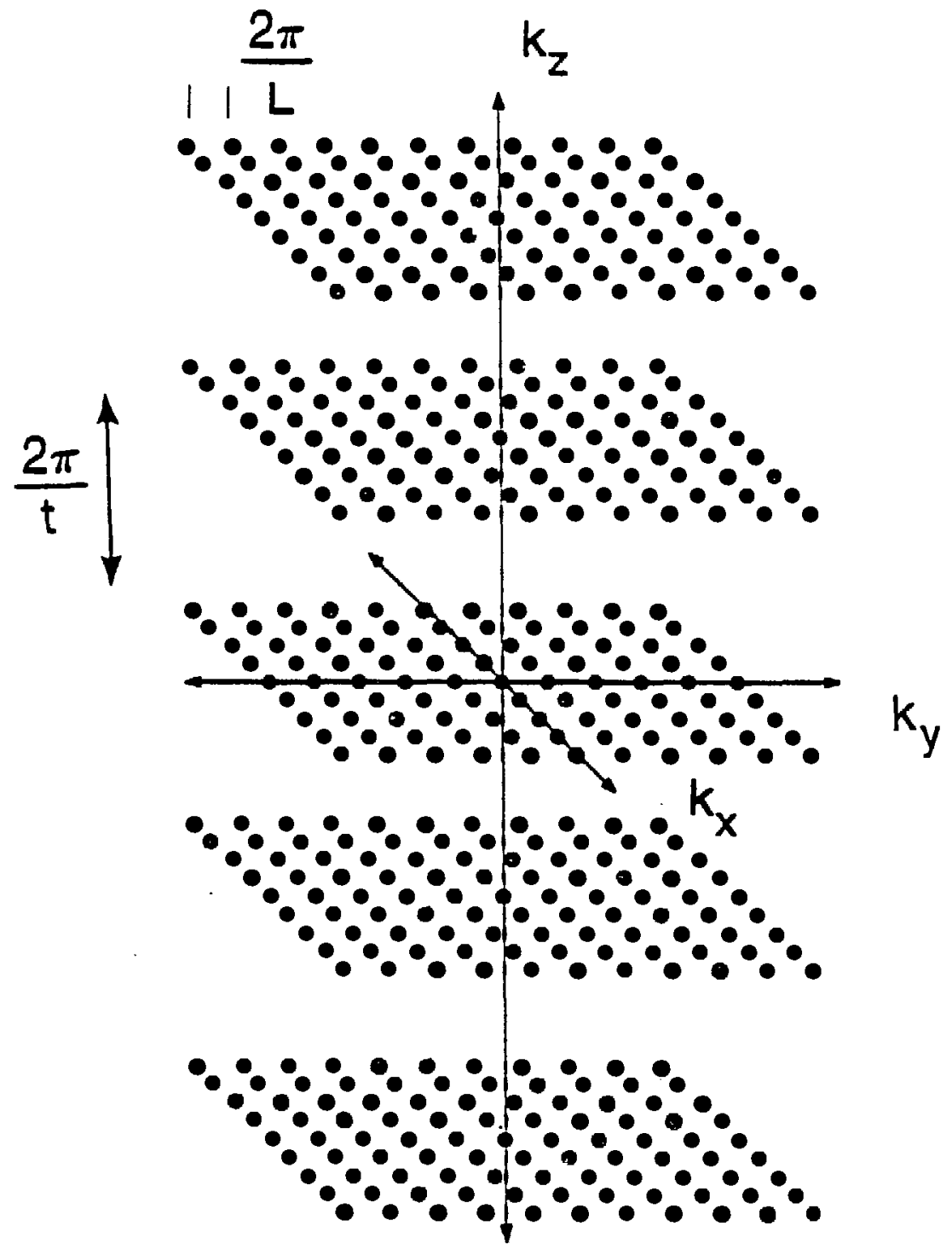

Figure 15 Schematic drawing of the arrangement of the phonon modes for a thin, multilayer system in $k$-space. the lateral spacing between modes is $2 \pi / \mathrm{L}$, whereas the vertical spacing is $2 \pi / t$, where $L$ is the lateral dimension and $t$ is the thickness. 
equal to twice the thickness of the system. For lower energies and longer wavelengths, the system only has phonons propagating in two-dimensions. For higher energies and shorter wavelengths, the third dimension becomes thermally populated as well, and the temperature dependence of the heat capacity exceeds the two-dimensional limit. As the thickness becomes comparable to the lateral dimensions of the system, the spacing between the sheets in k-space approaches the separation between points within the sheets. The muldilayer density of states associated with this distribution of excitarions is shown in Fig. 16 along with the two-dimensional and three-dimensional limiting cases.

Note that, as long as the films are thin and the temperatures are low enough that the phonon modes perpendicular to the surface are not populated, the heat capacity of the multilayer system is expected to be proportional to $T^{2}$ and independent of the number of adsorbed layers. This is because the changes which occur due to the addition of layers to the system only appear in the number of and the spacing between the addirional sheets of states in k-space. As long as these states are not thermally populated, they should not contribute to the heat capacity. Therefore, the prediction of this model is that, for small numbers of layers, the heat capacity should be proportional to $\mathrm{T}^{2}$, and independent of the number of layers, while for many layers, the heat capacity should be proportional to $\mathrm{T}^{3}$ and proportional to the number of layers.

The expression for the energy of the system obtained from the multilayer densiry of states must be integrated numerically, before the temperarure dependence of the heat capacity can be calculated. This calculation has been carried out with parameters appropriate for bulk solid $\mathrm{CO}$, and the resulting temperature dependence of the heat capacity has been again fit to a power law. The exponent which was extracted from these fits is plotted in Fig. 14 along with the experimental results. Note that the experimentally 


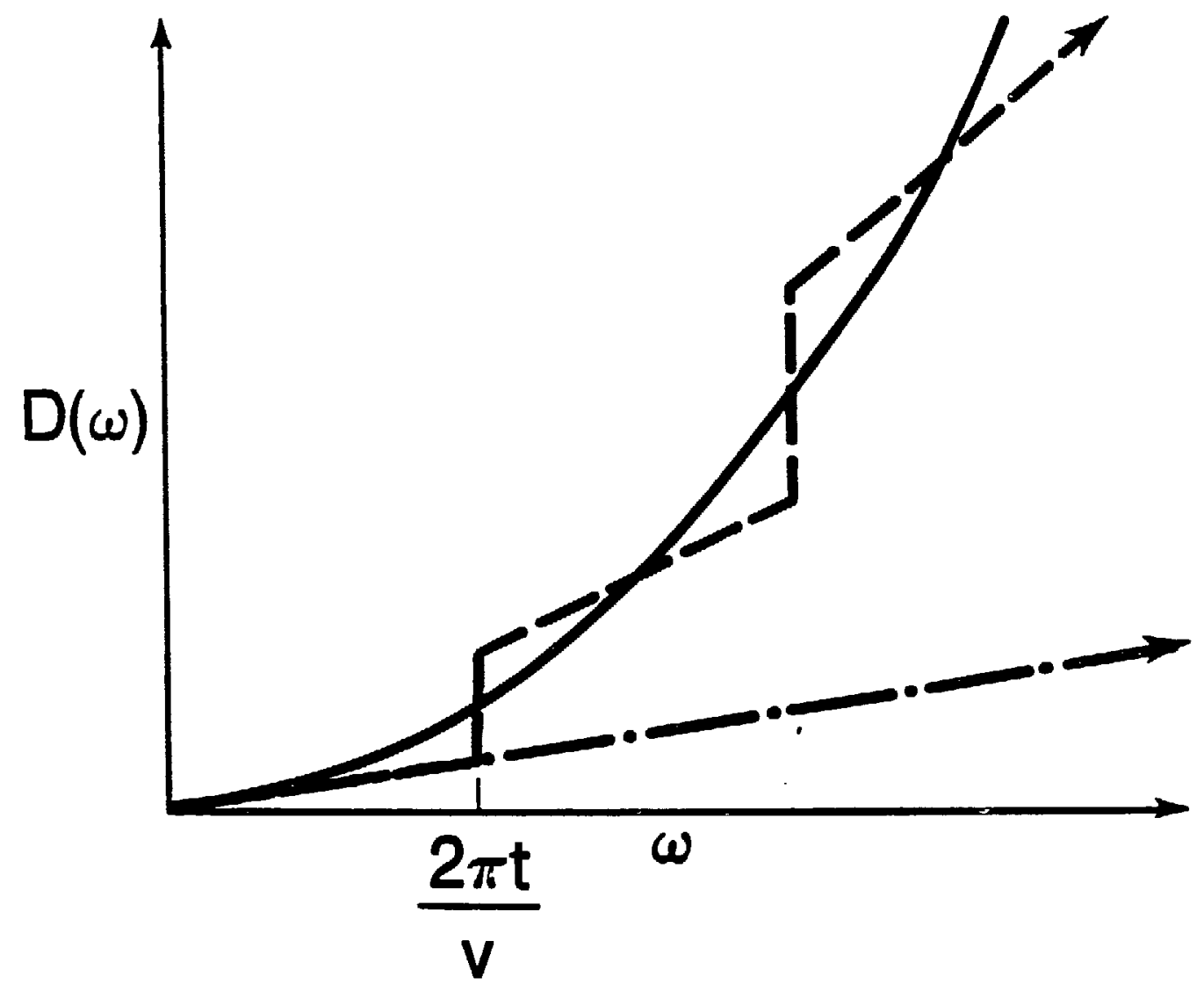

XBL 893-5064

Figure 16 Density of states as a function of energy for 2-dimensional, 3-dimensional, and thin multilayer systems. The locations of the discontinuities for the multilayer system is determined by the thickness of the multilayer. 
observed onset of deviations from $\mathrm{T}^{2}$ occurs at about a factor of 3 higher in coverage than the theoretical prediction.

There is a discrepancy between the locations of the predicted and observed deviations from $\mathrm{T}^{2}$ temperature dependence. In addition, it was found that the heat capacity of the system near $2 \mathrm{~K}$ is proportional to the coverage even when the temperanure dependence of the heat capacity is proportional to $T^{2}$. These two discrepancies suggest that this simple model is inadequate.

\section{More complicated models}

It is expected that the first several layers of $\mathrm{CO}$ should be compressed due to the additional attraction to the $\mathrm{Ag}$ substrate. If so, those layers would have a Debye temperature higher than the assumed bulk value. The average Debye temperature of such a system should gradually approach the bulk Debye temperature from above as the thickness is increased. The heat capacity of such a system would increase with coverage but the temperature dependence would still be proportional to $T^{2}$. In addition, the appearance of thermally excited phonons perpendicular to the surface would be postponed to higher coverages. Given a more complete set of data, it would be possible to extract the coverage dependence of the average Debye temperature.

\section{${ }^{4} \mathrm{He}$ adsorbed on $\mathrm{Ag}$}

In order to fully understand the effects of the graphite surface on the properties of ${ }^{4} \mathrm{He}$ adsorbed on graphite, it is necessary to investigate the properties of ${ }^{4} \mathrm{He}$ monolayers on different substrates. Measurements on Vycor, $\mathrm{MgO}$ smoke, Mylar, and other substrates have been carried out, but have been hampered by the difficulties in the characterization of these substrates. Metaliic 
substrates are expected to present a more uniform physisorption environment due to the nonlocalized nature of the electronic wave functions at the metal surface. 6

Because of the widespread interest in Ag films for their role in surface enhanced Raman experiments, their structural characteristics have been extensively investigated. Scanning tunneling microscopy (STM) has shown films deposited on room-temperature sapphire substrates to be composed of $\mathrm{Ag}$ (111) crystallites of about $500 \AA$ in lateral dimension, separated by compact boundary regions. ${ }^{7}$ Scanning electron microscopy (SEM) of the films used in these studies shows $500 \AA$ crystallites, and STM studies of these films are underway.

\section{Heat Capacity Measurements.}

The measured heat capacity of several coverages of ${ }^{4} \mathrm{He}$ adsorbed on $\mathrm{Ag}$ are shown in Fig. 17 as a function of temperature between 1.7 and $3.3 \mathrm{~K}$. At the lowest coverages invescigated, the heat capacity is independent of the temperature. As the coverage is increased, the heat capacity is seen to decrease with decreasing temperature. The sensitivity of the measurement degrades rapidly with increasing temperature due to an increase in the heat capacity of the substrate and a reduction in the sensitivity of the thermometer. More accurate measurements above $2.5 \mathrm{~K}$ have been made by increasing the amplitude of the temperature oscillation. These measurements confirm that the heat capacity is independent of temperature between 2.8 and $3.3 \mathrm{~K}$ for the two lowest coverages shown. Above $3.3 \mathrm{~K}$, desorption of the ${ }^{4} \mathrm{He}$ occurs. The differences in temperature between the calorimeter and the heat sinks occasionally induce migration of the ${ }^{4} \mathrm{He}$ off of the calorimeter at the highest temperatures of the measurement. The migration usually appears after several cycles of the entire apparatus to room temperature, and is attributed to the gradual adsorption of contaminants on the back surface of the calorimeter. Migration can be distinguished from desorption because of the retum of the adsorbed ${ }^{4} \mathrm{He}$ upon cooling of the calorimeter. The 


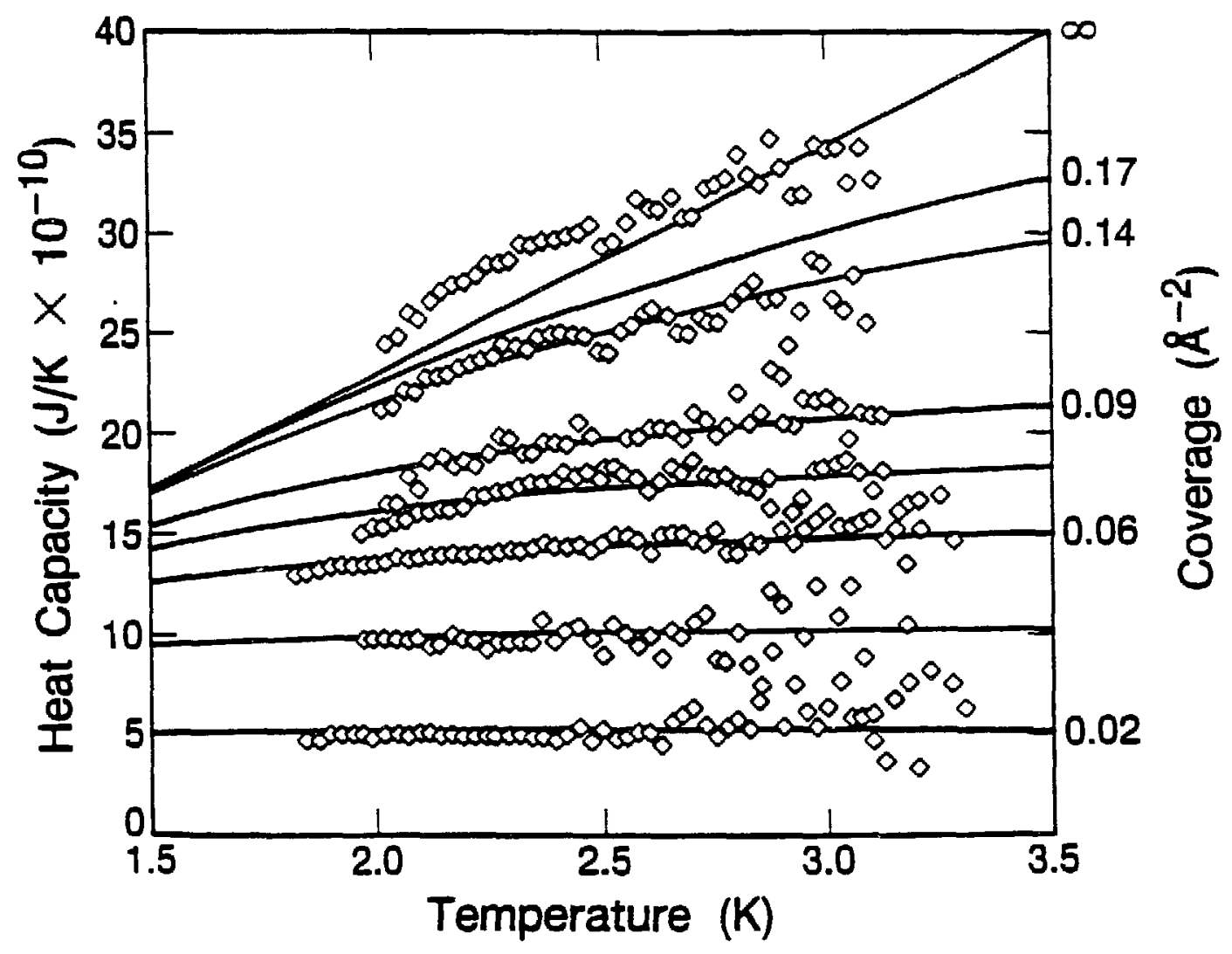

XBL 803.50671

Figure 17 Measurements of the temperature dependence of the heat capacity for a series of coverages of ${ }^{4} \mathrm{He}$ on $\mathrm{Ag}$ films. The solid lines correspond to the calculated heat capacity of a 2-dimensional non-interacting Bose gas. 
results described here have been reproduced with several calorimeters which have not yet shown effects of migration.

There are a number of striking differences between these data and those for similar coverages of ${ }^{4} \mathrm{He}$ on graphite substrates. The absence of Schottky-type anomalies is taken as zvidence that the corrugation in the binding energy which occurs on insulating substrates is considerably weaker for the case of Ag substrates. This smaller corrugation of the binding energy is expected for metal substrates. There is no evidence of the increase in heat capacity with decreasing temperatures predicted from the virial expansion of the adsorbate-adsorbate interaction, or from a melting transition from the hexagonal incommensurate phase that is expected at low temperatures.8

There are reasons to expect that the adsorbate-adsorbate interaction will be reduced by the electrons in the substrate. The image dipoles which appear below the metal surface act to partially cancel the second order attraction due to correlated dipole fluctuations, and the electrons near the metal surface induce static dipole moments, which contribute a slight repulsion. ${ }^{9}$ A number of procedures for estimating these effects are published 10,11 . At the average separations typical of the highest coverages investigated here, however, these corrections reduce the adsorbate-adsorbate interactions by less than $5 \%$.

The ${ }^{4} \mathrm{He}-\mathrm{Ag}$ attraction is measured to be approximately one fourth as strong as the ${ }^{4} \mathrm{He}$-graphite attraction ${ }^{12,13}$. It is possible that the $\mathrm{Ag}$ surface does not constrain the adsorbed monolayer as tightly, allowing the gas phase to persist to higher coverages and lower temperatures than on graphite. The classical turning points for the ground state in the normal direction for adsorbed ${ }^{4} \mathrm{He}$ are $40 \%$ further apart on $\mathrm{Ag}$ than on graphite. This leads to a $10 \%$ increase in the number of spheres with the classical radius of ${ }^{4} \mathrm{He}$ that could be packed into a single layer. The only precise theoretical descriptions of thermodynamics 
in two dimensions which address the normal component of the adsorbate-substrate interaction are those which include the excited states in addition to the ground state of that attraction9. This contribution leads to a rapid rise in the heat capacity with increasing temperature. For ${ }^{4} \mathrm{He}$ on graphite, the excited states are expected to contribute to the heat capacity at temperatures above $6 \mathrm{~K}$. The first excited state for ${ }^{4} \mathrm{He}$ on $\mathrm{Ag}$ is calculated to be $1.9 \mathrm{meV}$ above the ground state, compared with $5.7 \mathrm{meV}$ for graphite, so this contribution should appear at temperatures above $2 \mathrm{~K}$. We see no evidence of this contribution at temperatures up to $3.3 \mathrm{~K}$. Theoretical descriptions of solidification in two-dimensions do not include the strength of the attraction to the substrate, and the importance of this attraction for the formation of the two-dimensional solids is unclear.

Since the signatures of adsorbate-adsorbate or adsorbate-substrate interactions do not appear in the data, we compare the results with the heat capacity of two-dimensional non-interacting bose gases. 14 The energy of the system can be written

$$
U(t)=\frac{2 \pi m A}{h^{2}} \int_{0}^{\infty} \frac{E d E}{e^{[\beta(E-\mu)]}-1},
$$

where $\mathrm{A}$ is the area of the two-dimensional system, and $\mu$ is the chemical potential. Forcing that number of bosons in the system to be a constant yields an expression for the chemical potential,

$$
\mu=k_{B} T \ln \left[1-e^{-N h^{2} / 2 \pi m A k_{B} T}\right] .
$$

The chemical potential approaches zero at low temperatures. In this limit, the expression for the energy (Eq. 17) can be evaluated exactly, and then differentiated to give 


$$
C(T)=\frac{\pi^{3} k_{\mathrm{p}} \mathrm{mA}}{3 h^{2}} T
$$

which is independent of the number of bosons in the system. At such low temperatures, nearly all of the bosons are in the ground state, where they do not contribute to the heat capacity. At higher temperatures, the restriction on the number of bosons in excited states due to finite coverage becomes important, and the chemical potential takes on a finite value. As the temperatures are increased, the heat capacity eventually approaches the limit given by classical equipartition

$$
\mathrm{C}(\mathrm{T})=\mathrm{N} \mathrm{k}_{\mathrm{B}} \text {. }
$$

The expression for the energy of the two-dimensional bose gas has been numerically integrated, and then differentiated to give the heat capacity as a function of temperature for several coverages. These results are shown as smooth curves in Fig. 17 for coverages selected to fit the data. The measured deviations from the equipartition limit which occur at lower temperatures are in excellent agreement with the predictions of this simple theory even at coverages in excess of 0.5 monolayers, as defined in terms of monolayer coverages on graphite. At higher coverages, the measured heat capacity is larger than the theoretical prediction, but the effects of adsorbate-adsorbate interactions, which are ignored in this model, may be important.

\section{Sricking Coefficient Measurements}

In order to obtain a measurement of the sticking coefficient, the change in heat capacity can be monitored while the sample was dosed at several constant temperatures. A 
plot of such data as a function of the exposure calculated from the properties of the effusion cell is shown for three substrate temperatures in Fig. 18. At temperatures above $2.5 \mathrm{~K}$ and heat capacities below $20 \times 10^{-10} \mathrm{~J} / \mathrm{K}$, the heat capacity is a measure of the number of adsorbed atoms. The coverages estimated in this manner are shown on the right vertical axis of Fig. 18. The derivative of the coverage with respect to the exposure is defined as the sticking probability. At the lowest coverages, the sticking probability is equal to $1.8 \%$, independent of coverage or substrate temperature. At higher coverages, the sticking probability abruptly decteases to about $0.8 \%$, at coverages less than 0.5 monolayers. The coverage at which the sticking probability changes decreases with increasing substrate temperatures.

The calculation of the ${ }^{4} \mathrm{He}$ coverage from the heat capacity measurement relies upon the knowledge of the surface area of the $\mathrm{Ag}$ film. It is deposited through a mask whose area is known to within 5\%. Because of the roughness of the Ag film, the surface area may be somewhat larger than that of the opening in the mask. If the STM measurement ${ }^{16}$ of the structure also applies to our films, the area is enhanced by the roughness by only $1 \%$, and this correction to the area is neglected.

\section{Discussion}

The measurements of the sticking probability for room temperature ${ }^{4} \mathrm{He}$ atoms on cold $\mathrm{Ag}$ have a number of interesting features. As shown in the inset in Fig. 18, the sticking probability is independent of substrate temperature at very low coverages. If the adsorbate energy is initially dissipated through the creation of a small number of substrate phonons with energies much larger than $\mathrm{k}_{\mathrm{B}} \mathrm{T}$ for the substrate, only spontaneous emission of phonons occurs, and this process should be independent of subst : :mperature. 


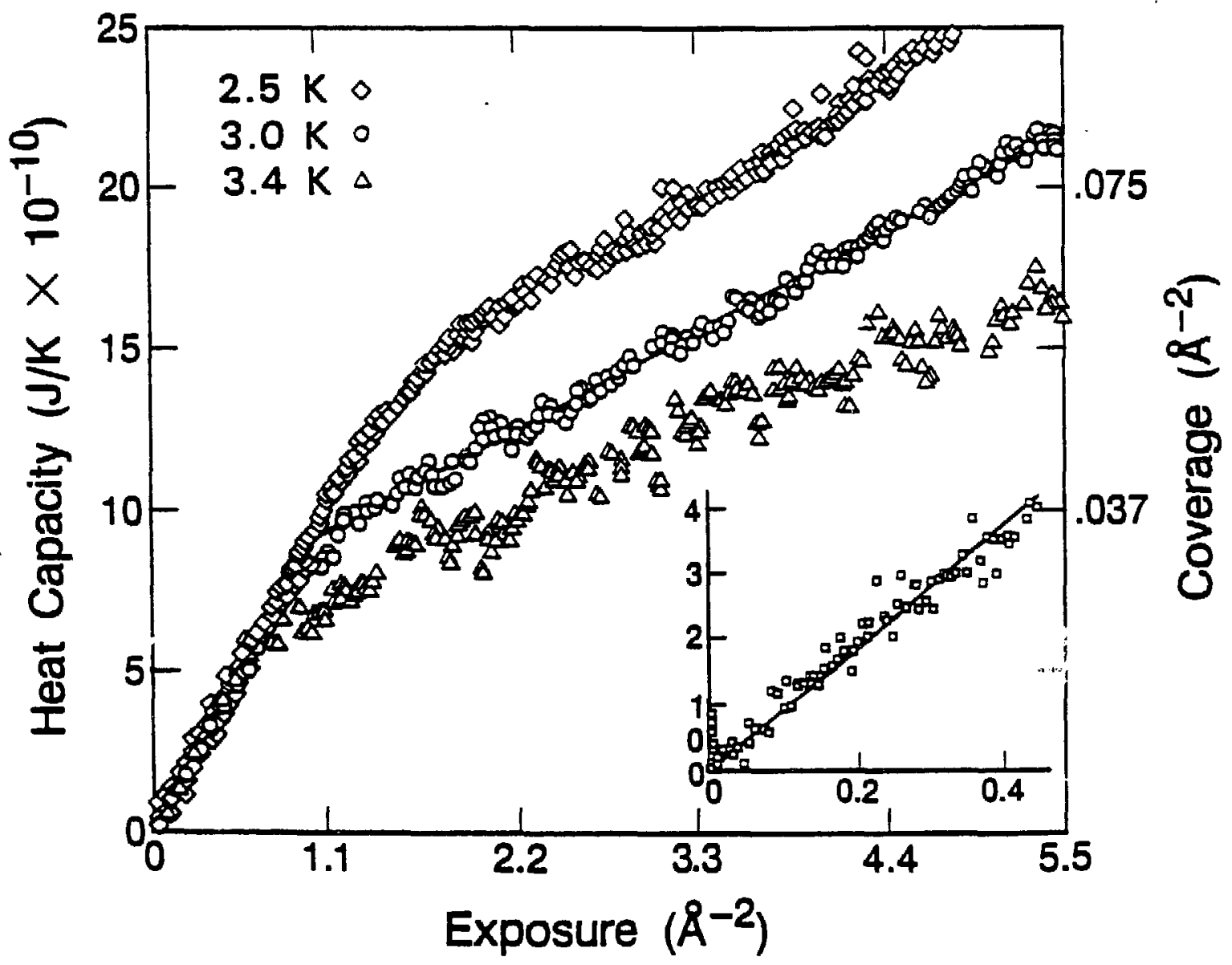

$X E L \operatorname{003} 5000$

Figure 18 Measurements of the number of adsorbed atoms as a function of the number of incident atoms for ${ }^{4} \mathrm{He}$ on $\mathrm{Ag}$ at various substrate temperatures. The slope of the curves shown here is the sticking probability. The lowest coverage data is expanded in the inset. 
Defects or impurities on the Ag surface with greater binding energy than the rest of the surface would be expected to freeze out the first adsorbed atoms. In the measurement of the sticking coefficient, such effects would appear as a non-zero $\mathrm{x}$-intercept corresponding to the adsorption of the first atoms in states with low heat capacity. The measured $\mathrm{x}$-intercept is indistinguishable from zero for coverages of less than $1 \%$ of a monolayer. The occurrance of the ideal gas phase at such low coverages is strong evidence that the $\mathrm{Ag}$ film is highly uniform for ${ }^{4} \mathrm{He}$ physisorption.

The appearance of abrupt changes in the measured sticking probability as a function of coverage usually implies either solidification in the adsorbed layer, or layer completion and promotion into the second layer. Near such structural changes, there are corresponding changes in the entropy of the adsorbate, which should appear as large anomalies in the temperature dependence of the heat capacity. The absence of such features in the measured temperature dependence of the heat capacity nules out these explanations for the changes in the sticking probability. Any explanations that would depend on inhomogeneities in the binding energy are contradicted by the extreme uniformity of the substrate as demonstrated by the appearance of the gas phase at very low coverages. Adsorption processes which involve precursor adsorption into excited states and then relaxation into the ground states should be independent of temperatures at these temperatures. We have no model for this complicated behavior in the sticking coefficient.

We have completed a series of measurements of the heat capacity and sticking coefficient of ${ }^{4} \mathrm{He}$ on $\mathrm{Ag}$ films. The heat capacity measurements can be accurately fit by calculations assuming only a two-dimensional non-interacting bose gas. This is the first observation of bose statistics in the thermodynamics of adsorbed monolayers. In contrast to similar measurements on graphite substrates, there is no evidence for the formation of solid phases at the coverages and temperatures studied. The measured sticking coefficient 
is independent of temperature and coverage at low coverages, but abruptly drops at coverages of about 0.5 monolayers. The sticking coefficient data cannot be explained in terms of solidification or layer formation, due to the absence of corresponding anomalies in the temperature dependence of the heat capacity.

\section{$\mathrm{H}_{2}$ adsorbed on $\mathrm{Ag}$}

\section{Measurements}

Some preliminary measurements of the heat capacity of $\mathrm{H}_{2}$ adsorbed on $\mathrm{Ag}$ films were carried out. These measurements will be repeated and extended to lower temperatures and higher coverages between the completion of this thesis and my departure from Berkeley. These data were collected manually, and were distorted by electrical nonlinearities in the NTD-2 thermometers that were used in the calorimeter. A check of the $R(T)$ calibrations for these thermometers indicates that, because of the electrical nonlinearities, the recorded temperatures are $\sim 0.3 \mathrm{~K}$ too high. All of the data reported elsewhere in this thesis were obtained with more heavily doped thermometers, and at biases low enough that the electrical nonlinearities can be neglected. These calorimeters were assembled with less thermal conductance than the later calorimeters and were operated at frequencies below $50 \mathrm{~Hz}$, where the internal time constants are not as important. The temperature dependence of the hear capacity of the bare calorimeter exhibited $10 \%$ deviations from $\mathrm{AT}+\mathrm{BT}^{3}$ at the lowest temperatures investigated and fit well elsewhere.

A plot of the temperature dependence of the heat capacity for a series of exposures or $\mathrm{H}_{2}$ adsorbed on $\mathrm{Ag}$ is shown in Fig. 19. Al the highest temperature, desorption is responsible for the decreases in heat capacity. The upward deviations at the lowest temperature may be due to the beginning of solidification. 


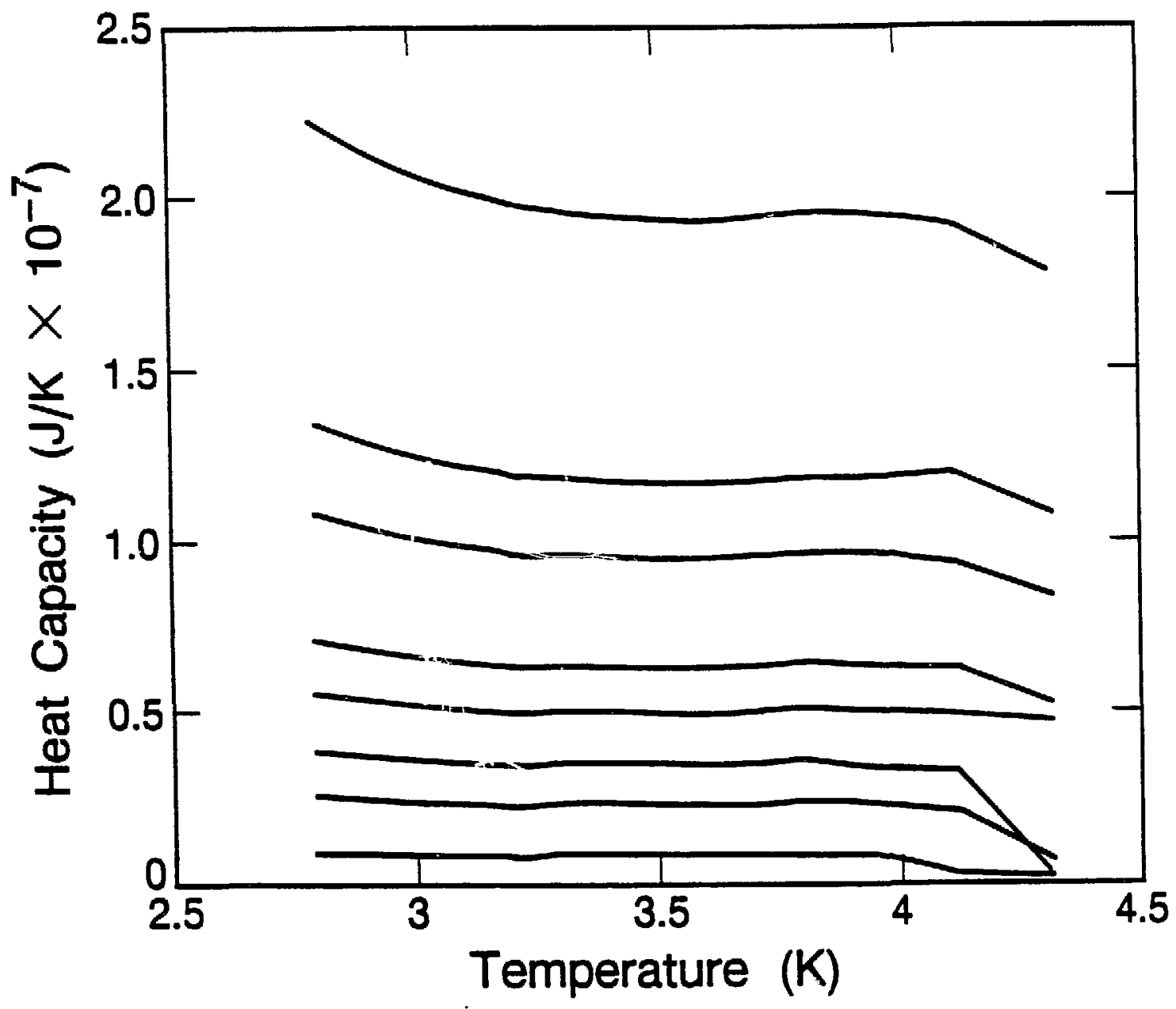

XBL 893.5066

Figure 19 Measurements of the temperature dependence of the heat capacity for a series of coverages of $\mathrm{H}_{2}$ on $\mathrm{Ag}$ films. Nonlinearities in the thermometers distorted the temperarure calibration for this calorimeter, leading to errors on the temperature axis of $10 \%$. 
The magnitude of the measured heat capacity due to the adsorbed $\mathrm{H}_{2}$ is very large. In the range of temperatures investigated, the adsorbed $\mathrm{H}_{2}$ has approximately as much heat capacity as the calorimeter!

A number of interpretations of these data are possible. The results may indicate the presence of a multilayer fluid, but $\mathrm{H}_{2}$ solidifies on all other substrates at temperatures in excess of $10 \mathrm{~K}$. Persistence of the fluid state to below $2.5 \mathrm{~K}$ would be a very surprising result. In has been suggested that $\mathrm{H}_{2}$ could form a superfluid if it could be prevented from solidifying at temperatures below $6 \mathrm{~K} .15$ Efforts to supercooi fluid $\mathrm{H}_{2}$ to much below $10 \mathrm{~K}$ have failed so far. ${ }^{16}$

Another interpretation of these results is that the electrical nonlinearities and internal time constants have distorted the measurement Such distortions wouid be multiplicative in nature. The observation of large adsorbate heat capacities would still hold true, indicating that something interesting is going on, whatever it might be. A series of reliable measurements is certainly necessary and will be carried out. 
References

1 E.A. Lynton, Superconductivity, Methuen \& Co. LTD, London p. 69 (1969).

2 T.E. Faber, Proc. Roy. Soc. (London) A241, 531 (1957).

3 A.E. Lange, S.H. Moseley, Private communication

4 Epo-Tek H20E, Epoxy Technology Inc., Billerica, MA.

5 T.W. Kenny and P.L. Richards, Bull. Am. Phys. Soc. 33, 264 (1988).

6 J. Ungaris et al, Surf. Sci. 109, 522 (1981).

7 J.K. Gimzewski et al, Phys. Rev. Lett. 55, 951 (1985).

8 E. Cheng, G. Ihm, and M.W. Cole, J. Low Temp. Phys. 74, 519 (1989).

9 O. Sinanoglu and K.S. Pitzer, J. Chem. Phys. 32, 1279 (1960).

10 A.D. McLachlan, Mol. Phys. 7, 381 (1964).

11 L.W. Bruch, Surf. Sci. 125, 194 (1983).

12 E. Zaremba and W. Kohn, Phys. Rev. B 15, 1769 (1977).

13 M.W. Cole, D.R. Frankl, and D.L. Goodstein, Rev. Mod. Phys 53, 199 (1981).

14 J.P. McKelvey and E.F. Pulver, Am. J. Phys. 32, 749 (1964).

15 V.L. Ginzburg and A.A. Sobyanin, Sov. Phys. JETP Lett. 15, 242 (1972).

16 H.J. Maris, G.M. Seidel, and T.E. Huber, J. Low Temp. Phys. 51, 471 (1983). 


\section{Euture measurements}

This apparans is capable of a number of measurements that are not possible elsewhere. In its current form an investigation of the properties of ${ }^{4} \mathrm{He}$ submonolayers on various substrates is possible. Gold films can be prepared by evaporation and annealing to $50 \mathrm{C}$ that are highly uniform. The He-Au attraction is approximately $40 \%$ stronger than for $\mathrm{Ag}$, but still substantially weaker than on graphite. It would be interesting to see if the properties of $\mathrm{He}$ on $\mathrm{Au}$ are similar to $\mathrm{He}$ on graphite, $\mathrm{Ag}$ or somewhere in between. Other metals could also be investigated, such as $\mathrm{Cu}, \mathrm{Pt}, \mathrm{Pd}, \mathrm{In}, . .$. The surface structures for these materials are less certain, but STM measurements could also be performed. The importance of the uniformity of the substrate could be investigated by studying ${ }^{4} \mathrm{He}$ submonolayers on rough (cold-deposited) Ag films.

All of these investigations could also be carried out for $\mathrm{H}_{2}$ monolayers, pending the results of the $\mathrm{H}_{2}$ on $\mathrm{Ag}$ experiments still to be completed.

\section{Possibilities at lower temperatures}

The technique is currently constrained to temperatures above $1.7 \mathrm{~K}$ by the ${ }^{4} \mathrm{He}$ cooling. Extension to lower temperatures through the use of ${ }^{3} \mathrm{He}$, dilution refrigeration, or adiabatic demagnetization would allow another series of experiments to be carried out. Monolayers of ${ }^{4} \mathrm{He}$ are expected to exist in a solid phase at $0 \mathrm{~K} .{ }^{1}$ At temperatures above $1.7 \mathrm{~K}$, we have found them to occupy a gas phase, which can be modeled very accurately with bose statistics. Somewhere between 1.7 and $0 \mathrm{~K}$, the solidification should occur, and an investigation of that phase transition would be very interesting.

Monolayers of ${ }^{3} \mathrm{He}$ on graphite are described by fermi statistics, but the case on $\mathrm{Ag}$ substrates may be qualitatively different, as for ${ }^{4} \mathrm{He}$ on graphite. Magnetic ordering 
transitions also appear in ${ }^{3} \mathrm{He}$ monolayers at temperatures near $100 \mathrm{mK}$, and could be studied on non-magnetic substrates (such as $\mathrm{Ag}$ ) as well as magnetic substrates (such as $\mathrm{Fe}$ or Ni). For these systems, the freedom to vary the character of the substrate could be extremely beneficial. 


\section{References}

1 E.Cheng, G. Ihm, and M.W. Cole, J. Low Temp. Phys. 74, 519 (1989). 


\section{Conclusion}

We have designed and built an apparatus capable of measuring the heat capacity of submonolayers adsorbed on evaporated films. The apparatus has been used in a number of investigations, and is particularly suited to measure.nents of ${ }^{4} \mathrm{He}$ submonolayers adsorbed on metallic films. Measurements have shown that, in contrast to ${ }^{4} \mathrm{He}$ adsorbed on graphite, the ${ }^{4} \mathrm{He}$ monolayers on $\mathrm{Ag}$ occupy a two-dimensional ideal gas phase at high temperatures. At lower temperatures, the first correction to the ideal classical gas that appears is due to Bose statistics. No other adsorbate-substrate combination has been shown to exhibit Bose statistics. This result clearly demonstrates the importance of the substrate in studies of this kind and illustrates the need for further studies on other substrates. Our approach to measurements of this kind allows an investigation of the effects of different substrates, and should be extended to lower temperatures. 
Appendix I Bias induced nonlinearities in neutron transmutation doped Ge at 4 He temperatures.

\begin{abstract}
During early attempts at ac calorimetry with Neutron Transmutation Doped (NTD) Ge thermometers, it was assumed that the resistance of any given thermometer was a function of the temperature alone, and that the electrical response was fast enough to follow the applied thermal oscillation. These assumptions were correct for the most heavily doped NTD thermometers, and for the so-called "Tinkham material" which was used for the first calorimeters. These assumptions are incorrect, however, for the lighter dopings typical of NTD-1 through 3. Carrier transport occurs in these materials by hopping conductivity, which gives rise to a dependence of the resistance upon the applied electric field as well as upon the temperature. An internal time constants has been observed which is not understood, but aiso may be related to hopping conductivity because of its occurrence in other hopping systems.
\end{abstract}

\title{
Introduction
}

Doped single-crystal germanium is widely used in the fabrication of resistance thermometers, which operate near ${ }^{4} \mathrm{He}$ temperatures. 1,2 Under proper conditions, the resistivity of these materials varies rapidly with temperature, and there is little or no excess noise. Small thermometers can be made with relatively low heat capacity and high internal thermal conductance. These properties alone qualify doped germanium as excellent thermometer material. Highly uniform dopant concentrations can be achieved through Neutron Transmutation Doping (NTD), in which Ge atoms are transmuted by nuclear reactions with thermal neutrons. 3 By changing the doping density, the resistivity at a given temperature can be controlled, allowing the fabrication of sensitive thermometers 
with impedances that are compatible with low-noise amplifiers. Such thermometers have been successfully used as the thermal sensing elements of bolometric infrared detectors ${ }^{4}$ as well as differential mictocalorimeters. 5

Typical bolometric radiation detectors consist of an energy absorbing element and a current-biased electrical resistance thermometer. These components are connected to a heat sink by a known thermal conductance. The thermal response time of the device is determined by its heat capacity and the thermal conductance. Characterization of such bolometers often begins with the measurement of a dc I-V curve. ${ }^{6}$ For a given thermal conductance to the heat sink, there exist bias currents small enough that the temperature rise due to dissipated electrical power can be neglected. For these small currents, the thermometer resistance should be independent of the bias current and the I-V curve is said to be ohmic. As the bias power is increased, a temperature difference appears between the thermometer and the heat sink, giving rise to a change in its resistance, and a non-ohmic, thermal nonlinearity in the I-V curve. In practical applications, a small amount of signal power is dissipated in the detector, leading to an additional temperature rise, and a correspondingly small change in the voltage across the thermometer. The change in voltage is linearly related to the applied signal power.

The absolute sensitivity of the bolometer can be determined from the shape of the I-V curve 6.7 if the electrical power dissipated in the thermometer produces the same result as signal power dissipated in the absorbing element, and if all of the nonlinearity in the I-V curve is of thermal origin. However, the changes in resistance observed in an I-V curve cannot always be attributed to temperature changes. Conduction in the thermometers under study is expected to be due in part to variable range hopping. ${ }^{3}$ For this conduction mechanism, electric fieids can induce nonlinearities, 8,9 and many theoretical discussions of these effects have been published. ${ }^{10-13}$ 
It usually is advantageous to modulate the incoming signal in order to reduce the importance of low frequency noise. The response of the detector as a function of modulation frequency must be known in order to obtain quantitative results. The frequency dependence of the response usually is ascribed to the thermal time constant of the detector or, occasionally to the electrical time constant of the measurement circuit. We have found that some thermometers have an effective intemal relaxation time that is longer than either the thermal time constant or the electrical time constant. In order to isolate internal relaxation times from external thermal or electric r relaxation times, a number of different thermometers were measured in the same thermal and electrical circuits.

In this work, the resistance of several NTD germanium resistance thermometers has been measured as a function of temperature, bias voltage, thermal oscillation frequency and dopant concentration. The results have been compared to the theoretical predictions found in the literature and to existing experimental data. The importance of these effects to absolute measurements of temperature, infrared radiation, exotic particles and heat capacity is discussed.

\section{Experimental Technique}

The NTD thermometers used in this experiment were fabricated from ultrapure Ge single crystals, which were doped to the acceptor and compensating donor concentrations as shown in Table 1 by exposure to a thermal neutron source. ${ }^{3}$ This procedure takes advantage of the uniformity of the neutron source and the small transmutation crosssections to produce very uniform dopant concentrations. In contrast, both implantation doping and melt doping of semiconductors can produce uneven dopant concentrations, which greatly complicates the problem of obtaining a suitable thermometer. The doped $\mathrm{Ge}$ crystals are sliced into $300 \mu \mathrm{m}$ wafers, fine-lapped, and etched in a $4: 1 \mathrm{HNO}_{3}: \mathrm{HF}$ solution 
to remove lapping damage, provide clean surfaces for ion implantation and to insure contact adhesion. Both surfaces are siep-implanted with $B^{+}$ions $\left(1 \times 10^{14} \mathrm{~cm}^{-2}\right.$ at $25 \mathrm{keV}$, $2 \times 10^{14} \mathrm{~cm}^{-2}$ at $50 \mathrm{keV}$ ), which produces a metallic region in the Ge approximately $2000 \AA$ deep. A $200 \AA$ layer of Pd and a $4000 \AA$ layer of $A u$ are argon sputtered onto the wafers, which are then annealed for one hour at $300 \mathrm{C}$. Annealing helps activate the implanted ions, and relieves any stress resulting from the metallization. The wafers were cut into chips $0.265 \mathrm{~mm}$ on a side, and rinsed briefly in the $4: 1$ solution to etch the damaged surfaces and thus remove surface electronic states, which might irreproducibly contribute to conduction at low temperatures. The final size after all processing is approximately ( 0.25 $\mathrm{mm})^{3}$.

Several different NTD thermometers were mounted in each test apparatus as is shown in Fig. 20. The thermometers were attached to evaporated $\mathrm{NiCr}$ films on a sapphire substrate with an Ag-filled conductive epoxy. 14 Copper wires with a diameter of $25 \mu \mathrm{m}$ were also attached to the thermometers and to the films with conductive epoxy. The other ends of the wires were soldered to Cu heat sink posts. This assembly, which was designed for other purposes, 5 is a useful test bed for the comparison of nonlinearities of two thermometers because of the ability to do simultaneous tests in nearly identical thermal surroundings.

\section{Measurements}

A 9 volt mercury battery and a ten-turn $10 \mathrm{k} \Omega$ potentiometer produced a variable dc bias voltage across a room temperature carbon load resistor in series with a cold thermometer. Both the total bias voltage and the voltage across the thermometer were measured with digital voltmeters with input impedance $>1 G \Omega$ and recorded manually. Because of the large thermometer impedances studied, two-wire measurements of resistance were sufficiently accurate, and the lead resistances have been neglected. To 


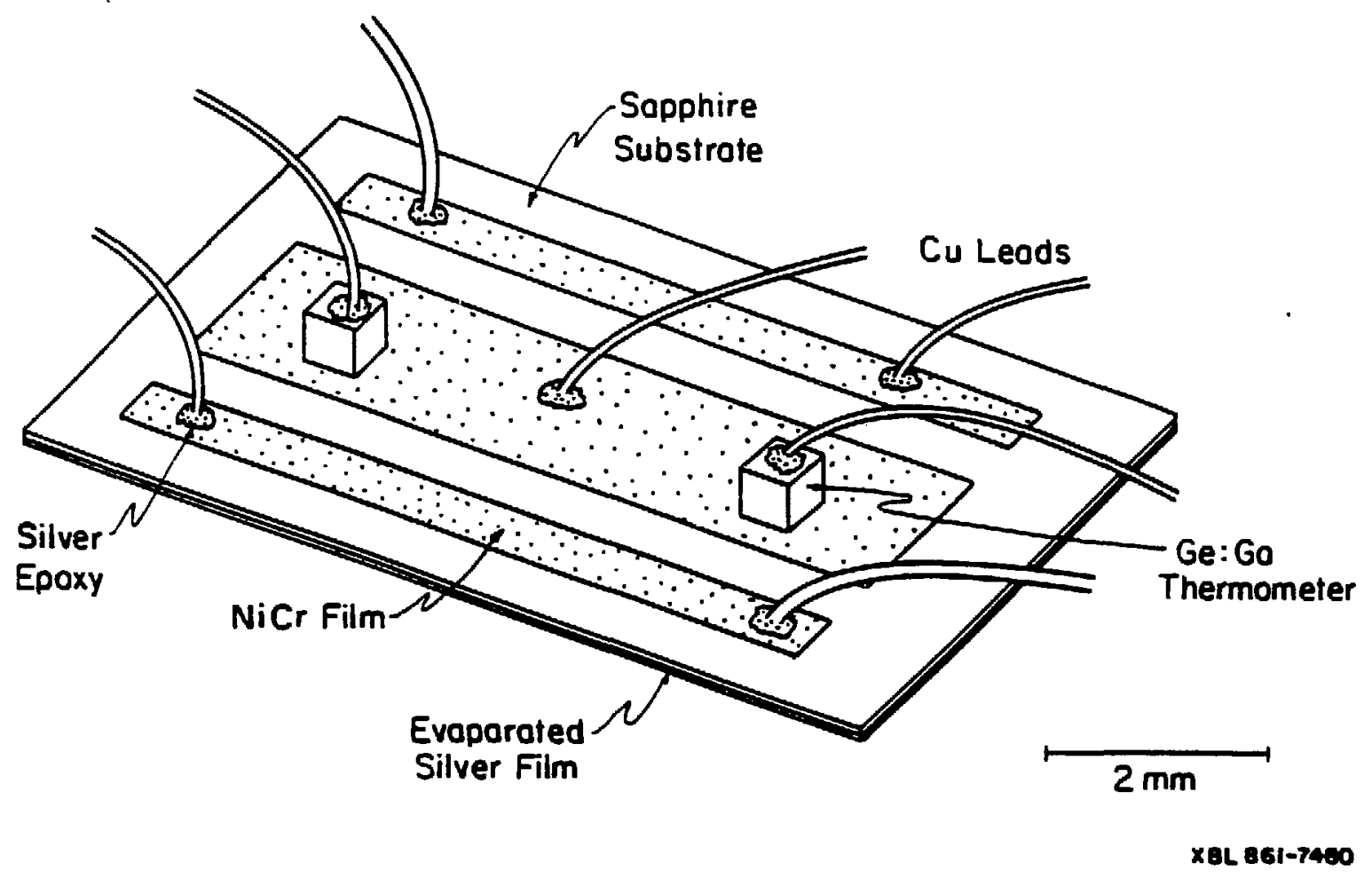

Figure 20 Schematic diagram of the test apparatus used in the measurements. All contacts to the wires, thermometers and $\mathrm{NiCr}$ films were made with Ag-filled conductive epoxy. As many as four thermometers can be mounted on a single assembly and tested simultaneously. 
check for thermal EMFs, the polarity of the bias was periodically reversed. Nonlinearities from non-ideal contacts are possible. Measurements with separate current and potential contacts were not made. The symmetry of the data with respect to bias direction showed that contact variability was not a problem.

Measurements of the dependence of the resistance on thermoratter lattice temperature and de bias voltage were carried out with the test apparatus submerged in liquid ${ }^{4} \mathrm{He}$. The thermal conductance to the heat sink is then limited by the thermal boundary resistance between the Ge surfaces of the thermometers and the liquid $+\mathrm{He}$, and is of order $10^{-3} \mathrm{~W} / \mathrm{K}^{15}$ The vapor pressure of the helium bath was measured with a capacitance manometer and was used to calculate the bath temperature. The temperature of the helium bath was controlled by observing the vapor pressure and manually adjusting the valve to the vacuum pump. Drifts in the bath temperature were less than $5 \%$ during these measurements. The errors introduced by these drifts do not affect the major conclusions of this study.

As the applied electric field approaches zero, the resistivity of these materials approaches a constant vaiue, which depends on the temperature. For variable range hopping in a disordered semiconductor, one expects to find low-field resistivities which vary as 16

$$
R(0, T)=R_{0} \exp \left(T_{0} / T\right) \alpha,
$$

where $\alpha$ is equal to 0.25 if one assumes that the medium is three-dimensional and that the carrier density of states near the Fermi surface is constant. However, it has been shown that long range Coulomb interactions between localized electrons can give rise to an energy gap between the filled and empty states. 17,18 The resulting quadratic variation in the 
density of states near the Fermi surface leads to a low-temperature resisivity as in Eq. 21, but with $\alpha=0.5$. At higher temperatures, where $\mathrm{k}_{\mathrm{B}} \mathrm{T}$ is comparable to the Coulomb gap, deviations from this temperature dependence are expected. Temperature dependent resistivities as in Eq. 21 with $\alpha=0.5$ have been observed experimentally, 19,20 and a review of all these considerations for the case of doped Ge has been given by Efros and Shklovskii. 21 The low-field resistivity of the four samples studied is shown in Fig. 21a as a function of $T^{-1 / 2}$. At the lowest temperatures studied, the resistivities of all four samples as plotted in Fig. 21a approach straight lines. This is consistent with previous low temperature measurements on similar samples ${ }^{3}$, and with the predictions for variable range hopping in the presence of a Coulomb gap. As the temperature increases, the deviations from straight lines indicate the presence of a change in the conduction mechanism. At the highest temperatures, the data are better fit by Eq. 21 with $\alpha=1$, as shown in Fig. 21 b. This suggests that conduction due to ionization of bound carriers is becoming important.

As the electric field is increased, deviations from ohmic behavior become apparent. A series of I-V curves for a typical thermometer measured at different temperatures in the liquid helium bath are shown in Fig. 22. Since the resistance of these thermometers depends on temperature, these curves might be interpreted as evidence for increases in thermometer temperature due to dissipated bias power. However, quantitative measurements do not support this hypothesis. For example, in the I-V curve measured at 1.25 $\mathrm{K}$ for sample NTD-2, a nonlinearity of a few percent is observed at an applied bias power of less than $10^{-9} \mathrm{~W}$. Since the thermal conductance between the thermometers and the bath is of order $10^{-3} \mathrm{~W} / \mathrm{K}$, the expected temperature rise is $10^{-6} \mathrm{~K}$. This should produce a change in resistance of about 1 part in $10^{6}$, which is only $10^{-4}$ of the observed effect. This leaves only the possibility that the resistance depends on the applied bias voltage as well as the temperarure. 

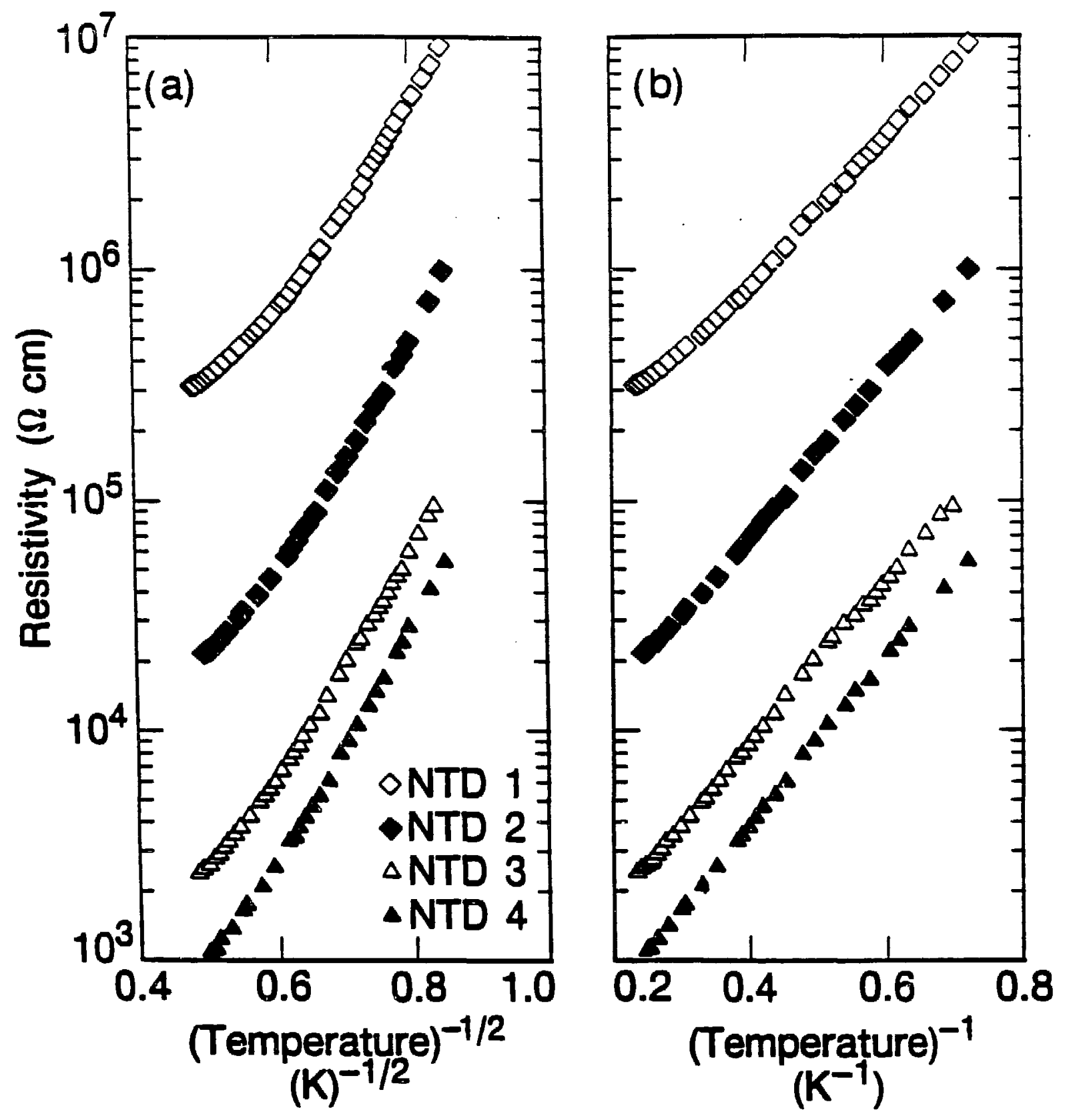

XEL

Figure 21 Plots of the log of the resistivity of all four thermometers measured as a function of (Temperature) ${ }^{-\alpha}$ with $\alpha=1 / 2$ in (a) and $\alpha=1$ in (b). 


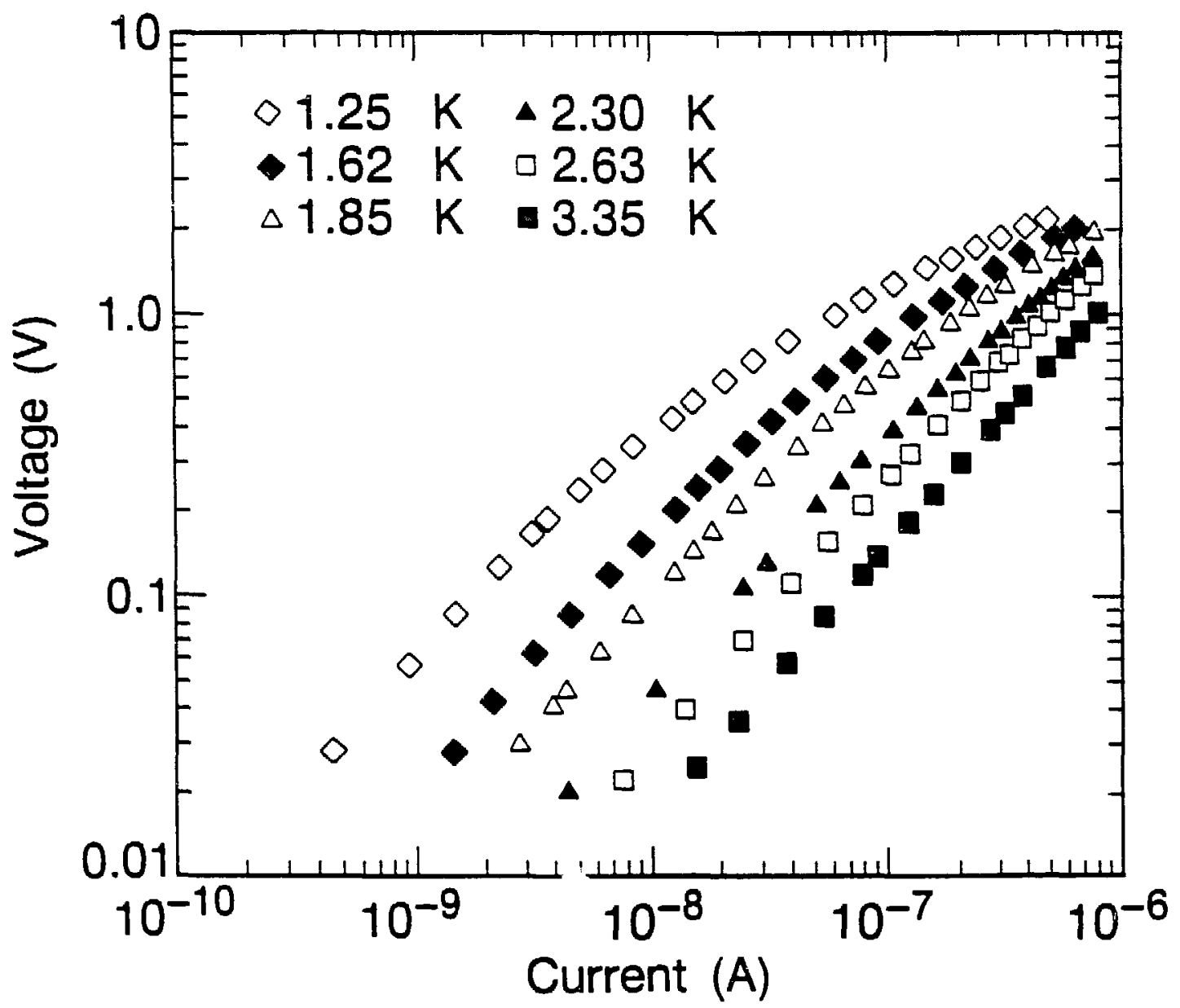

XBL 884-73691

Figure 22 A series of I-V curves for thermometer NTD-2 submerged in ${ }^{4} \mathrm{He}$ at 6 different bath temperanures. Nonlinearities are evident for applied power as low as $10^{-9} \mathrm{~W}$. Heating of the thermometer by the bias power is significant only above $10^{-5} \mathrm{~W}$. 
Much thecietical work has gone into the understanding of electrical nonlinearities in hopping conduction, and the predictions ${ }^{10-13}$ are that the resistance depends on the electric field

$$
R(E, T)=R(0, T) \exp \left(-\mathrm{eEL} / k_{B} T\right)
$$

where $\mathrm{E}$ is the electric field, $\mathrm{k}_{\mathrm{B}}$ is Boltzmann's constant, and $\mathrm{L}$ is a characteristic length for the hopping process. Different authors predict additional numerical factors in the exponent, but all find a form similar to that in Eq. 22. To check for such a field-dependent nonlinearity, the I-V curves in Fig. 22 were re-plotted as shown in Fig. 23. At each bath temperature, the data fit straight lines, indicating that the measured nonlinearities are consistent with Eq. 22. The non-zero slopes indicate that there is an electric field-induced nonlinearity in all of the samples throughout the range of temperatures and electric fields investigated. The slight deviations from straight lines in Fig. 23 are within the drifts expected rrom bath temperature variations during a particular measurement, and do not recur.

As the bath temperature is changed, the slope of the lines through the data in Fig. 23 also changes, implying that the characteristic hopping length $L$ has a temperature dependence. Fig. 24 shows a plot of the temperature dependence of $L$ for thermometers with three different doping densities. The nonlinearity in Eq. 22 is most important when the characteristic hopping length is large and the temperature is low. Therefore, measurements below $1 \mathrm{~K}$ will be most seriously affecte $\perp$ Surprisingly, the samples with the largest average impurity separation show the smallest characteristic hopping lengths. The characteristic hopping length in variable range hopping in the presence of a Coulomb gap is expected 21 to vary as $\mathrm{T}^{-1 / 2}$ at low temperatures. This prediction is also plotred in Fig. 24 and is not in good agreement with the data. 


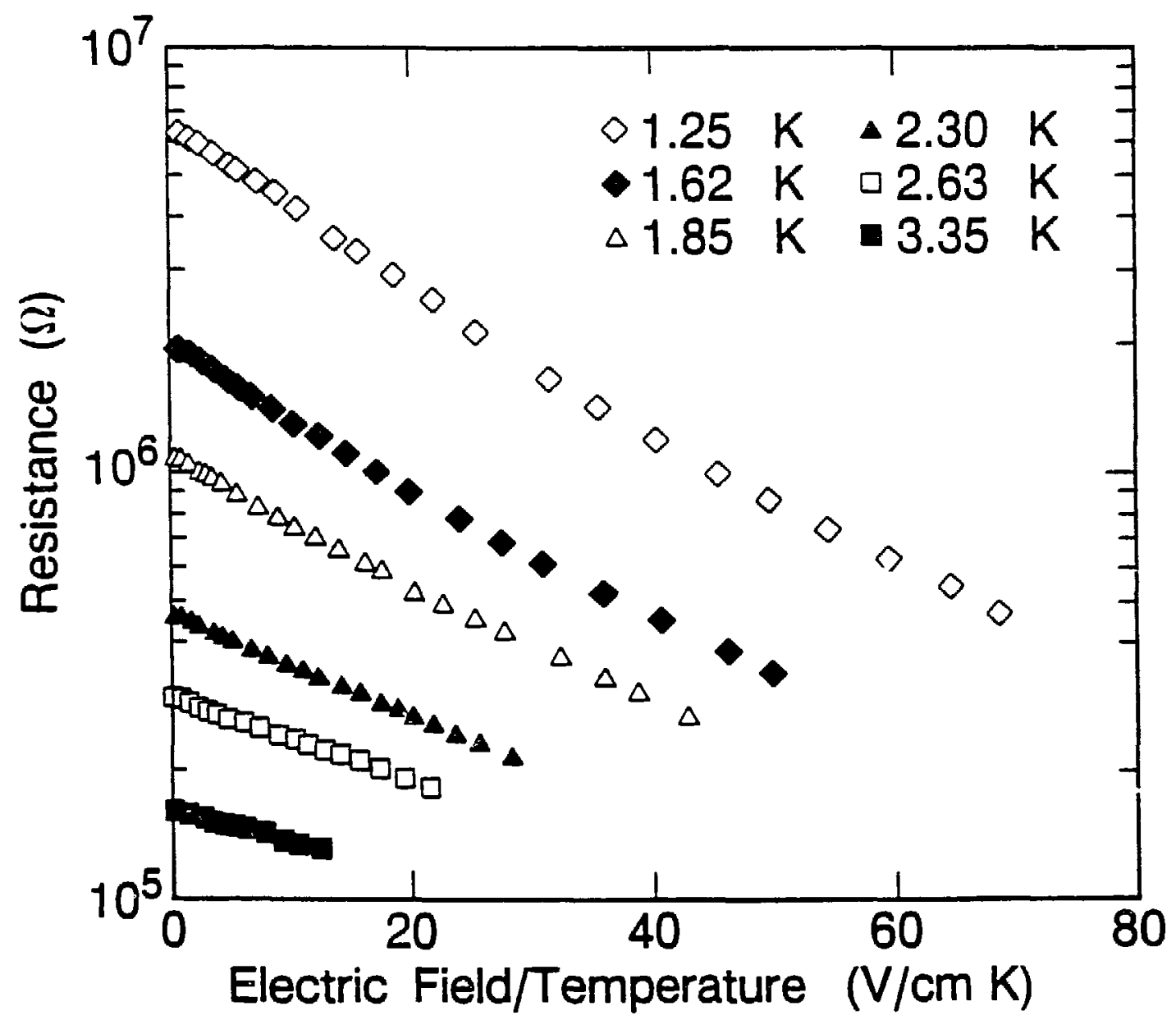

XEL 884-7367A

Figure 23 Data from Fig. 22 re-plotred as the log of the resistance versus the ratio of electric field to temperature. Straight lines are consistent with theorerical predictions for electric field assisted hopping. 


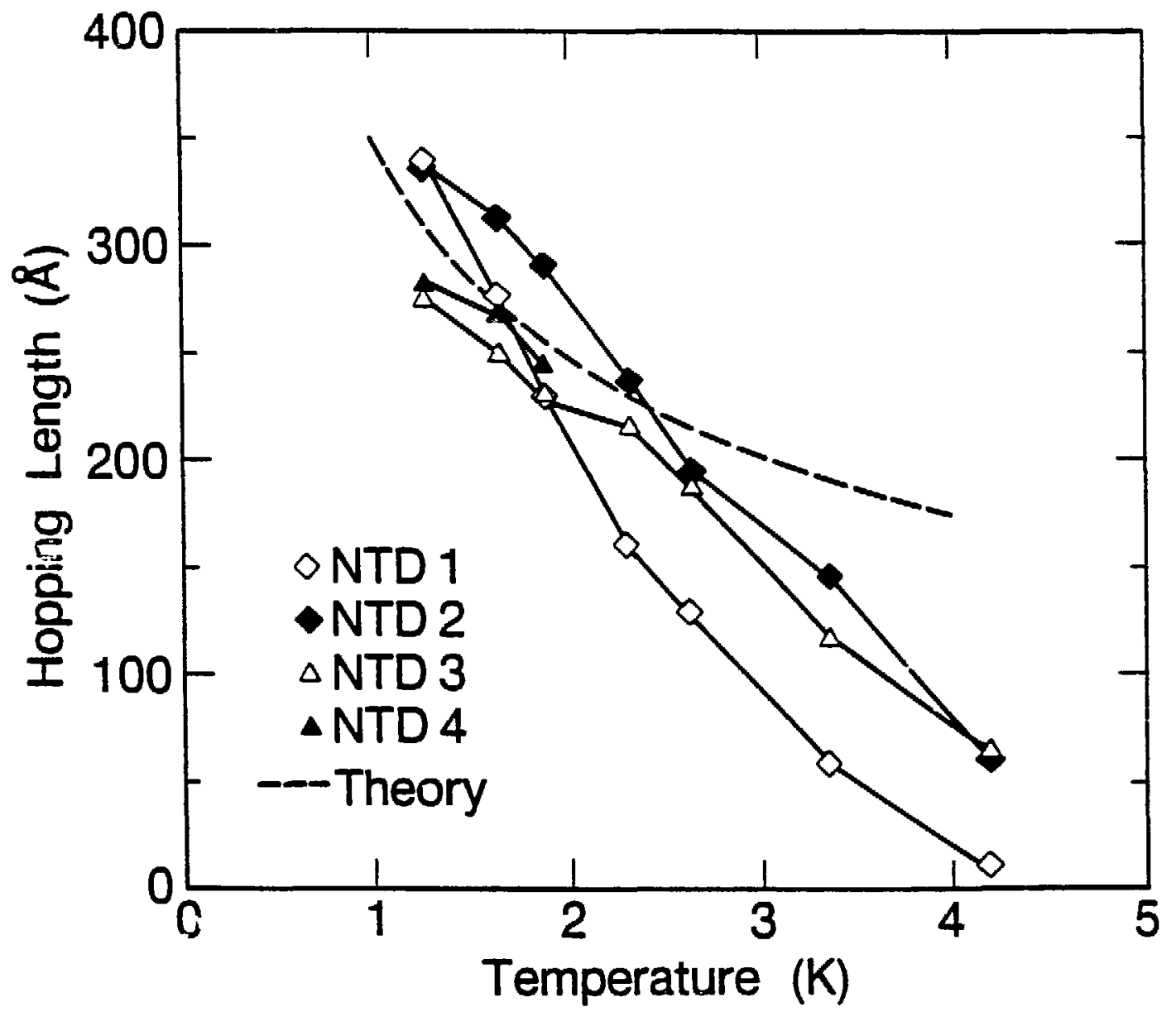

XEL-884 737

Figure 24 Temperature dependence of the characteristic hopping length $L$ extracted from I-V curves for ali of the thermometers in this srudy. The lines drawn through the data are included as a guide to the eye. The dashed line corresponds to the predicted $\mathrm{T}^{-1 / 2}$ deperidence of the characteristic hopping length upon temperature, and has been fitted to the low temperature data. 


\section{AC Measurements}

The phase and amplitude of the voltage resulting from an ac thermometer current bias were measured as a function of frequency for several different thermometers with different resistances with the samples submerged in superfluid ${ }^{4} \mathrm{He}$. The results were consistent with an RC rolloff due to a capacitance of approximately $300 \mathrm{pF}$.

When the test apparatus is placed in a vacuum and an oscillaring current is applied to the heating strip, there is an oscillation in the resistance of the thermometer. If the thermometer response is sufficiently fast, the amplitude of the resistance oscillation is determined by the modulation frequency, heat capacity of the test apparatus, and the thermal conductance to the heat sink 22 Two thermometers mounted on the same test apparatus would then have the same thermal response. Fig. 25 shows the frequency dependence of the amplitude of the voltage oscillation of four current-biased thermometers mounted on the same substrate. The data for NTD-4 are well represented by a simple rolloff $\left(1+\omega^{2} \tau^{2}\right)^{-1 / 2}$ with a time constant $\tau_{1}=C / G=1.3 \mathrm{~ms}$ up to $2 \mathrm{kHz}$. This value of the thermal time constant is consistent with the known values of $C$ and $G$ for the test apparatus. Above $2 \mathrm{kHz}$, the rolloff steepens because of the electrical time constant of 0.1 ms. Additional measurements on very heavily melt-doped thermometers are coincident with those for NTD-4, the most heavily doped thermometer in this study. Since the curve for NTD-4 shows the fastest response and is coincident with that of a completely different thermometer, it reflects the thermal response of the test apparatus. The data for NTD-1 and 2 are well represented at all temperatures by the function $\left(1+\omega^{2} \tau_{1}^{2}\right)^{-1 / 2}\left(1+\omega^{2} \tau 2^{2}\right)^{-1 / 2}$ with $\tau_{1}=1.3 \mathrm{~ms}$ and $\tau_{2} \approx 4 \mathrm{~ms}$. The second time constant is attributed to an internal relaxation in the thermometer. The data for NTD-3 is more complicated but also shows the effects of the internal relaxation. Measurements on a given themometer at different temperatures. and therefore different resistances, but the same capacitance, produce 


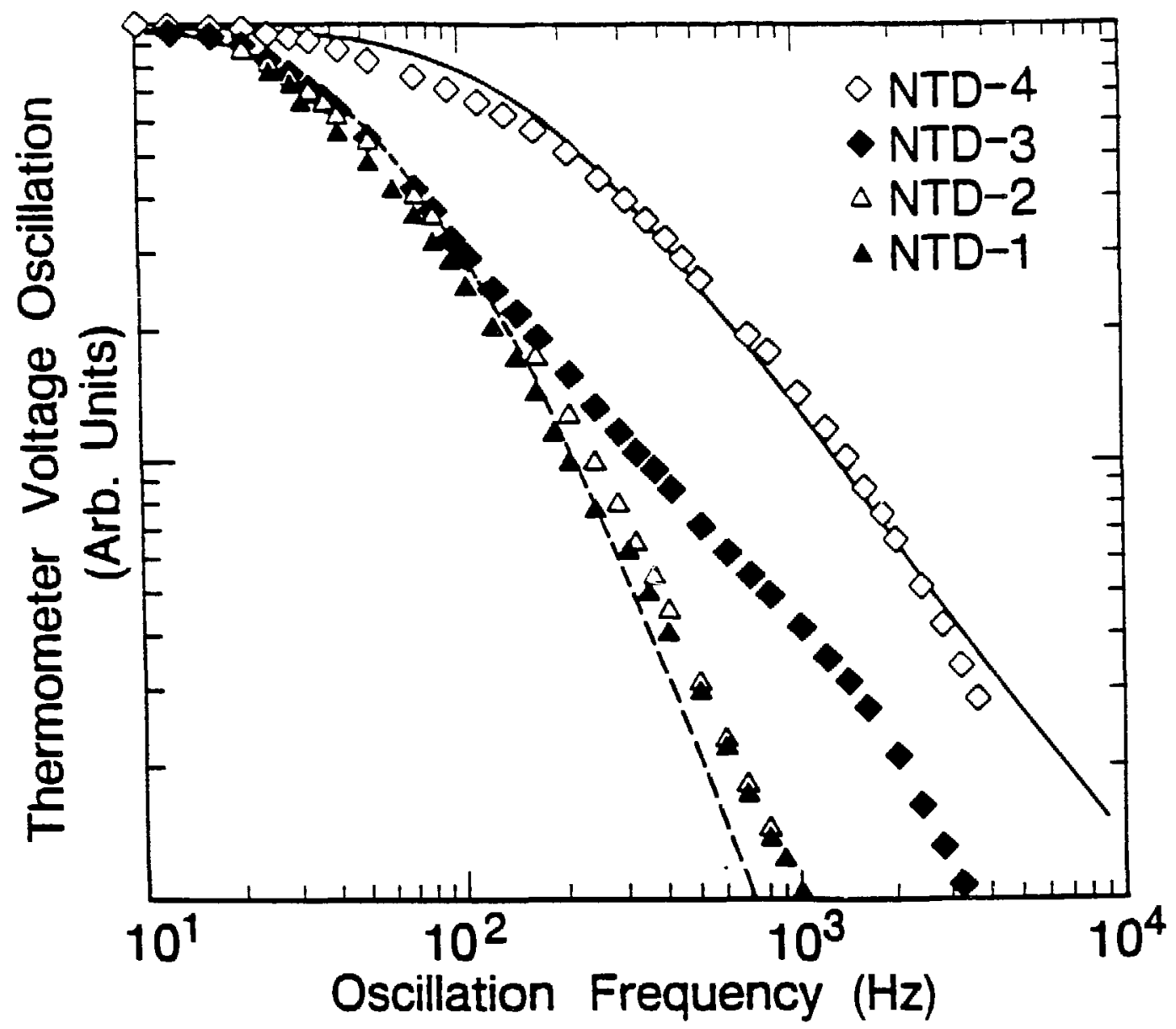

XBL B84-7368

Figure 25 The measured amplitude of the voitage oscillation of the thermometer plotted as a function of the applied thermal oscillation frequency for four thermometers mounted on the same substrate. The solid line is the expected rolloff for a $1.3 \mathrm{~ms}$ time constant, while the dashed line is the product of the rolloff from a $1.3 \mathrm{~ms}$ time constant and the rolloff from a $4 \mathrm{~ms}$ time constant. The thermal time constant of the apparatus is $\tau_{1}=1.3 \mathrm{~ms}$. An intemal relaxarion effect in NTD- 1 and 2 can be described by an additional time constant $\tau_{2}$ $=4 \mathrm{~ms}$. Electrical RC times become important in all curves after the first decade of rolloff. 
virtually the same frequency dependences. This is further indication that the internal time constant is not due to the rolloff of the electrical circuit.

Electrothermal feedback can occur in measurements of this type, giving rise to apparent changes in the thermal conductance between the test apparatus and the heat sink. For the small thermometer biases used in these measurements, electrothermal feedback modifies the measured thermal conductance by less than $0.1 \%$, and its contribution has been ignored.

\section{Discussion}

At low applied electric fields, the low-temperature resistivity for conduction by variable range hopping in the presence of a Coulomb gap is expected to follow Eq. 21 with $\alpha=0.5$. The measured resistivities follow this dependence at the lowest temperatures investigated and at lower temperatures ${ }^{3}$ as predicted by Efros and Shklovskii. ${ }^{21}$ At higher temperatures, thermal ionization of carriers becornes important, as seen by the approach to an $\alpha=1$ dependence.

The electric field dependence of the resistance at any given temperature as shown in Figs. 22 through 24 is in agreement with the form predicted for variable range hopping. The rough consistency between the values of the hopping length and the average separation of uncompensated acceptors is encouraging, if not particularly illuminating. Agreement between the measured characteristic hopping length and average impurity separation has not always been found in other materials. ${ }^{9}$ The hopping length decreases more rapidly with temperature than is expected. This discrepancy may be taken as further evidence for a transition in the conduction mechanism from variable range hopping to another mechanism, such as thermally ionized carrier conduction. 
The appearance of an internal relaxation time greater than the thermal ime constants of these detectors is not expected. Simple arguments ${ }^{23}$ can be used to set a tight upper limit on the thermal relaxation time of the carriers in this material as follows. The heat capacity of the carriers must be less than that of an ideal gas of carriers whose number densiry equals that of the uncompensated acceptors in the material. If we interpret the observed nonlinearity in the I-V curves as a change in the electron temperature, we can estimate the thermal conductance from the electrons to the lattice. This procedure has been used elsewhere to estimate the thermal conductance between the electrons and phonons in doped Ge thermometers. ${ }^{2}$ From these models, the estimated heat capacity is $\mathrm{C}=10^{-12} \mathrm{~J} / \mathrm{K}$ and the estimated thermal conductance is $\mathrm{K}=10^{-8} \mathrm{~W} / \mathrm{K}$. The resulting time constant is $10^{-4} \mathrm{~s}$, which is considerably shorter than the observed internal relaxation time. Since the changes in resistance are more likely to be due to the field dependent nonlinearity, this represents an underestimate of the the true thermal conductance present in this system. Since the heat capacity is grossly overestimated, and the thermal conductance is probably underestimated, the true thermal relaxation time for the electrons should be much faster.

Because of phase space factors, the strength of the electron-phonon interaction is likely to be strongly temperature dependent, while the heat capacity of the electron gas is weakly temperature dependent. Therefore under this model, we would expect a strong dependence of the relaxation time with temperature, which is not observed. The mechanism for the internal time constant is not known.

McCammon and coworkers have observed discrepancies between expected and measured responses of $x$-ray detectors made from antimony doped silicon. 24 In their experiments, individual $M n K \alpha, x$-rays were absorbed, producing pulses in the temperature of the detector. The shapes of the measured pulses were not consistent with a single exponential thermal response, and may have been distorted by an internal time constant. 
They also report a field-induced nonlinearity whose form is consistent with our observations. The appearance of both the internal time constant and the field induced nonlinearity in these two different materials suggests that these effects may be fundamentally related. Similar measurements by Silver and Labov 25 on heavily doped NTD Ge at lower temperatures than in this study have been carried out. Their results indicate the presence of both a field induced nonlinearity, and an internal time constant similar to the effects reported here and by McCammon.

Loponen and coworkers ${ }^{26}$ have made extensive measurements of the timedependent heat capacity of amorphous semiconductors below $1 \mathrm{~K}$. Their measurements at ms time scales showed that the heat capacity had a component with a logarithmic time dependence. A time dependent heat capacity of this form is theoretically expected ${ }^{27}$ for glasses which exhibit tunneling excitations with a wide distribution of relaxation times and energies. A similar distribution in relaxation times could be responsible for the distortions in pulse shapes observed by others 24,25 , as well as the internal time constant reported here.

There have been many reports in the literature of frequency dependences in the AC conductivity of materials in the hopping conductivity regime. 28,29 One explanation of these results is that polarizability of the bound carriers increases with frequency and adds to the total ac conductivity of the material. A more complicated model, which includes the effects of spatial fluctuations on the ionization probability of deep levels appears to fit more of the observed details. 30 In the present work, measurements of the voltage response to an ac thermometer bias were consistent with an electrical time constant, and showed no clear evidence of any other frequency dependent contribution to the ac conductivity up to $10^{5} \mathrm{~Hz}$. 
The implications of these results for the use of NTD thermometers for absolute measurements of temperature, absorbed power, or heat capacity can be summarized as follows:

1) The electric field dependence of the resistance of these materials complicates their use as ordinary thermometers. In particular, great care must be taken to perform calibrations and measurements at identical voltages, or, preferably, in the low voltage limit, where the field dependence is not important. Another approach is through the use of electrical readouts that measure thermometer current at constant bias voltage. The electric field dependence of the resistivity is especially important at lower temperatures, where the hopping lengths can be quite large.

2) Calibrations of bolometric radiation detectors obtained by calculations based on measured I-V curves will be distorted by the electric field dependence. This is because the observed changes in resistance will not be entirely due to sample heating. Correction procedures for electrical nonlinearities have been proposed. 31 However, the observed temperature dependence of the hopping length shows that more complicated corrections may be required. Therefore, the only reliable calibrations of bolometric radianon detectors are those which deposit the energy in the same place in the thermal circuit as in the actual measurement.

3) The appearance of an intemal relaxation time larger than the thermal relaxation time of the apparatus and the RC time constant of the electrical circuit complicates any ac measurement made with these materials. In particular, the sensitivity of bolometric detectors to modulated or pulsed signals will be reduced by this effect. This implies that calibrations of absolute bolomerric detectors will have to be pertormed at the same frequency as the actual measurement. Furchermore, the sensitivity of bolometric detectors 
being designed for $\mathrm{x}$-ray detection $24,25,32$ and dark-matter searches ${ }^{33}, 34$ depends on fast response and so will be seriousiy reduced by the observed internal time constant. Finally, the application of these thermometers to ac calorimetry ${ }^{4}$ is seriously affected, since accurate measurements require that the slowest time constant be due to thermal relaxation of the entire calorimeter. The presence of an internal ime constant longer than the thermal time constant of the detector makes quantitative ac heat capacity measurements very difficult.

For a given bias power, the electric field-induced nonlinearity and the internal time constant decrease in more heavily doped material. This suggests that optimum performance can be obtained through the use of relatively low impedance thermometers. The detailed optimizations depend sensirively on the properties of amplifiers and are beyond the scope of this work.

\section{Conclusion}

A series of measurements have been made of the electrical properties of doped germanium thermometer material at ${ }^{4} \mathrm{He}$ temperatures. The temperature dependence of the low-field resistivity is in agreement with the predictions for variable range hopping in the presence of a Coulomb gap at the lowest temperatures studied. At higher temperatures, deviations from this prediction arise due to conduction by thermally ionized carriers. The electric field dependence of the resistivity has a form consistent with predictions for variable range hopping, but the magnitude and temperature dependence of the hopping length deduced are not correctly predicted. Finally, an internal relaxation time is observed which is longer than both the thermal time constant of the test appararus and the RC time constants in the electrical circuit. The observed intemal time constant can seriously distort the response of absolute thermal detectors. 
Table 1

\begin{tabular}{c|l|l|l|l} 
Dopant & NTD-1 & NTD-2 & NTD-3 & NTD-4 \\
\hline $\mathrm{Ga}$ & $2.21 \times 10^{15}$ & $4.41 \times 10^{15}$ & $6.62 \times 10^{15}$ & $9.94 \times 10^{15}$ \\
$\mathrm{As}$ & $6.28 \times 10^{14}$ & $1.26 \times 10^{15}$ & $1.89 \times 10^{15}$ & $2.83 \times 10^{15}$ \\
$\mathrm{Se}$ & $9.2 \times 10^{13}$ & $1.6 \times 10^{14}$ & $2.5 \times 10^{14}$ & $3.8 \times 10^{14}$ \\
$\mathrm{~N}_{\mathrm{a}}-\mathrm{N}_{\mathrm{d}}$ & $1.49 \times 10^{15}$ & $2.99 \times 10^{15}$ & $4.48 \times 10^{15}$ & $6.73 \times 10^{15}$
\end{tabular}

Table 1 Dopant concentrations in the NTD samples used in this study. All other impurity concentrations are below $10^{13}$. All concentrations in $\mathrm{cm}^{-3}$. 
References

1 N.S. Nishioka, P.L. Richards, D.P. Woody, Appl. Opi. 17, 1562 (1978).

2 E.P. Roth, J.R. Matey, A.C. Anderson, D.A. Johns, Rev. Sci. Instrum. 49, 813 (1978).

3 E.E. Haller, Infrared Phys. 25, 257 (1985).

4 A.E. Lange et al., Int. J. Infrared and Millimeter Waves 4, 689 (1983).

5 T.W. Kenny, P.L. Richards, Bull. Am. Phys. Soc. 33, 264 (1988).

6 R.C. Jones, J. Opt. Sci. Am. 43, 1 (1953).

7 J.C. Mather, Appl. Opt. 21, 1125 (1982).

8 J.M. Marshall, G.R. Miller, Philos. Mag. 27, 1151 (1973).

9 T.F. Rosenbaum, K. Andres, G.A. Thomas, Solid State Commun. 35, 663 (1980).

10 R.M. Hill, Philos. Mag. 19, 1307 (1971).

11 M. Pollak, I. Reiss, J. Phys. C 9, 2399 (1976).

12 N. Apsley, H.P. Hughes, Philos. Mag. 30, 963 (1974).

13 H. Bottger, V.V. Bryskin, Philos. Mag B 42,297 (1980).

14 Epo-Tek H20E, Epoxy Technology Inc., Billerica, Ma.

15 A.C. Rose-Innes, Low Temperature Laboratory Techniques (Elliot Bros. \& Yeoman Lid.,Speke, Liverpool, 1973), p. 106. This particular result is for $\mathrm{Cu}$ in ${ }^{4} \mathrm{He}$. Since this prediction is based on a phonon-velocity mismatch model and the phonon velocities for $\mathrm{Cu}$ and $\mathrm{Ge}$ are similar, the thermal boundary resistance for $\mathrm{Ge}$ in helium should also be similar.

16 N.F. Mott, Philos. Mag. 19, 835 (1969). 
17 M.L. Knotek, M. Pollak, Phys. Rev. B 9, 644 (1974).

18 A.L. Efros, B.I. Shkiovskii, J. Phys C 8 , L49 (1975).

19 A.G. Zabrodskii, K.N. Zinov'eva, Sov. Phys. JETP Lett. 37 (1983).

20 D. Redfield, Adv. Phys. 24, 463 (1975).

$2 \mathrm{i}$ A.L. Efros, B.I. Shklovskii, Electronic Properties of Doped Semiconductors (SpringerVerlag, New York, 1984), p. 202.

22 P.F. Sullivan, G. Seidel, Phys. Rev. 173, 679 (1968).

23 D. McCammon, private communication.

24 D. McCammon, S.H. Moseley, J.C. Mather, R.F. Mushotzky, J. Appl. Phys. 56, 1263 (1985).

25 E. Silver et al., "Recent Results in X-Ray Calorimetry at the Laboratory for Experimental Astrophysics", I.A.U.Colloquium No. 115 High Resolution X-Rav Spectroscopy of Cosmic Plasmas, Cambridge, August 22-25,1988.

26 M.T. Loponen, R.C. Dynes, V.Narayanamurti, J.P. Garno, Pkys. Rev. B 25, 1161 (1982).

27 P.W. Anderson, B.I. Haiperin, C.M. Varma, Philos. Mag. 25, 1 (1972).

28 M. Pollak, T.H. Geballe, Phys. Rev. 122, 1742 (1961).

29 H. Fritzsche, M. Cuevas, Phys. Rev. 119, 1230 (1960).

30 B. Pistoulet, F.M. Roche, S. Abdalla, Phys. Rev. B 30, 5987 (1984).

31 J.C. Mather Appl. Opt. 23, 3181 (1984).

32 S.H. Moseley, J.C. Mather, D. McCammon, J. Appl. Phys. 56, 1257 (1985).

33 B. Cabrera, L.M. Krauss, F. Wilczek, Phys. Rev. Lett. 52, 25 (1985).

34 B. Sadoulet, "Prospects for Detecting Dark Matter Particles by Elastic Scattering", in Proceedings of the 13th Texas Symposium on Relativistic Astrophysics, Chicago, Dec. 14-19, 1986, Ulmer, M. L., Editor (World Scientific, Singapore, 1987), p. 260. 
Anoendix $\amalg$ Generation of intense tunable nicosecond oulses in the farinfrared

\begin{abstract}
Continuously tunable far-infrared (FIR) picosecond pulses have been generated frow $20-200 \mathrm{~cm}^{-1}$ through the parametric interaction between two visible pulses. Pulse energies up to $3 \mathrm{~nJ}$ were detected. The quantum efficiency $0.1-0.3 \%$ was surprisingly constant over this frequency range considering the large variations in FIR absorption, diffraction, and phonon resonar, e enhancement. The depletion of the visible pulse and the depeidence of FIR energy on pump power were typical of a saturated process. However, the FIR energy showed unexpectedly large pulse-to-pulse fluctuations. Understanding of the origin of these fluctuations may lead to substantial improvement in the average efficiency.
\end{abstract}

\title{
Introduction
}

The investigation of dynamical processes in the optical has benefited greatly from the substanial recent progress in the development of pulsed dye lasers. Similar developments in the far-infrared (FIR) have not occurred, largely due to the difficulties in the construction of FIR lasers. As a result, studies of dynamical processes in the FIR have relied upon indirect measurements.

Techniques that apply the developments in the optical to the generation of FIR pulses have been widely investigated. Optical rectification has been used to generate broadband IR picosecond ${ }^{1}$ and femtosecond ${ }^{2}$ pulses, but with low intensity. Recently, there has been interest in the generation of picosecond FIR from free electron lasers, but success has not yet been demonstrated ${ }^{3}$ A variety of other techniques have been successful in generating longer FIR pulses. 4,5 In particular, tunable, nanosecond FIR 
pulses have been generated by difference frequency mixing of two visible dye lasers. 6,7 The recent development of high-power, picosecond dye lasers has allowed an extension of this technique to the picosecond domain. This letter reports the generation of highintensity, narrow-bandwidth, picosecond FIR pulses by this technique.

\section{Experimental}

In these experiments, two visible pulses, the first 1-2 ps long, and the second $5 \mathrm{~ns}$ long, were mixed in a $\mathrm{LiNbO}_{3}$ crystal to generate FIR pulses at a $10 \mathrm{~Hz}$ rate. Since mixing only occurred when the pulses overlap, picosecond FIR was generated. The $0.2 \mathrm{~mJ}$ picosecond pulses at $589 \mathrm{~nm}$ were generated by an amplified, synchronously-pumped dye laser. ${ }^{8}$ A commercial YAG-pumped dye laser produced the $20 \mathrm{~mJ}$ nanosecond pulses, which were tuned from $590-596 \mathrm{~nm}$. The pulse-to-pulse fluctuations were $10 \%$ for the picosecond pulses and 6\% for the nanosecond pulses. A single doubled YAG beam was split to pump both the nanosecond dye laser and the picosecond amplifier, thus insuring synchronization of the two pulses. The two beams were focused with $a>1 \mathrm{~m}$ focal length and overlapped at an angle of $5-50 \mathrm{mrad}$ in the $\mathrm{LiNbO}_{3}$ crystal. Spot sizes were $0.6 \mathrm{~mm}$ and $1.2 \mathrm{~mm}$ for the picosecond and nanosecond beams respectively. Smaller spot sizes caused crystal damage. Note that the instantaneous nanosecond intensity $\left(350 \mathrm{MW} / \mathrm{cm}^{2}\right)$ is much lower than the picosecond intensity $\left(70 \mathrm{GW} / \mathrm{cm}^{2}\right)$.

The arrangement of the experimental apparatus is shown in Fig. 26. Noncollinear phase matching was achieved by the method of Yang, et al. ${ }^{6}$ The two visible beams, as well as the FIR were polarized along the optic axis of the $\mathrm{LiNbO}_{3}$ to utilize the largest nonlinear coefficient. ${ }^{9}$ Since the FIR spectral band is below the optical phonon modes of the mixing crystal, the FIR refractive index $10(n=5.5)$ is much larger than the visible refrative index $(n=2.3)$. Therefore, the phase-matching can be achieved geometrically, without using birefringence. Tuning was achieved by changing both the angle between the 
visible beams and the difference in their frequencies. Because the FIR is produced at a 68 degree angle from the visible beams, a comer of the $4 \mathrm{~mm}$ cubic crystal was removed to allow the FIR to exit with small renlection losses. ${ }^{6}$ An advantage of the phase matching scheme used here over collinear phase marching is that the direction of the optic axis of the crystal does not have to be changed as the FIR is tuned. Thus, only one crystal is needed for the entire tuning range.

The FIR was produced with a significant divergence due to the focusing of the visible beams, diffraction of the FIR, and the bandwidth of the picosecond pulse. This divergence was increased by refraction at the crystal surface to $>1 \mathrm{srad}$. In order to collect a large solid angle, the small end of a Winston concentrator ${ }^{11}$ was placed in contact with the exit face of the crystal. The concentrator collected and collimated the emerging FIR pulse which then passed through band limiting filters to a composite infrared bolometer operated at $1.5 \mathrm{~K}$. A step-scanned Michelson Fourier spestrometer was used to measure the spectrum of the FIR pulses.

Figure 27a shows the median collected FIR energy at frequencies from $20-200 \mathrm{~cm}^{-}$ 1. The energies were typically $3 \mathrm{~nJ}$ per pulse. Figure $27 \mathrm{~b}$ shows the quantum efficiency for conversion of photons from the visible picosecond pulse to detected FIR photons. Surprisingly, there was no strong trend in the quantum efficiency as the frequency changes by a factor of ten. The variation in the amount of diffractive loss in the FIR should be large over this range. Furthermore, an optical phonon mode in $\mathrm{LiNbO}_{3}$ at $252 \mathrm{~cm}^{-1}$ would be expected to strongly influence the quantum efficiency through changes in the FIR absorption and phonon resonance enhancement. 12,13

The FIR spectrum (Fig. 28) shows that the FIR was generated in a narrow bandwidth at the frequency difference of the two visible pulses. The FIR bandwidth of $<$ 


\section{0}




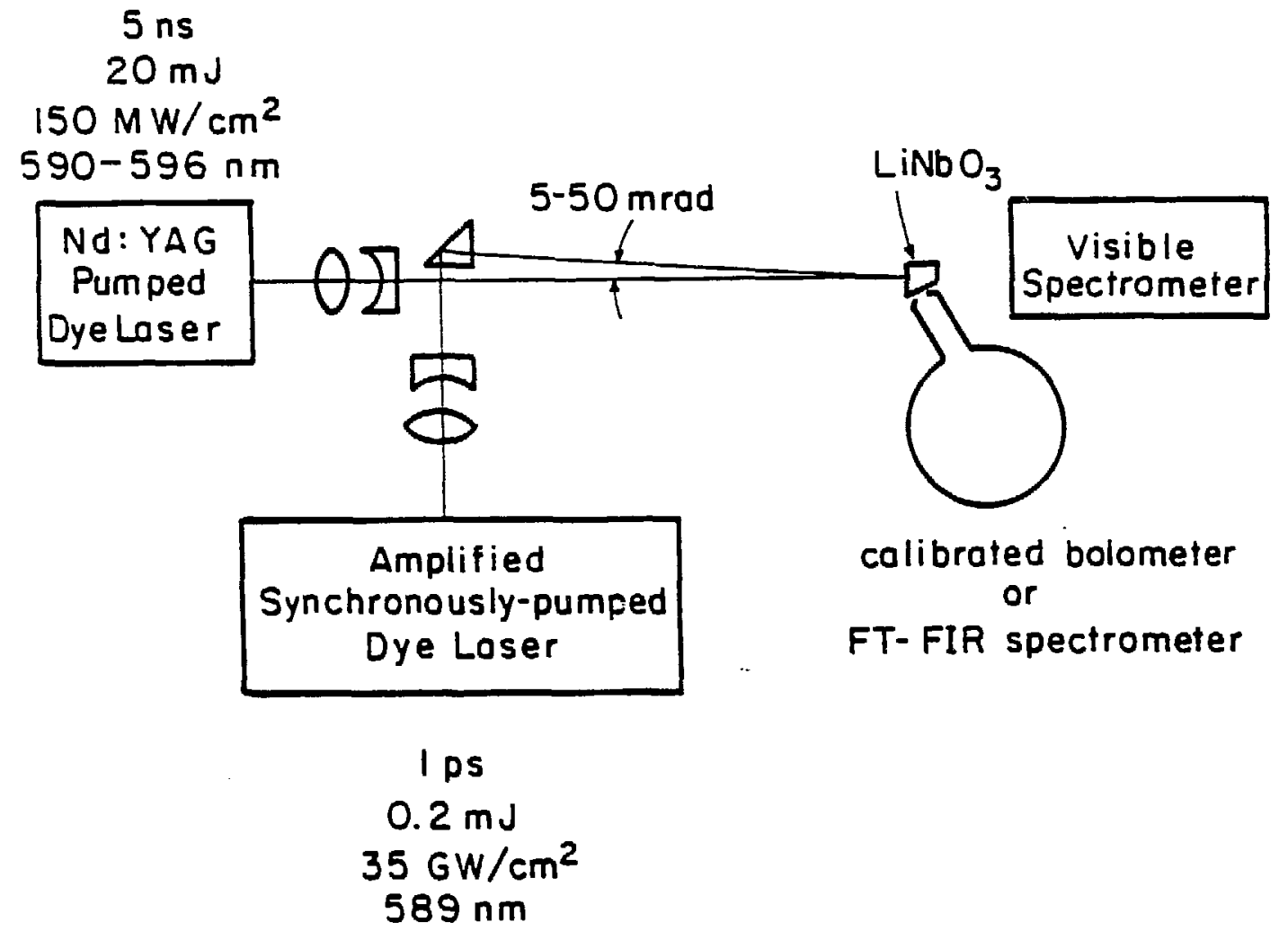

$X B L \quad B 52-1130$

Figure 26 Schematic diagram of the FIR ps pulse apparatus. 

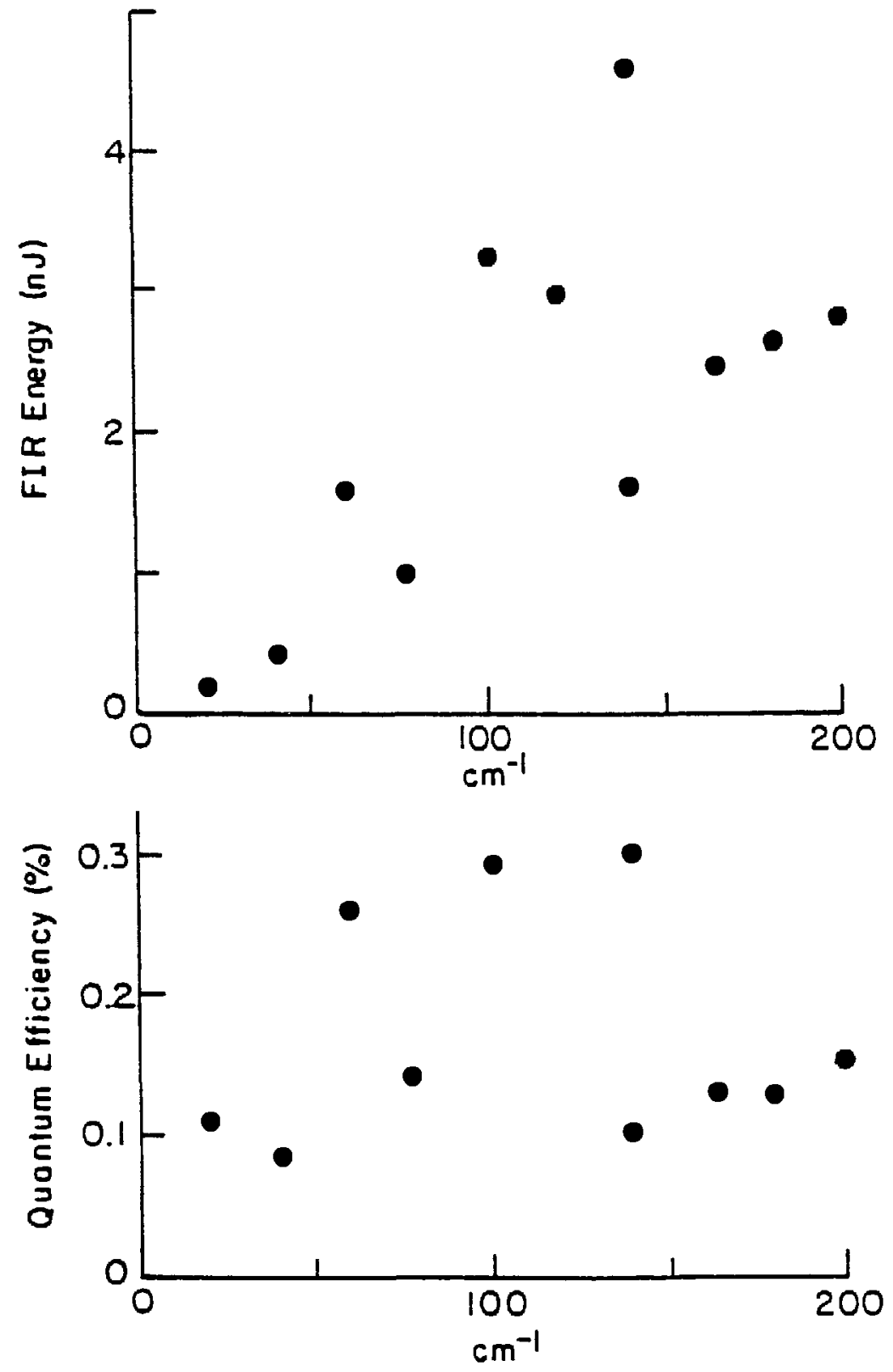

XBL $852-1132$

Figure 27 Total detected energy of the FIR ps pulse as a function of FIR frequency. The quantum efficiency for conversion of the visible to the FIR varies from $0.1 \%$ to $0.3 \%$. 


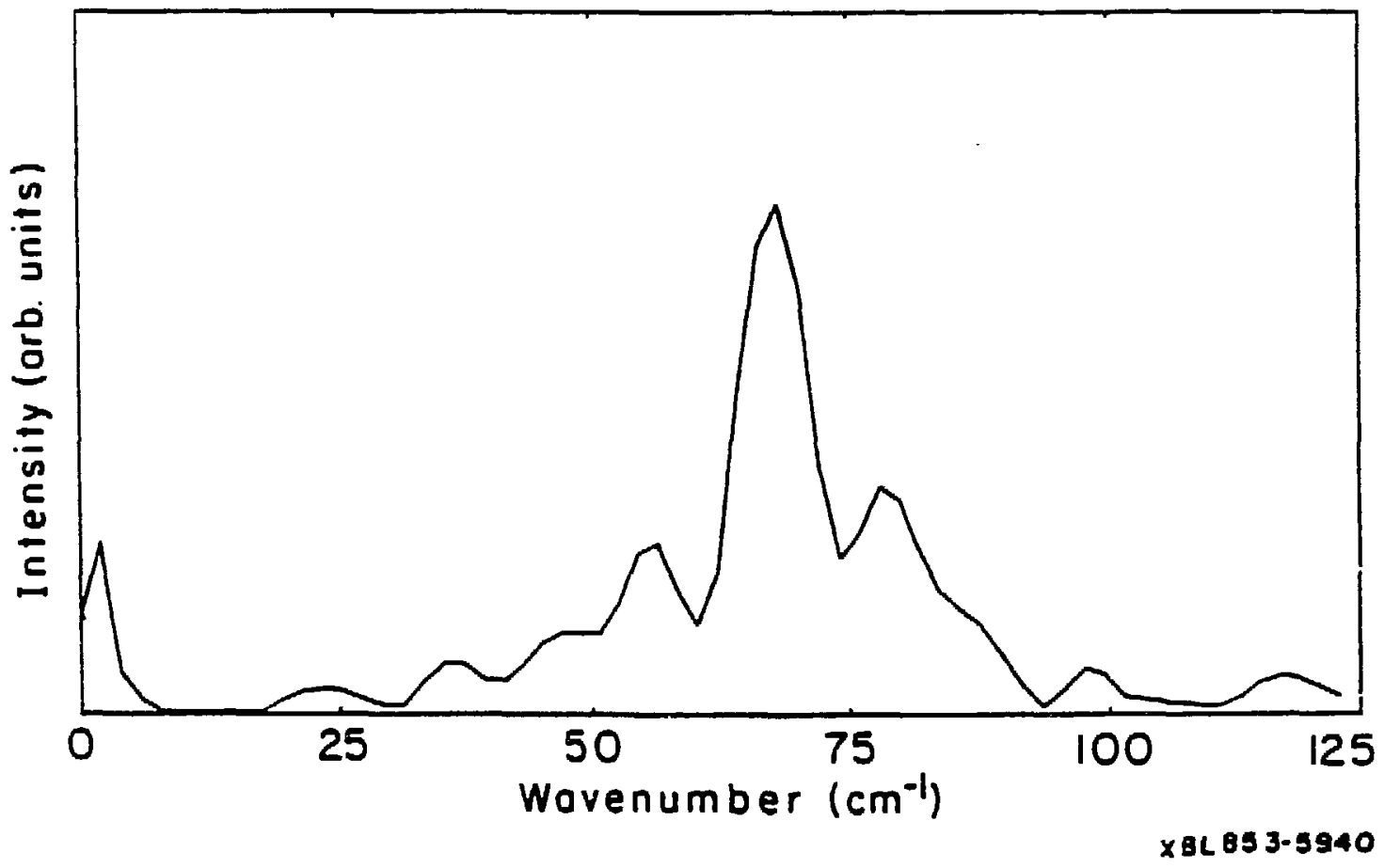

Figure 28 Average spectrum of the FIR generated with the laser difference frequency centered at $70 \mathrm{~cm}^{-1}$. 
$10 \mathrm{~cm}^{-1}$ was similar to the bandwidth of the visible picosecond pulses. Although this bandwidth is consistent with a I ps FIR pulse, consideration of the spatial extent of the region of the crystal that actually radiates FIR suggests that the actual pulse length was closer to $10 \mathrm{ps}$.

The simple theory of parametric mixing predicts that photons from the high frequency picosecond pulse are split into photons at both the lower, nanosecond frequency and into FIR photons. ${ }^{14}$ As long as the nanosecond intensity is low relative to the picosecond intensity, the FIR energy will depend exponentially on the picosecond intensity and linearly on the nanosecond intensity. If the mixing continues until the picosecond pulse is significantly converted, the process will saturate. The FIR energy will then depend linearly on the picosecond energy and will be insensitive to the nanosecond energy.

Experimentally, A small amount of FIR was generated, even when only the picosecond pulse entered the crystal. At the same time, a small amount of visible light was observed to be shifted down in frequency from 0 to $>300 \mathrm{~cm}^{-1}$, but peaked near $150 \mathrm{~cm}^{-1}$ as is shown in Fig. 29. This is interpreted to be parametric fluorescence resulting from the amplification of quantum noise. Optical rectification may also have contributed to the FIR signal. ${ }^{12}$ These observations indicate the strength of the non-linear interactions at the intensities used.

When both the nanosecond and the picosecond pulse were passed through the full 4 $\mathrm{mm}$ length of the crystal, the ficosecond pulse was depleted by $30-50 \%$. With the nanosecond pulse at full intensity, the FIR energy was observed to depend linearly on the picosecond intensity for more than an order of magnitude. The FIR generation appeared to have a threshold near $20 \mu \mathrm{J}$, which may represent the beginning of the sarurated regime. 


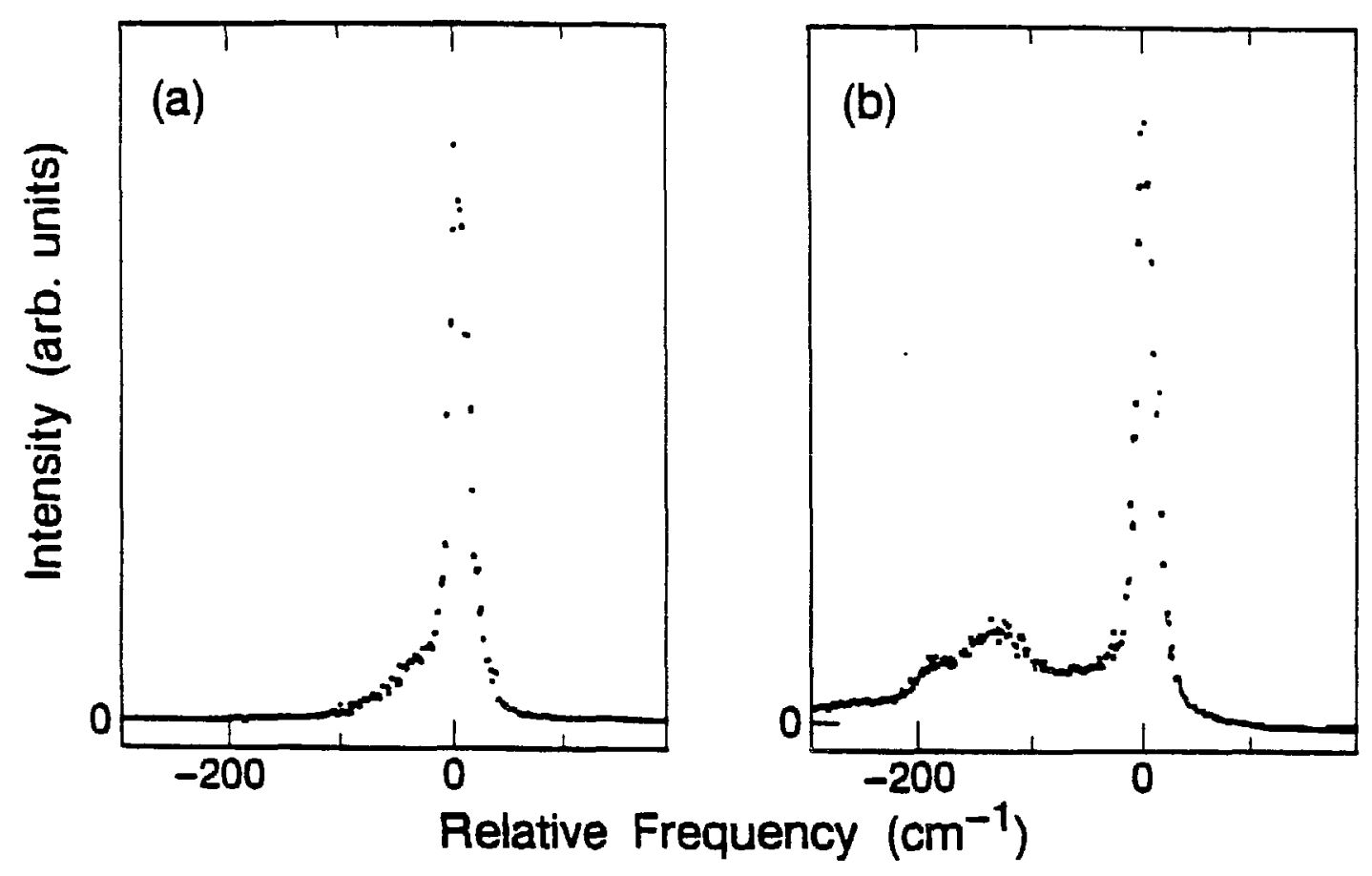

XBL 891.5014

Figure 29 spectra of the optical picosecond pulse before and after passing through the $\mathrm{LiNbO}_{3}$ in the absence of the nanosecond pulse. Significant shifting of the radiation occurs due to oprical rectificarion and noise amplification. 
Finally, the FIR was insensitive to the nanosecond pulse energy; when the nanosecond intensity was reduced by a factor of 7, the FIR intensity only dropped by a factor of 2 and the visible picosecond pulse was still strongly depleted.

\section{Discussion}

All the observations are consistent with a very strong non-linear interaction, leading to strongly saturated FIR generation. However, strongly saturated mixing should lead to high quantum efficiencies. Even considering losses such as reflections at the crystal and FIR absorption by the crystal, the observed quantum efficiencies are surprisingly low. Furthermore, strong pulse-to-pulse fluctuations in FIR energy were observed, whereas saturated mixing should be quite stable. Figure 30 shows a histogram of these fluctuations. All pulses with energy greater than twice the medial energy are shown in the cross-hatched bar at the ight. Some pulses were observed with as much as five times the median pulse energy. However, all pulses generated FIR observable over detector noise. Interestingly, fluctuations of the same magnitude in the depletion of the visible pulse were not observed. Given the observed weak dependence of the FIR on the visible pulse intensities, the $10 \%$ fluctuations of the visible pulse intensities cannot be responsible for the FIR fluctuations. Also, transmission of the beams through a pinhole showed that there is no significant wandering of the beams from pulse to pulse.

Both the low quantum efficiency and the large pulse-to-pulse fluctuations are inconsistent with the simple theory of saturated mixing. Two of the possible explanations for this disagreement are:

1) The FIR is generated efficiently by a saturated process, but is then depleted by further non-linear processes. The flucruations in these processes cause to observed fluctuations in the FIR and are responsible for the discrepancy between depleted 


\section{Pulse Fluctuations}

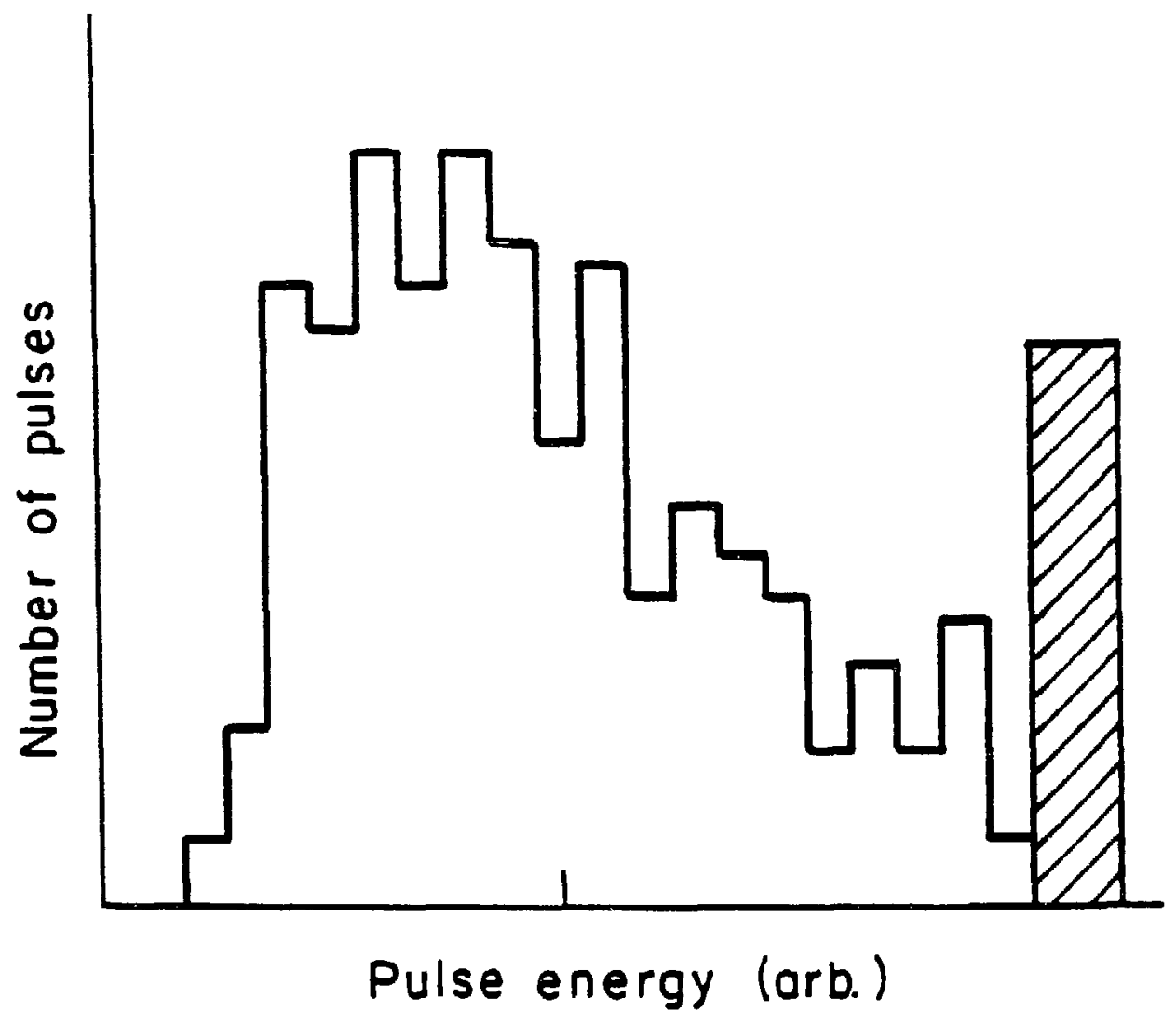

XBL 852-1133

Figure 30 Distribution of the pulse-to -pulse fluctuations of the FIR energy. The cross-hatched region represents all of the pulses with more than twice the median energy. 
picosecond intensity and measured FIR intensity. Hnwever, no reduction in the fluctuations was observed when the beam intensities were reduced.

The nanosecond pulse is not bandwidth-limited, implying that it has significant amplitude modulation. Since the picosecond pulse is not long enough to average these fluctuations, it may see pulse-to-pulse variations that are larger than the total pulse-to-pulse variations. Since the FIR energy is relatively insensitive to the nanosecond energy, these modulations would have to be large.

None of these explanations is entirely satisfactory, but the presence of occasional high efficiency pulses can be seen as encouraging. Their presence implies that a fundamental limit to the conversion efficiency has not yet been reached. If the source of these fluctuations can be understood, it may be possible to consistently generate FIR pulses with the efficiencies which are now seen only erratically. Such pulses, being intense, continuously tunable and narrow bandwidth would be a valuable spectroscopic tool. 


\section{References}

1 K.H. Yang, P.L. Richards, and Y.R. Shen, Appl. Phys. Lett. 19, 320 (1971) ; J.R. Morris and Y.R. Shen, Opt. Commun. 3, 81 (1971).

2 D.H. Auston, K.P. Chung, J.A. Valdmanis, and D.A. Kleinman, Phys. Rev. Lett. 53, 1555 (1984).

3 Since the publication of this article, several FELs have begun to produce FIR pulses, most notably those at UCSB and BNL.

4 For examples see Nonlinear Infrared Generation, Y.R. Shen, ed. (Springer-Verlag, New York, 1977).

5 J.M. Yarborough, S.S. Sussman, H.E. Purhoff, R.H. Pantell, and B.C. Johndon, Appl. Phys. Lett.15, 102 (1969); M.A. Piestrup, R.N. Fleming, and R.H. Pantell, Appl. Phys. Lett. 23, 669 (1975).

${ }^{6}$ K.H. Yang, J.R. Morris, P.L. Richards, and Y.R. Shen, Appl. Phys. Lett. 23, 669 (1973).

7 J.R. Morris, Y.R. Shen, Phys. Rev. A15, 1143 (1977).

8 M.Berg, A.L. Harris, J.K. Brown, and C.B. Harris, Opt. Lett. 9, 50 (1984).

9 I.P. Kaminow and W.D. Johnston, Phys. Rev. 160, 519 (1967).

10 A.S. Barker, Jr., and R. Louden, Phys. Rev. 158, 433 (1967).

IlW.T. Welford and R. Winston, The Optics of Non-imaging Concentrators (Academic Press, Inc., New York, 1978), pp. 47-68.

12 D.R. Bosomvorth, Appl. Phys. Lett. 9, 330 (1966).

13 C.H. Henry and C.G.B. Garrett, Phys. Rev. 171, 1058 (1968). 
14 N. Blcembergen, Non-linear Optics (W.A. Benjarnin, Ins., Reading, Mass., 1965), sec. 4-4. 